FERNANDO DOS SANTOS BARBOSA

\title{
4DOF QUADCOPTER: DEVELOPMENT, MODELING AND CONTROL
}


FERNANDO DOS SANTOS BARBOSA

\title{
4DOF QUADCOPTER: DEVELOPMENT, MODELING AND CONTROL
}

\author{
Dissertation submitted to Escola \\ Politécnica of Universidade de São Paulo \\ for the degree of Master in Science.
}


FERNANDO DOS SANTOS BARBOSA

\section{DOF QUADCOPTER: DEVELOPMENT, MODELING AND CONTROL}

Dissertation submitted to Escola

Politécnica of Universidade de São Paulo for the degree of Master in Science.

Area of Concentration:

Systems Engineering

Advisor:

Prof. Dr. Bruno Augusto Angélico 
Este exemplar foi revisado e corrigido em relação à versão original, sob responsabilidade única do autor e com a anuência de seu orientador.

São Paulo, de de

Assinatura do autor:

Assinatura do orientador:

Catalogação-na-publicação

Barbosa, Fernando dos Santos

4DOF Quadcopter: Development, Modeling and Control / F. S. Barbosa versão corr. -- São Paulo, 2017.

$86 \mathrm{p}$.

Dissertação (Mestrado) - Escola Politécnica da Universidade de São Paulo. Departamento de Engenharia de Telecomunicações e Controle.

1.AERONAVES QUADRIMOTORAS 2.AERONAVES NÃO TRIPULADAS 3.CONTROLE (TEORIA DE SISTEMAS E CONTROLE) I.Universidade de São Paulo. Escola Politécnica. Departamento de Engenharia de Telecomunicações e Controle Il.t. 
To my parents, brother, wife and Spike. 


\section{ACKNOWLEDGMENTS}

First of all, I would like to thank my beloved parents, Fernando Negrão Barbosa and Cassia Regina dos Santos Barbosa, for their endless faith in me and my career and for all the support provided since I was a kid.

I am immensely grateful for being chosen by Nayara Carvalho Tannure to be her eternal partner, and here goes my special thanks to her. Thanks for being supportive, imaginative and patient. I do owe you a lot of what I have conquered.

I cannot forget, either, to thank Prof. Dr. Bruno Augusto Angélico for all the guidance provided during hours and hours of friendly conversations or the guys from Laboratório de Controle Aplicado (LCA) - Gabriel Pereira das Neves, Fabio Yukio Toriumi, Arthur Castello Branco de Oliveira, Mateus, José Genário, Giovanni Gallon Neto - for their friendship and great ideas.

Lastly, thanks to all my family and friends - Felipe, Irineu, Dirce, Manoel, Marilza, aunts, uncles, cousins, Ian, André - for being part of my life. 
"Never discourage anyone... who continually makes progress, no matter how slow." 


\section{RESUMO}

Este texto apresenta o desenvolvimento de um protótipo de quadricóptero com quatro graus de liberdade (4DOF), o qual possibilita a rotação do veículo em torno dos três eixos (yaw, pitch e roll) e o deslocamento ao longo do eixo $z$ (altitude). O objetivo é obter um protótipo de bancada que use a maior quantidade de componentes de um quadricóptero comercial (sensores e atuadores) e usá-lo para a aplicação de controladores de atitude e altitude, utilizando técnicas PID, LQR e Sliding-Mode. Partindo da modelagem do sistema, mostra-se as especificações do mesmo, os componentes utilizados e finaliza-se com o desenvolvimento dos controladores, simulação e aplicação deles.

Palavras-Chave: 4DOF Quadricóptero. Controle de Atitude. Controle de Altitude. LQR. PID. Sliding-Mode. 


\section{ABSTRACT}

This text presents the development of a four-degree-of-freedom (4DOF) quadcopter prototype that allows the vehicle to rotate around the three axes (yaw, pitch and roll) and linear movement along $z$-axis (altitude). The goal is to obtain a prototype bench that uses a good amount of components used in commercial quadcopters (sensors and actuators) and use it to apply attitude and altitude controllers, using techniques such as PID, LQR and Sliding-Mode. Starting from the system modeling, its specifications are shown followed by listing the components used, finishing with the development of the controllers and their simulations and applications.

Keywords: 4DOF Quadcopter. Attitude Control. Altitude Control. LQR. PID. SlidingMode. 


\section{LIST OF FIGURES}

Figure 1 Movement according to motors rotation. . . . . . . . . . . 18

Figure 2 Body and Inertial frames. . . . . . . . . . . . . . . 23

Figure $3 \quad$ Structural components of the prototype. . . . . . . . . . . . 28

Figure 4 Section view of the linear bearings final assembly. . . . . . . . . . . 29

Figure $5 \quad$ CAD image and picture of the 4 DOF solution. . . . . . . . . . . . 29

Figure 6 Propeller guard designed at LCA f . . . . . . . . . . . . . . 30

Figure 7 Bluetooth kit. . . . . . . . . . . . . . . . 30

Figure 8 IMU and distance sensors. . . . . . . . . . . . . . . . . . 31

Figure 9 FreeIMU GUIs. . . . . . . . . . . . . . . . . . . . . 32

Figure $10 \quad$ Schematics of inrunner and outrunner BLDC motors. . . . . . . . . 34

Figure $11 \quad$ ESC, motor, propeller and power supply. . . . . . . . . . . . . . 34

Figure 12 Torque and thrust modes of the BLDC motor test bench. . . . . . . 36

Figure 13 Motor test bench built at LCA . . . . . . . . . . . . . . . . 37

Figure 14 Thrust as function of the squared speed . . . . . . . . . . . . . . 38

Figure 15 Torque as function of the squared speed f . . . . . . . . . . 38

Figure 16 ESC signal percentage as function of the motor speed. . . . . . . . . 39

Figure 17 Step response for the motor/propeller system. . . . . . . . . . . . . 39

Figure 18 ISA form PID block diagram. . . . . . . . . . . . . . . . 41

Figure 19 PID block diagram with output derivative. . . . . . . . . . . . 41

Figure 20 Block diagram of the LQR in regulation form. . . . . . . . . . . . 43

Figure 21 Block diagram of the LQR with integrator insertion. . . . . . . . . . 44

Figure 22 Block diagram of the Sliding Mode controller. . . . . . . . . . . . . 47

Figure 23 Block diagram of the complete system. . . . . . . . . . . . . . 54 
Figure 24 Simulation results for the discretized PID controllers without noise. 58

Figure 25 Simulation results for the PID discretized controllers with noise. . . 59

Figure 26 Practical results for the PID. . . . . . . . . . . . . . . 60

Figure 27 Simulation and test results for the discretized LQR controllers with noise. . . . . . . . . . . . . . . . . . . 62

Figure 28 Controlled states with Sliding Mode Controllers . . . . . . . . . . . 69

Figure 29 Sliding Mode Controllers robustness simulations. . . . . . . . . . . . 69

Figure 30 Simulated and real results with SMC and LQR augmented with integrators. . . . . . . . . . . . . . . . . . 72

Figure 31 Reference tracking simulation results. . . . . . . . . . . . 76

Figure 32 Reference tracking view in 3D. . . . . . . . . . . . . 76

Figure 33 Simulation results for the discretized PID controllers in ISA form. . 84

Figure 34 Sliding Mode Controllers . . . . . . . . . . . . . . . . . . . . . 84

Figure 35 Altitude and $\phi$ response to non-zero initial condition with SMC. . . 85

Figure $36 \quad \theta$ and $\psi$ response to non-zero initial condition with SMC. . . . . . . 86 


\section{LIST OF TABLES}

Table 1 IMU features . . . . . . . . . . . . . . . . . . . . 32

Table 2 Parts weight . . . . . . . . . . . . . 35

Table 3 PID parameters . . . . . . . . . . . . . 56

Table 4 Admissible Parameter Values . . . . . . . . . . . . . . . 63

Table 5 Sliding Mode Controller Parameters . . . . . . . . . . . . . 66

Table 6 Angular Resultant Performance Indices . . . . . . . . . . . . . . . 73

Table $7 \quad$ Altitude Resultant Performance Indices . . . . . . . . . . . . . . . . 73 


\section{LIST OF ABBREVIATIONS}

\begin{tabular}{|c|c|}
\hline BLDC & Brushless DC Motor \\
\hline CAD & Computer-Aided Design \\
\hline DOF & Degrees of Freedom \\
\hline DSLR & Digital Single-Lens Reflex Camera \\
\hline ESC & Electronic Speed Control \\
\hline ETH & Swiss Federal Institute of Technology \\
\hline GRASP & General Robotics, Automation, Sensing, and Perception \\
\hline GUI & Graphical User Interface \\
\hline IAE & Integral Absolute Error \\
\hline IAS & Industry Applications Society \\
\hline IEEE & The Institute of Electrical and Electronics Engineers \\
\hline IMU & Inertial Measurement Unit \\
\hline ISA & International Society of Automation \\
\hline ITAE & Integral Time-weighted Absolute Error \\
\hline LCA & Laboratório de Controle Aplicado \\
\hline LQR & Linear Quadratic Regulator \\
\hline MEMS & Microelectromechanical Systems \\
\hline MIT & Massachusetts Institute of Technology \\
\hline $\mathrm{MPC}$ & Model Predictive Control \\
\hline PID & Proportional-Integrative-Derivative Controller \\
\hline PPM & Pulse-Position Modulation \\
\hline ROS & Robot Operating System \\
\hline UAV & Unmanned Aerial Vehicle \\
\hline VTOL & Vertical Take-Off and Landing Vehicle \\
\hline
\end{tabular}




\section{LIST OF SYMBOLS}

$\mathrm{C}_{\mathrm{H}}$

$\mathrm{G}_{\mathrm{H}}$

$\mathrm{O}_{\mathrm{H}}$

$\Omega$

$\mathrm{U}_{\mathbf{H}}$

$\mathrm{U}_{\mathrm{B}}$

$m$

$\mathbf{I}_{3 \times 3}$

$\mathbf{0}_{3 \times 3}$

I

$I_{x}, I_{y}, I_{z}$

$g$

$J_{T P}$

$T_{j}$

$D_{j}$

$\Omega_{j}$ $b$

$d$
Hybrid UAV vector of reference

Linear position in the $x$-axis of Earth frame

Linear position in the $y$-axis of Earth frame

Linear position in the $z$-axis of Earth frame

Angle rotated around $x$-axis of the Body frame (roll)

Angle rotated around $y$-axis of the Body frame (pitch)

Angle rotated around $z$-axis of the Body frame (yaw)

Mass and inertia matrix in the hybrid reference frame

Coriolis-centripetal matrix in the hybrid reference frame

Gravity vector in the hybrid reference frame

Gyroscopic effect matrix in the hybrid reference frame

Motors rotational speeds vector

Force and torque inputs in the hybrid reference frame

Force and torque inputs in the body reference frame

Mass of the system

Identity matrix of size 3

3-by-3 matrix of zeros

Inertia matrix in the body frame

Inertia around $x, y$ and $z$-axis, respectively

Gravity acceleration

Rotor inertia

$j$-th motor thrust

$j$-th motor drag

$j$-th motor rotational speed

Thrust constant

Drag constant 
R

$c_{\alpha}$

$s_{\alpha}$

$U_{i}$

$K_{c}$

$T_{I}$

$T_{D}$

$e(t)$

$r(t)$

$y(t)$

N

$\mathbf{x}(t)$

$\mathbf{u}(t)$

A, B, C

K

$\mathrm{Q}, \mathbf{R}$

P

$\dot{\mathbf{v}}(t)$

$T_{s}$

$f(\mathbf{x})$

$b(\mathbf{x})$

$\mathbf{x}_{d}$

$\tilde{\mathbf{x}}$

$S(t), s(\mathbf{x}, t)$

$\lambda$

$n$
Body to hybrid frame o reference rotation matrix

Short for cosine of $\alpha$

Short for sine of $\alpha$

Simplified system inputs

Distance between body and motor centers

Sum of the motor speeds

Proportional gain in PID controller

Integrative time in PID controller

Derivative time in PID controller

Error between reference and system output

Reference signal

System output

Low-pass filter parameter

System states

Control signal

State, input and output matrices, respectively

Gain matrix in LQR controller

LQR functional parameters to weight between states error and control effort

Solution to the LQR Riccati equation

LQR integrator output

Sampling time

Nonlinear function not precisely known

Nonlinear function with known sign

Vector of desired system states

Tracking error vector

Sliding surface

Sliding surface parameter

System dimension 
Strictly positive constant

$\hat{f}(\mathbf{x})$

$f(\mathbf{x})$ estimative

F

$\hat{f}(\mathbf{x})$ estimation error

$b_{\min }, b_{\max } \quad b(\mathbf{x})$ minimum and maximum values (bounds)

$\hat{b}(\mathbf{x}) \quad b(\mathbf{x})$ estimative

$k$

Gain to the sliding mode surface sign

$\beta$

$\hat{b}(\mathbf{x})$ estimation error

$\Phi$

Boundary layer

$\mathrm{C}_{\mathrm{o}}$

Controllability matrix

$\mathrm{O}$

Observability matrix 


\section{CONTENTS}

$\begin{array}{lll}1 & \text { Introduction } & 17\end{array}$

1.1 Basic Concepts . . . . . . . . . . . . . . . . 17

1.2 Literature Review . . . . . . . . . . . . . . . . . . . . . . . . . . 19

1.3 Motivation and Objectives ...................... 21

1.4 Published Works . . . . . . . . . . . . . . . . . 21

2 Quadcopter Modeling and Design 23

2.1 UAV Kinematics and Dynamics . . . . . . . . . . . . . . . 23

2.2 Prototype Development . . . . . . . . . . . . . . . 27

2.2 .1 4DOF Solution . . . . . . . . . . . . . . . 27

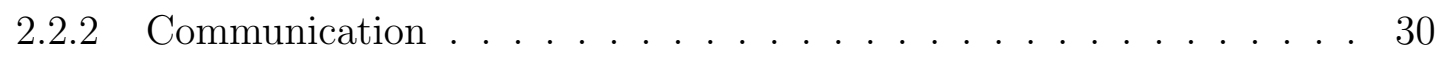

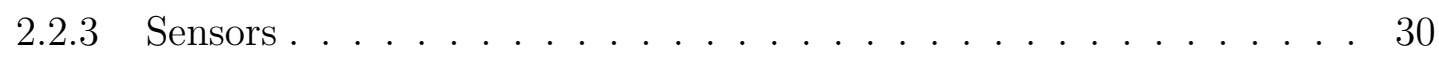

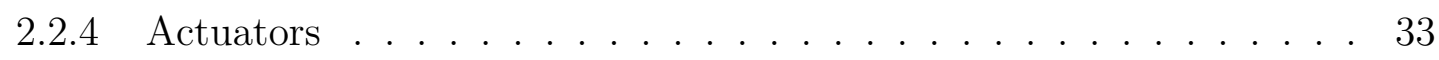

2.2 .5 Arduino Due . . . . . . . . . . . . . . . . 35

2.3 System Parameters . . . . . . . . . . . . . . . . . . . 35

3 Control Techniques $\quad 40$

3.1 PID controller . . . . . . . . . . . . . . . . . 40

3.1.1 Discretization .......................... 42

3.2 LQR Controller . . . . . . . . . . . . . . . . . . . . . . 43

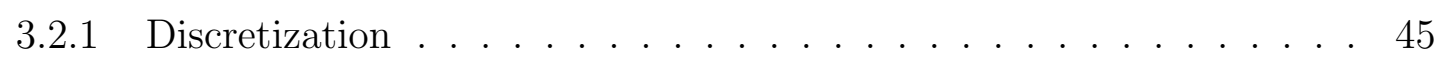

3.3 Sliding Mode Controller . . . . . . . . . . . . . . . . . . 45

3.4 Integrative Sliding Mode Controller with LQR . . . . . . . . . . . . . . 48

3.4 .1 Defining Matrix $\mathbf{G} \ldots \ldots \ldots \ldots$ 
3.5 Performance Indices $\ldots \ldots \ldots \ldots$. . . . . . . . . . . . . . 51

4 Controller Design, Simulation and Results 52

4.1 Simplified Model . . . . . . . . . . . . . . . . . . . . . . 52

4.2 Controllability and Observability . . . . . . . . . . . . . 54

4.3 Tests Procedure . . . . . . . . . . . . . . . . . 55

4.4 PID Controllers . . . . . . . . . . . . . . . . . . 56

4.5 LQR Controller . . . . . . . . . . . . . . . . . . . . . . . . 61

4.6 Sliding Mode Controllers . . . . . . . . . . . . . . . . . . . . . . . . . 62

4.6.1 Altitude Controller . . . . . . . . . . . . . . . . . 63

4.6 .2 Attitude Controllers . . . . . . . . . . . . . . . . . . . 64

4.6.3 Parameters and Specifications . . . . . . . . . . . . . . 66

4.6.4 Reference filtering and its derivatives . . . . . . . . . . . . . 67

4.6.5 Simulation Results . . . . . . . . . . . . . . . . . 67

4.6 .6 Robustness Simulation . . . . . . . . . . . . . . . . 68

4.7 Integrative Sliding Mode Controller with LQR . . . . . . . . . . . . 68

4.8 Resultant Performance Indices . . . . . . . . . . . . . . . . . . . . . 73

4.9 Trajectory Tracking . . . . . . . . . . . . . . . . . . 74

$\begin{array}{llr}5 & \text { Conclusion } & 77\end{array}$

$\begin{array}{lr}\text { References } & 79\end{array}$

Appendix A - Extra Results $\quad 83$

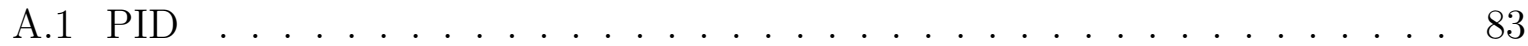

A.2 Sliding Mode . . . . . . . . . . . . . . . . . . . . . 83 


\section{INTRODUCTION}

This chapter is intended to present the basic concepts of a multirotor, the literature review and the works published by the author.

\subsection{Basic Concepts}

Several industries, including automotive, medical, manufacturing and aerospace, require robots to replace humans in dangerous situations (BOUSBAINE; WU; POYI, 2012). Unmanned autonomous vehicles are being developed for in air, land and water use funded by private and government agencies (NICE, 2004). The use of unmanned aerial vehicles, also known by the acronym UAV, is in full expansion throughout the world and is expected to become an important part of the aviation industry in the coming decades (BOUSBAINE; WU; POYI, 2012). They are used for surveillance, disasters exploration (such as fire, earthquake and flood), monitoring leakage of hazardous materials, mobile sensor networks (NEMATI; KUMAR, 2014), agricultural monitoring, onboard image (LEONG; LOW; OOI, 2012), search and rescue, construction and inspection (RAFFO; ORTEGA; RUBIO, 2010), entertainment and military applications of remote surveillance (SANCA, 2013).

Vertical Take-Off and Landing (VTOL) vehicles (MARTINEZ, 2007) such as conventional helicopters and multirotors (multiple rotors vehicles) benefit from good mobility, simple mechanism and higher load capacity (MUSTAPA et al., 2014). Its civilian use versions appeared as they are attractive for surveillance, photographing and filming hazardous environments and external navigation and mapping (NEMATI; KUMAR, 2014). Although the use of large UAVs nowadays is becoming more usual, the efforts made to use this technology on a smaller scale are few (BEFINS, 2014).

The best known multirotors are tricopters (trirotors), quadcopters (quadrotors) and hexacopters (hexarotors), respectively having 3,4 and 6 sets of motors and propellers (SANCA, 2013). Historically, four-rotor helicopters were out of the ordinary, primarily 
because most loads could be lifted by one or two rotors (MARTINEZ, 2007). The very first quadcopter was built in the 1920s and carried passengers (SA; CORKE, 2011); in spite of the progress, building a completely autonomous one is still a challenge (MARTINEZ, 2007). Nevertheless, its simplicity makes it easy and cheap to build, making possible to find low-cost radio controlled toys (SA; CORKE, 2011) and flight controller boards, such as KKmulticopter ${ }^{\circledR}$ and DJI Naza ${ }^{\circledR}$ (LEONG; LOW; OOI, 2012).

Due to the advancement in microelectromechanical systems (MEMS), electric energy accumulators, actuators, and the reduction in circuit scale integration, the number of studies on UAVs has increased not only in universities but also in institutions for use in civil and military applications (OLIVEIRA, 2011), and has generated great interest in the automatic control area over the last decades (RAFFO; ORTEGA; RUBIO, 2010).

Multirotors provided VTOLs with simplicity since, as compared to usual helicopters, they present less mechanical, electrical and control complexity. The main concept of single and double-rotor helicopters is to keep constant propeller speed and change its pitch in order to control and to stabilize the vehicle, a process that requires precise mechanical structure, sensors and control techniques. On the other hand, the desired maneuvers of multirotors are achieved by simply varying the rotation speed of the motors, as they have fixed pitch propellers (SANCA, 2013).

A quadrotor is a vehicle equipped with four rotors symmetrically disposed around its center (MARTINEZ, 2007). All the motors are admitted to be equal in term of force and torque generation. Vehicle roll, pitch and yaw movements are determined by each motor speed (MUSTAPA et al., 2014), with two motors rotating clockwise and two counterclockwise, alternately, as shown in Figure 1, for balancing the angular moment created by each rotor (NEMATI; KUMAR, 2014). A quadrotor is a multivariable, underactuated, strongly coupled (SANCA, 2013; MIAN; DAOBO, 2008), highly nonlinear system with variable behavior over time, subjected to non-modeled dynamics and parametric uncertainties (RAFFO; ORTEGA; RUBIO, 2010).

Figure 1: Movement according to motors rotation. Thicker arrows show higher speeds.

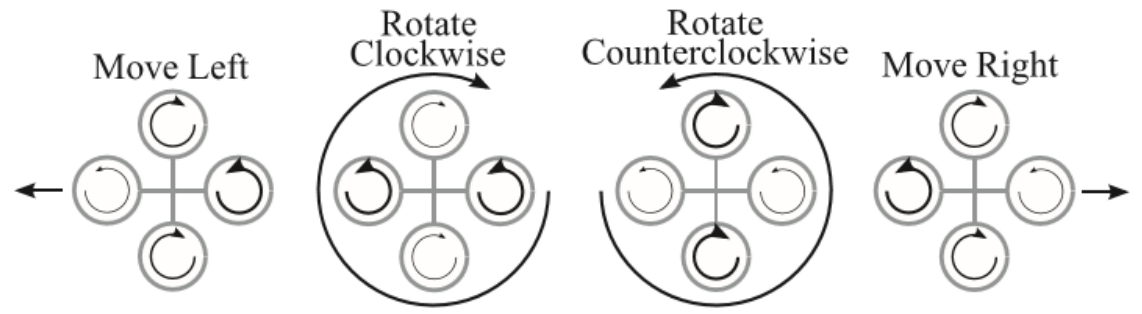

Source: Nicol, Macnab and Ramirez-Serrano (2011) 


\subsection{Literature Review}

Multirotors have a wide research application throughout the world, with themes that include modeling, control, collision avoidance, path tracking, learning mechanisms and cooperative work.

Numerous masters and $\mathrm{PhD}$ works study the modeling process of a quadcopter. Bresciani (2008) uses the Newton-Euler formalism to obtain the dynamic equations, with sufficient attention to the rotor system, composed of DC-motor, gear box and propeller. Nowadays, brushed DC-motors are outdated for this application, and Brushless DC (BLDC) motors are used instead, as shows Pfeifer (2013), who models its dynamics by a simple first-order system. Luukkonen (2011) compares the use of Newton-Euler and EulerLagrange equation to derive the system equations, while Bousbaine, Wu and Poyi (2012) proposes a simulation environment.

Several types of controllers have been implemented. The linear ones are the most usual, among which the Linear Quadratic Regulator (LQR) is compared to the Pole Placement technique by Pfeifer (2013) and to the PID by Bouabdallah (2007), Hystad (2015), Bresciani (2008), Leong, Low and Ooi (2012) and Argentim et al. (2013). The PID controller is not only used to stabilize the attitude as Nice (2004) does, but also to control the altitude, as presented in Mustapa et al. (2014) and Luukkonen (2011); the latter neglects the integral term.

Raffo, Ortega and Rubio (2010) propose a nonlinear $\mathrm{H}_{\infty}$ controller to stabilize the rotational movements and a Model Predictive Controller (MPC) to track the trajectory. Huynh, Zhao and Xie (2014) deal with wind and change of mass with an $\mathrm{L}_{1}$ Adaptive Controller, guaranteeing robustness for outdoors flights. Mian and Daobo (2008) consider the use of two controllers: a feedback linearization with a PD controller for the translational subsystem and a backstepping-based PID controller for the rotational one. Sliding mode based controllers have also been studied, such as in Xiong and Zheng (2014) and Zheng, Xiong and Luo (2014): the former proposes a Terminal Sliding Mode Control and the latter a Second Order one. It has also been used for trajectory tracking with a nonlinear sliding surface as in Sumantri et al. (2013).

Euler angles are the most widely used to represent the angles of the system and to provide references to the controllers. However, based on the fact that the sensors filtering already uses quaternions representation to prevent gimbal locking, a novel controller design based on quaternions has been proposed and promises to be more computationally 
efficient, since the results of the filters do not have to be translated into Euler angles. Tayebi and McGilvray (2006) use a $\mathrm{PD}^{2}$ feedback structure, where the proportional action is in terms of the vector quaternion and the two derivative actions are in terms of the airframe angular velocity and the vector quaternion velocity. Both Cariño, Abaunza and Castillo (2015) and Fresk and Nikolakopoulos (2013) present the quaternion based modeling process of a quadcopter and apply different controllers: the former proposes one controller to stabilize the vehicle heading and one to control its position, while the latter controls the attitude with a nonlinear Proportional Squared $\left(\mathrm{P}^{2}\right)$ control algorithm.

In the robotics field, there is always an interest in creating an intelligent system capable of learning from its experience. Hehn and D'Andrea (2014) apply a learning algorithm carried out in frequency domain, based on a Fourier series decomposition of the input and output signals, to improve the quadcopter behavior for periodic flight maneuvers; Figueroa et al. (2014) use the Reinforcement Learning algorithm to balance an inverted pendulum on a UAV; Lupashin and D'Andrea (2011) create an Adaptive OpenLoop algorithm to perform and to improve aerobatic maneuvers; Ritz and D'Andrea (2014) propose an on-board computationally lightweight iterative learning scheme for improving the performance of highly dynamic open-loop maneuvers.

Three of the main multirotor testbeds built in the world are at ETH Zurich, MIT and University of Pennsylvania. The one in Zurich is called "The Flying Machine Arena" and its construction is detailed in Lupashin et al. (2014). This arena has been used for several works, such as in Hehn and D'Andrea (2011a) for balancing an inverted pendulum with a quadcopter, in Mueller and D'Andrea (2014) for maintaining an altitude despite the complete loss of one, two or three propellers, in Mueller, Hamer and D'Andrea (2015) for fusing Ultra-Wideband (UWB) ranging radios with an IMU for state estimation and in Hehn and D'Andrea (2011b) for trajectory generation and control. Valenti et al. (2006) present the MIT testbed, which mixes aerial and ground vehicles to study long duration missions in a controlled environment. Lastly, Michael et al. (2010) provide the description of the GRASP testbed, used to support research on coordinated dynamic flight, as well as basic modeling and control of attitude, position and 3D trajectory. A stationary quadcopter test bench is proposed by Yu and Ding (2012) and consists of a vehicle attached to a sphere joint (3DOF rotational movement) and to a $6 \mathrm{DOF}$ force/torque sensor.

A very complete tutorial about multirotor aerial vehicles was published by Mahony, Kumar and Corke (2012) and describes not only basic concepts, such as modeling, estimation and control, but also describes the size, weight and power (SWAP) constraints and scaling laws, blade flapping and induced drag. 


\subsection{Motivation and Objectives}

Unmanned Aerial Vehicles have been the center of several studies throughout the world and will probably be for many other years. There are many published applications that still rely on simulations only and need to be implemented. Therefore, a first motivation for this work is to better understand the system and its challenges.

Studying attitude and altitude controllers for quadcopters is not the great focus of most leading laboratories anymore, but it was some years ago, when they started. As our laboratory (Laboratório de Controle Aplicado - LCA) has just started researching this field, this theme is fundamental.

A test bench to which the quadcopter keeps attached all the time is proposed seeing that we do not have enough room to create an indoor test bed. The proposed test bench will provide the basic motions of a UAV - 3 axis rotation and altitude movement - and will be safe and impact-free.

This Master's thesis comprehends a complete engineering project and involves technological and scientific aspects. From a technological point of view, it requires components specifications, mechanical design and prototype assembly. Additionally, the mathematical modeling and controllers design, both meet performance and robustness requirements.

The main objectives of this work are:

- to build a safe and functional 4-degree-of-freedom quadcopter test-bench;

- to develop and test different attitude and altitude controllers.

\subsection{Published Works}

During the development of this Master's Thesis, some works were published in partnership with other members of the laboratory studying the application of different controllers to a 2-DOF Helicopter, a Quadruple Tank system and a Control Moment Gyroscope. Three were published in international IEEE conferences, one in a Brazilian conference and one in a journal, all listed below:

- AngÉlico, BRUnO A. ; BARBOSA, FERnANDO S. ; TORIUMi, FABIO Y.. State Feedback Decoupling Control of a Control Moment Gyroscope. Journal of Control, Automation and Electrical Systems, 2016; 
- BARBosa, F. S.; NEVES, G. P. ; ANGÉLICO, BRUNO A. . Discrete LQG/LTR Control Augmented by Integrators Applied to a 2-DOF Helicopter. In: IEEE MultiConference on Systems and Control 2016, 2016, Buenos Aires. MSC 2016, 2016;

- Neves, G. P. ; BARbosa, F. S. ; COSTA, P. S. ; AngÉlico, BRUno A. . Discrete Time LQG/LTR Applied To A Practical Quadruple Tank System. In: IEEE Multi-Conference on Systems and Control 2016, 2016, Buenos Aires. MSC 2016, 2016;

- Gallon Neto, G.; BARBOSA, F. S. ; ANGÉLICO, BRUNO A.. 2-DOF Helicopter Controlling by Pole-Placements. In: 12th IEEE/IAS International Conference on Industry Applications, 2016. Induscon 2016;

- Barbosa, F. S.; Gallon neto, G. ; AngÉlico, BrUno A. . Digital Integrative LQR Control of a 2DOF Helicopter. In: XXI Congresso Brasileiro de Automática, 2016, Vitória. CBA 2016, 2016. 


\section{QUADCOPTER MODELING AND DESIGN}

The first part of this chapter shows the complete modeling of a quadcopter. Then, the mechanical solution for building a four degree-of-freedom system is presented along with its sensors and hardware. Lastly, the required parameters are identified.

\subsection{UAV Kinematics and Dynamics}

Kinematics studies the movement of a body without considering its causes, i.e., it studies the bodies motion regardless of the forces and torques responsible for it. On the other hand, dynamics studies the effects of forces and torques applied to bodies.

Two reference frames are used for the modeling process: an earth-fixed and a bodyfixed, as shown in Figure 2. This figure also presents the direction of rotation of each motor.

Figure 2: Quadrotor configuration frame system with a body fixed frame B and the inertial frame E. The circular arrows indicate the direction of rotation of each propeller.

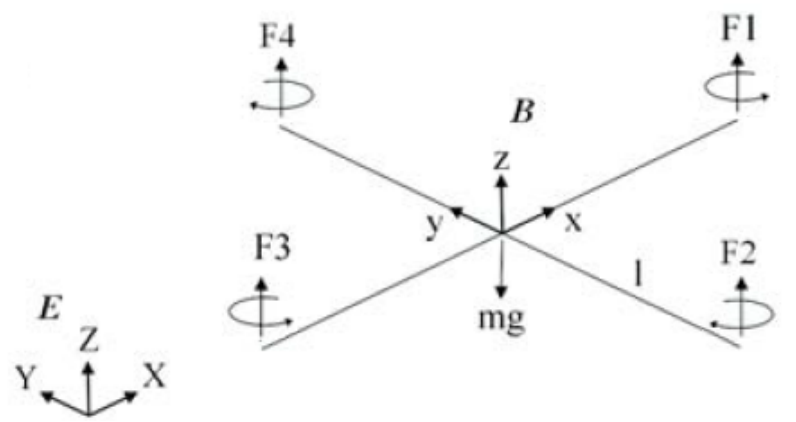

Source: Bouabdallah (2007)

Note that using the UAV as a reference turns all the modeling process easier due to:

- Time-invariant inertia matrix;

- The quadrotor symmetry simplifies the equations; 
- On-board sensors on the same reference.

Moreover, the following must be assumed:

- The body center of mass is coincident with the origin of the coordinate system;

- UAV axis are coincident with the main inertia axis and the inertia matrix is thus diagonal.

Since the interest is to control the angles of the UAV and its localization in space, a hybrid vector is used to describe the system (BRESCIANI, 2008; BOUABDALLAH, 2007):

$$
\boldsymbol{\zeta}=\left[\begin{array}{llllll}
\dot{X} & \dot{Y} & \dot{Z} & p & q & r
\end{array}\right]^{T}
$$

where the first three components represent the linear speeds in the earth frame and the last three the angular velocities in the body frame, which are related to the roll-pitch-yaw set of Euler angles by

$$
\left[\begin{array}{c}
\dot{\phi} \\
\dot{\theta} \\
\dot{\psi}
\end{array}\right]=\left[\begin{array}{ccc}
1 & s_{\phi} t_{\theta} & c_{\phi} t_{\theta} \\
0 & c_{\phi} & -s_{\phi} \\
0 & s_{\phi} / c_{\theta} & c_{\phi} / c_{\theta}
\end{array}\right]\left[\begin{array}{l}
p \\
q \\
r
\end{array}\right],
$$

with $c_{\alpha}$ short for $\cos \alpha, s_{\alpha}$ for $\sin \alpha$ and $t_{\alpha}$ for $\tan \alpha$.

Using Newton-Euler equations, the system dynamics with reference to the hybrid vector can be written as

$$
\mathrm{M}_{\mathbf{H}} \dot{\boldsymbol{\zeta}}+\mathrm{C}_{\mathbf{H}}(\zeta) \zeta=\mathrm{G}_{\mathbf{H}}+\mathrm{O}_{\mathbf{H}}(\zeta) \Omega+\mathrm{U}_{\mathbf{H}}
$$

in which the left part represents the internal dynamics while the right part represents the external forces applied to the body. $\mathbf{M}_{\mathbf{H}}$ is the following inertia matrix

$$
\mathbf{M}_{\mathbf{H}}=\left[\begin{array}{cc}
m \mathbf{I}_{3 \times 3} & \mathbf{0}_{3 \times 3} \\
\mathbf{0}_{3 \times 3} & \mathbf{I}
\end{array}\right]=\operatorname{diag}\left(m, m, m, I_{x}, I_{y}, I_{z}\right),
$$

which is valid since the body-fixed frame is coincident with the system center of mass and aligned with the main axes of inertia, being $m$ the total mass and $I_{x}, I_{y}$ and $I_{z}$ the inertia 
with relation to the body-fixed frame. $\mathbf{C}_{\mathbf{H}}$ is the Coriolis-centripetal matrix, defined as

$$
\mathbf{C}_{\mathbf{H}}(\boldsymbol{\zeta})=\left[\begin{array}{cccccc}
0 & 0 & 0 & 0 & 0 & 0 \\
0 & 0 & 0 & 0 & 0 & 0 \\
0 & 0 & 0 & 0 & 0 & 0 \\
0 & 0 & 0 & 0 & I_{z} r & -I_{y} q \\
0 & 0 & 0 & -I_{z} r & 0 & I_{x} p \\
0 & 0 & 0 & I_{y} q & -I_{x} p & 0
\end{array}\right] .
$$

The gravity $(g)$ influence is represented as $\mathbf{G}_{\mathbf{H}}$ and simply defined as

$$
\mathbf{G}_{\mathbf{H}}=\left[\begin{array}{llllll}
0 & 0 & -m g & 0 & 0 & 0
\end{array}\right]^{T}
$$

since it only affects the Z-axis of the earth frame. The gyroscopic effect caused by the propellers is taken into account in the $\mathbf{O}_{\mathbf{H}}(\boldsymbol{\zeta}) \boldsymbol{\Omega}$, and it shows a crossed influence between $\dot{\phi}$ and $\dot{\theta}$ :

$$
\mathbf{O}_{\mathbf{H}}(\boldsymbol{\zeta}) \boldsymbol{\Omega}=J_{T P}\left[\begin{array}{cccc}
0 & 0 & 0 & 0 \\
0 & 0 & 0 & 0 \\
0 & 0 & 0 & 0 \\
q & -q & q & -q \\
-p & p & -p & p \\
0 & 0 & 0 & 0
\end{array}\right] \boldsymbol{\Omega}
$$

where $J_{T P}$ is the rotor inertia and $\Omega$ is the vector of the motors rotational speeds

$$
\boldsymbol{\Omega}=\left[\begin{array}{llll}
\Omega_{1} & \Omega_{2} & \Omega_{3} & \Omega_{4}
\end{array}\right]^{T}
$$

The last element of the system equation represents the force and torques produced by the rotors which, presented in relation to the body frame, may be written as

$$
\mathbf{U}_{\mathbf{B}}=\left[\begin{array}{llllll}
0 & 0 & \sum_{j=1}^{4} T_{j} & l\left(T_{4}-T_{2}\right) & l\left(T_{3}-T_{1}\right) & \sum_{j=1}^{4}(-1)^{j} D_{j}
\end{array}\right]^{T}
$$

where $T_{j}$ is the $j$-th motor thrust and $D_{j}$ the $j$-th motor drag. As stated in Bresciani (2008), these parameters are both proportional to the squared motor rotation, i.e.,

$$
T_{j}=b \Omega_{j}^{2} \quad \text { and } \quad D_{j}=d \Omega_{j}^{2}
$$

with $b$ and $d$ assumed equal to all motors. Writing equation (2.9) in the hybrid frame 
requires the use of the rotation matrix $\mathbf{R}$, given by

$$
\mathbf{R}=\left[\begin{array}{ccc}
c_{\psi} c_{\theta} & -s_{\psi} c_{\phi}+c_{\psi} s_{\theta} s_{\phi} & s_{\psi} s_{\phi}+c_{\psi} s_{\theta} c_{\phi} \\
s_{\psi} c_{\theta} & c_{\psi} c_{\phi}+s_{\psi} s_{\theta} s_{\phi} & -c_{\psi} s_{\phi}+s_{\psi} s_{\theta} c_{\phi} \\
-s_{\theta} & c_{\theta} s_{\phi} & c_{\theta} c_{\phi}
\end{array}\right]
$$

Therefore, the vector of force and torques produced by the motors can be written in the hybrid frame as

$$
\mathbf{U}_{\mathbf{H}}=\left[\begin{array}{cc}
\mathbf{R} & \mathbf{0}_{3 \times 3} \\
\mathbf{0}_{3 \times 3} & \mathbf{I}_{3 \times 3}
\end{array}\right] \mathbf{U}_{\mathbf{B}}=\left[\begin{array}{c}
\left(s_{\psi} s_{\phi}+c_{\psi} s_{\theta} c_{\phi}\right) U_{1} \\
\left(-c_{\psi} s_{\phi}+s_{\psi} s_{\theta} c_{\phi}\right) U_{1} \\
\left(c_{\theta} c_{\phi}\right) U_{1} \\
U_{2} \\
U_{3} \\
U_{4}
\end{array}\right],
$$

with

$$
\left[\begin{array}{l}
U_{1} \\
U_{2} \\
U_{3} \\
U_{4}
\end{array}\right]=\left[\begin{array}{c}
b\left(\Omega_{1}^{2}+\Omega_{2}^{2}+\Omega_{3}^{2}+\Omega_{4}^{2}\right) \\
l b\left(\Omega_{4}^{2}-\Omega_{2}^{2}\right) \\
l b\left(\Omega_{3}^{2}-\Omega_{1}^{2}\right) \\
d\left(-\Omega_{1}^{2}+\Omega_{2}^{2}-\Omega_{3}^{2}+\Omega_{4}^{2}\right)
\end{array}\right] .
$$

Finally, putting all these matrices together, the system equations are:

$$
\begin{aligned}
\ddot{X} & =(\sin \psi \sin \phi+\cos \psi \sin \theta \cos \phi) \frac{U_{1}}{m} \\
\ddot{Y} & =(-\cos \psi \sin \phi+\sin \psi \sin \theta \cos \phi) \frac{U_{1}}{m} \\
\ddot{Z} & =-g+(\cos \theta \cos \phi) \frac{U_{1}}{m} \\
\dot{p} & =\frac{I_{y}-I_{z}}{I_{x}} q r-\frac{J_{T P}}{I_{x}} q \Omega+\frac{U_{2}}{I_{x}} \\
\dot{q} & =\frac{I_{z}-I_{x}}{I_{y}} p r+\frac{J_{T P}}{I_{y}} p \Omega+\frac{U_{3}}{I_{y}} \\
\dot{r} & =\frac{I_{x}-I_{y}}{I_{z}} p q+\frac{U_{4}}{I_{z}}
\end{aligned}
$$

with $\Omega=-\Omega_{1}+\Omega_{2}-\Omega_{3}+\Omega_{4}$. 


\section{$2.2 \quad$ Prototype Development}

This subsection provides the description of the prototype and what it uses to make the 4DOF movement possible. Then, sensoring and actuators are also described.

\subsubsection{DOF Solution}

The first step in the development of any prototype is to determine its desired characteristics and requirements. In this project, the following should be met:

- being small so as not to disturb or to occupy much space;

- consuming power that still allows the use of low-cost $12 \mathrm{~V}$ power supply;

- minimizing the all-up weight;

- minimizing the air cushion beneath it;

- allowing yaw, pitch and roll rotation and height displacement.

These requirements led us to choose the 250mm Racing Quadcopters category: small yet powerful setup that allows an all-up weight of $1 \mathrm{~kg}$. The access to the components used in this setup was facilitated due to its fame among hobbyists, which made companies create and sell targeted products and ready-to-use kits. Figure 3a presents the frame used in this project.

To solve the four degrees of freedom movement problem, the solution was separated into three-axis rotation and one-axis displacement. An easy way of solving the former is using a sphere joint, a commonly used mechanical component. However, it is neither easy nor cheap to manufacture one. The solution was to search for a small and light sphere joint, and it was found that they are used in DSLR (Digital Single-Lens Reflex) cameras tripods and sold in several sizes. The one chosen, shown in Figure 3b, is rated to hold up to $2 \mathrm{~kg}$.

A low-weight solution that allows z-axis movement is fixing the UAV to a shaft and let it slide through a linear bearing. Not making the quadcopter itself slide through the shaft guarantees that the sensors can still be installed in the center of mass. One should keep in mind that even well manufactured bearings allow the shaft to slightly tilt inside it. Hence, trying to minimize it, two of the LM6UU linear bearing shown in Figure 3c were chosen to be used with a 60-centimeter-long shaft. 
To prevent the quadcopter from suffering ground effect, the first idea was to use PVC pipes to create a high structure such as the one shown in Figure $3 \mathrm{~d}$, in which the linear bearings would be fixed to the vertical part and the shaft would slide inside it. Although it seemed to be a good solution, PVC pipe connections may become loose and the material itself is not stiff enough.

Figure 3: Structural components of the prototype.

(a) $250 \mathrm{~mm}$ quadcopter frame.

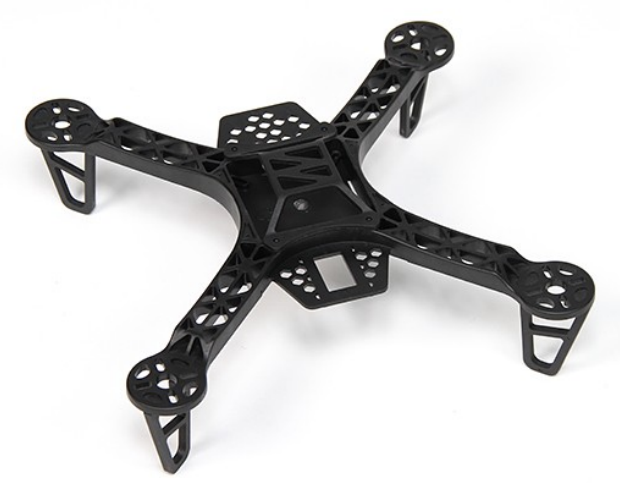

(b) DSLR camera ball head.

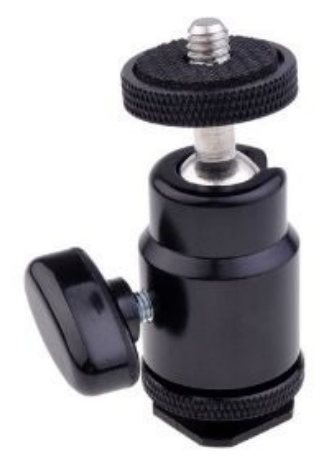

(c) LM6UU linear bearing.

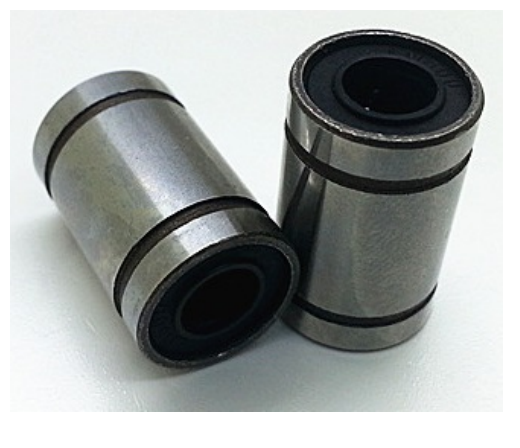

(d) First version of the structure.

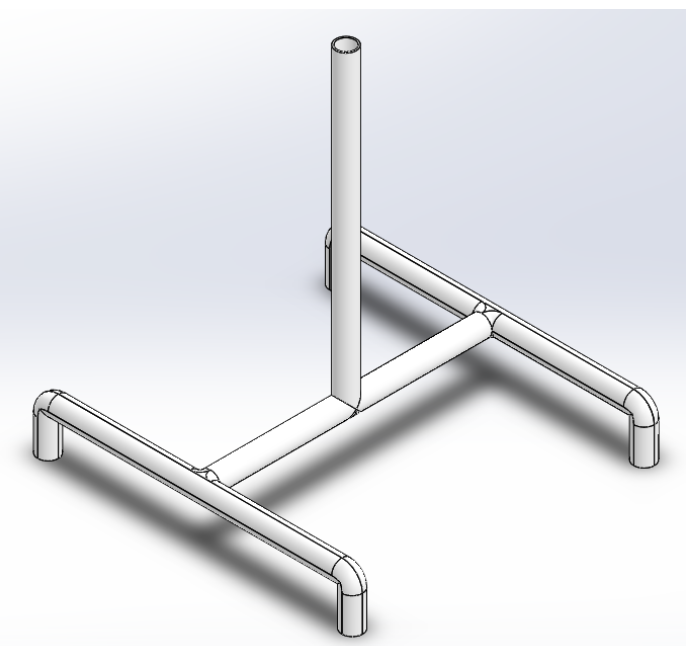

Source: (a) www.hobbyking.com, (b)(c) www.mercadolivre.com.br, (d) author

A wood billet with a hole along it, just like a wood pipe, was used to solve this problem, since it is far more resistant and stiffer than the PVC one. As the PVC structure was already made, the bearings were already fixed on and could not be removed without damage. Thus, the vertical PVC pipe was inserted inside the wood billet and the bearings were glued to an aluminum jacket, as in Figure 4.

Figure 5 presents another view of the final assembly, with the CAD design on the left and a picture of the assembled prototype on the right. The black component is the frame; 
holding it is the DSLR camera ball head, which is fixed to a shaft that slides through the linear bearings attached to the wood billet. This is what we named "4DOF solution".

Figure 4: Section view of the linear bearings final assembly.

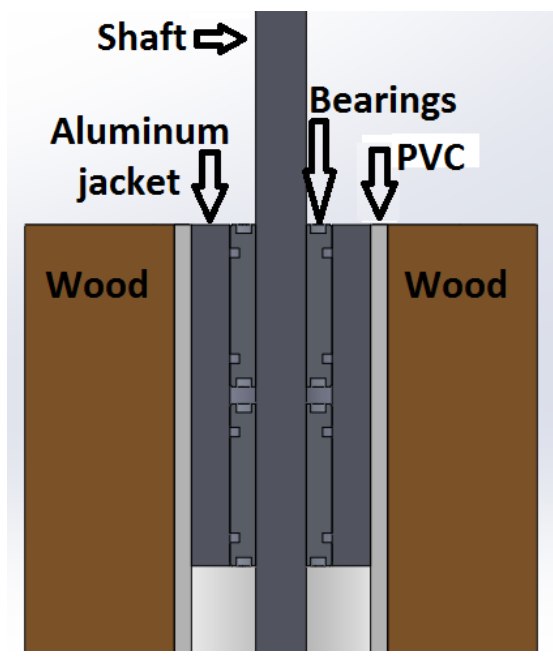

Source: author

Figure 5: CAD image and picture of the 4DOF solution.
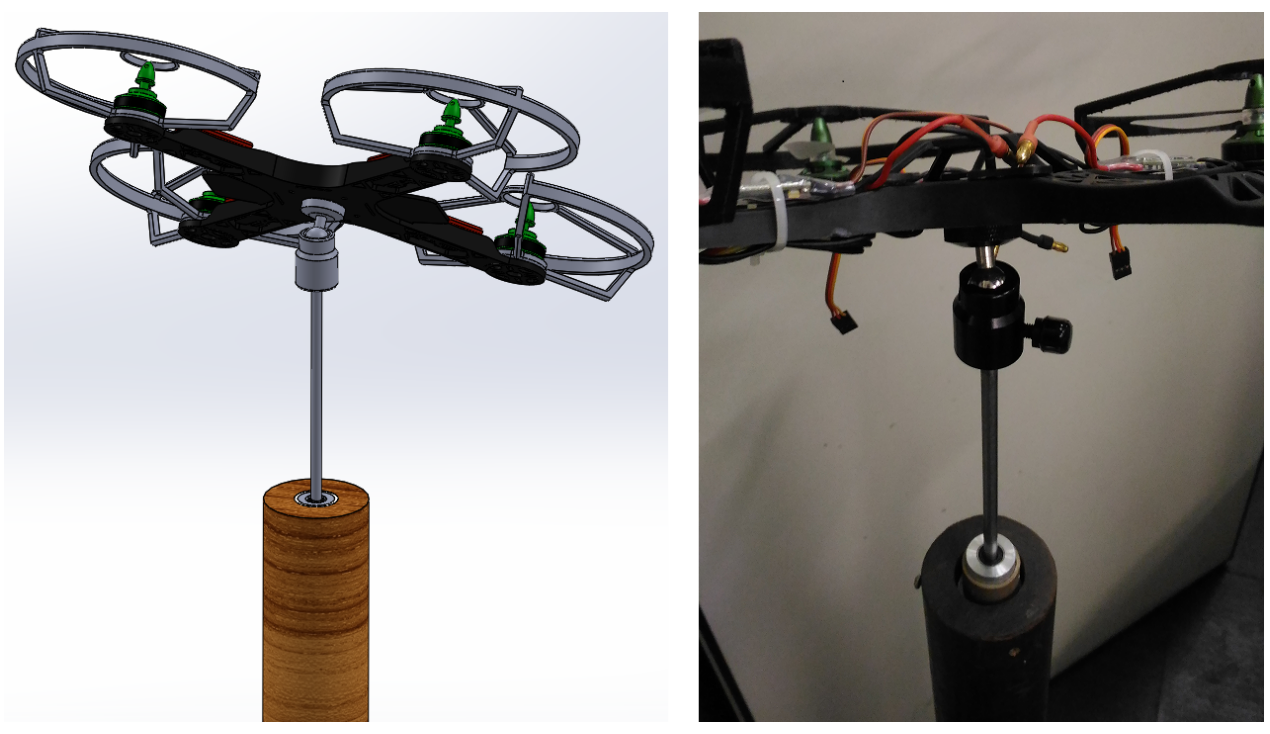

Source: author

The last component to be described here is the propeller guard, designed for protection, mainly. Even though its name suggests that it protects the propeller, it was designed to protect anyone who is using the prototype or is around it. Even plastic propellers act as knives when rotating at over 20,000RPM. Since no item was found for sale that offers reliable protection, one was designed and 3D printed at LCA, which is presented in Figure 6 . 
Figure 6: Propeller guard designed at LCA on the left and 3D printed on the right.
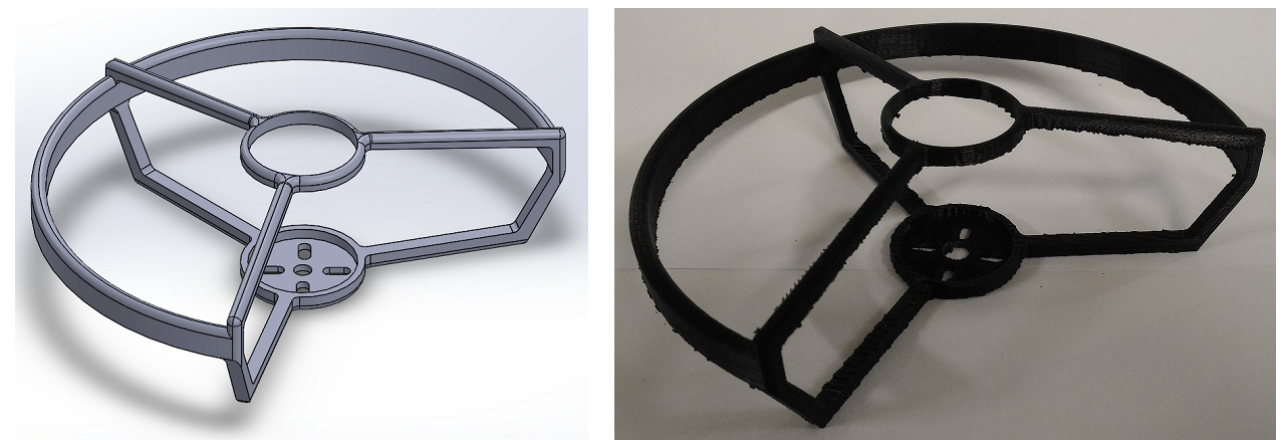

Source: author

\subsubsection{Communication}

Several communication methods can be used in the proposed prototype, either via cables or wirelessly. Keeping in mind that the prototype should be as close as possible to a real quadcopter, the latter method was chosen.

Bluetooth, WiFi and Radio modules are some of the options considered, from which the first one was selected; more specifically, the Arduino compatible HC-05 Bluetooth Module presented in Figure 7a. A Bluetooth USB dongle, similar to the one presented in Figure 7b, was connected to the PC allowing it to communicate with the Arduino board via a serial port.

Figure 7: Bluetooth kit.

(a) HC-05 RS232 Module.

(b) Bluetooth USB dongle.
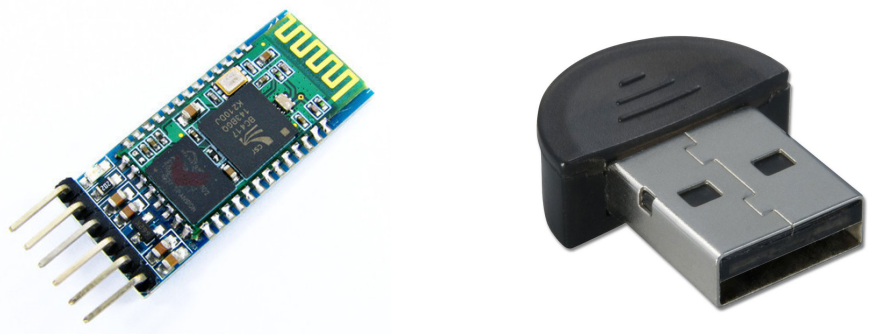

Source: (a) www.filipeflop.com, (b) www.lindy.co.uk

\subsubsection{Sensors}

The most common sensor used for an UAV is the Inertial Measurement Unit (IMU). A vast variety is available in the market, ranging from the simplest ones containing only a 
3 -axis gyroscope, to some with additional 3 -axis accelerometer, to the $10 \mathrm{DOF}$ one, which also contains a 3-axis magnetometer and a barometer.

Some years ago, most flight controllers used by hobbyists in multirotors had the task of controlling the rotation speed of the vehicle, not its angles, as it used only gyroscopes. With the inclusion of accelerometers, pitch and roll angles could be estimated and controlled, but not the yaw, because gravity has no influence in this angle. The solution was to use a magnetometer, a sensor that senses the magnetic field of the earth and provides information to calculate the angle of yaw. The barometer was included to act alongside a GPS sensor to implement the altitude hold algorithm.

Sensor fusion has to be performed in regard to estimating three angles with information from three 3-axis sensors. The goal is to use the pros of each sensor to counterbalance the cons of the others, providing reliable and robust data. Complimentarily, Kalman and Extended-Kalman filters have been proposed to this task.

The IMU chosen for this project was the GY-86 shown in Figure 8a, a 10DOF board. Accelerometers and gyroscopes are encapsulated in the MPU-6050 device (INVENSENSE INC., 2013), the magnetometer is the HMC5883L (HONEYWELL, 2013) and the barometer, MS5611 (MEASUREMENT SPECIALTIES, 2012). In short, this is a low-cost, low-power and high-performance board. Its characteristics are presented in Table 1.

Figure 8: IMU and distance sensors.

(a) GY-86 IMU.

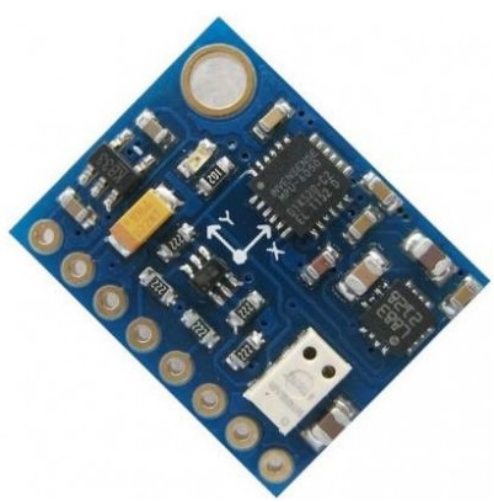

(b) GP2Y0E02A distance sensor.

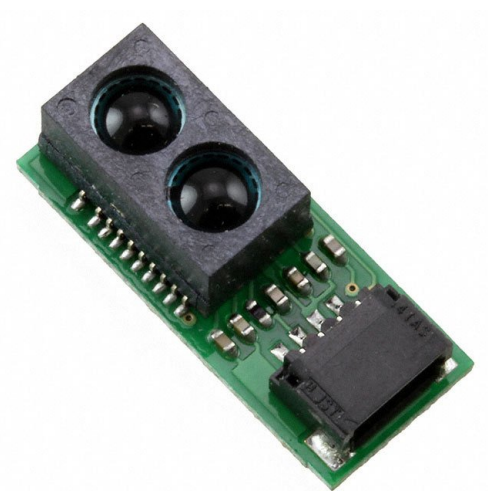

Source: (a) xcluma.com, (b) www.digikey.com

Calibration and fusion of sensors are topics of several studies throughout the world and play a major role in the efficiency of UAVs. There is an open-source project called FreeIMU (VARESANO, 2013), created by Fabio Verasano and maintained by many programmers at GitHub, aimed at creating an easy-to-use Arduino library for orientation and motion 
Table 1: IMU features

\begin{tabular}{ccc} 
Feature & Gyroscope & Accelerometer \\
\hline Full-scale range & $\pm 250, \pm 500, \pm 1000$ and $\pm 2000[\% / \mathrm{s}]$ & $\pm 2, \pm 4, \pm 8$ and $\pm 16[\mathrm{~g}]$ \\
Operating current & $3.6 \mathrm{~mA}$ & $500 \mu \mathrm{A}$ \\
ADC & $16 \mathrm{bit}$ & $16 \mathrm{bit}$ \\
\hline & & \\
Feature & Magnetometer & Barometer \\
\hline Full-scale range & $\pm 8[$ gauss] & $3 \mu \mathrm{A}$ \\
Operating current & $100 \mu \mathrm{A}$ & $24 \mathrm{bit}$ \\
ADC & $12 \mathrm{bit}$ & $\pm 1.5[\mathrm{mbar}]$ \\
Accuracy & 1 to $2\left[^{\circ}\right]$ & \\
\hline
\end{tabular}

sensing, simplifying the development of projects based upon the most powerful and new consumer grade 10DOF IMUs. FreeIMU provides a visual interface, a calibration GUI and the Arduino firmware. The first is a Processing sketch that displays a 3D cube representing the IMU and its orientation (Figure 9a); the second is a Python program that calibrates both magnetometer and accelerometer (Figure 9b); the latter is the firmware to be uploaded to the Arduino board.

Figure 9: FreeIMU GUIs.

(a) IMU and its orientation.

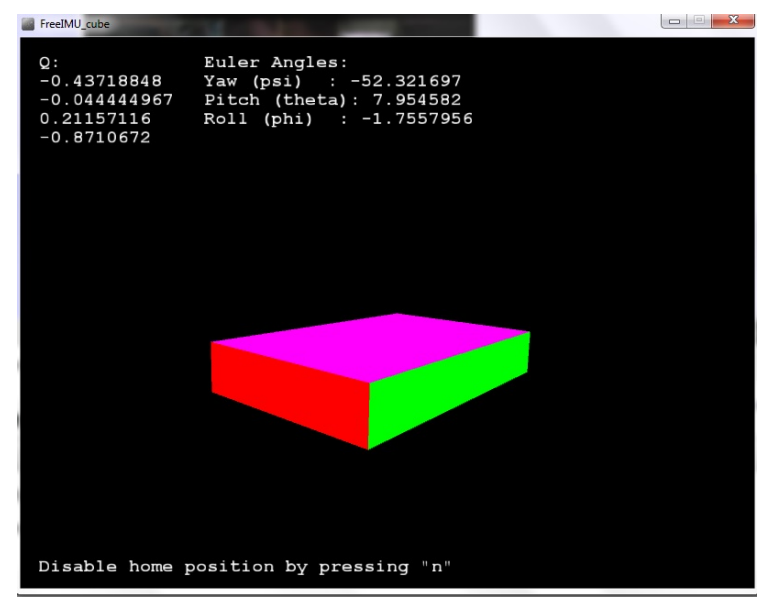

(b) Calibration GUI created with Python.

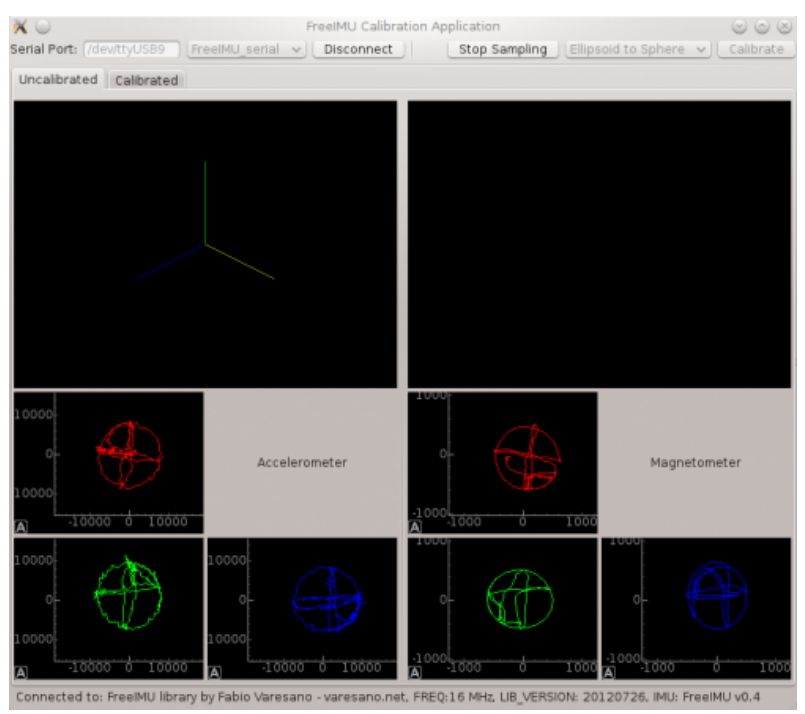

Source: www.verasano.net

One can choose among two filters to use with FreeIMU: a gradient descent algorithm (MADGWICK; HARRISON; VAIDYANATHAN, 2011) and Madgwick's implementation of Robert Mahony's DCM (direction cosine matrix) filter in quaternion form (EUSTON et al., 2008; MAHONY; HAMEL; PFLIMLIN, 2008), which was the one used in this project. 
In order to estimate the vehicle's altitude, there are several methods discussed in forums throughout the internet. The most common suggestion is to fuse barometer readings with the accelerometer z-component with a Kalman Filter and reach a $10 \mathrm{~cm}$ precision. Some more complex commercial controllers also use GPS sensors to improve precision and cancel sensor drift. However, this precision might be interesting for outdoor flights, but not for indoors, specifically not for the prototype proposed in this work.

Two possible solutions were to use ultrasonic or IR (infrared) sensors. Even though the former has a wider distance measuring, it is noisier and slower than the latter, motivating the use of the IR sensor. A GP2Y0E02A distance measuring sensor unit, presented in Figure $8 \mathrm{~b}$, was used in the project, which is able to measure distances from $4 \mathrm{~cm}$ to $50 \mathrm{~cm}$ provided by an analog output and the precision relies on the Arduino Due analog-to-digital converter.

\subsubsection{Actuators}

Brushed DC motors were used in multirotors some years ago but they have many drawbacks, such as: low power to weight ratio, high inertia, low speed and low efficiency. Bresciani (2008) had to use gearboxes to increase the speed of the propeller. Brushless DC motors are the most widely used nowadays due to its great power to weight ratio, fast response and practically constant torque; they do not have brushes to wear out and can reach impressive speeds.

Differently from the brushed motors, BLDC ones have fixed coils and rotating permanent magnets, allowing them to be built in two types: inrunners and outrunners. The former has its magnets directly attached to the shaft, while in the latter they are placed on the outer shell, as presented in Figure 10. Outrunners are capable of delivering more torque at low speeds than inrunners due to the possibility of building them with more poles (SANCA, 2013).

Choosing motor and propeller to suit an application is not an easy task. It depends on the thrust need, the power supply capacity and space available. One has to pick a combination of motor and propeller among an infinity of products for sale. For instance, operating voltages range from $8 \mathrm{~V}$ to $24 \mathrm{~V}$, motor $K v$ (constant that relates the average RPM per Volt) from 300 to 3000, and propellers from 4 to 15 inches. There are several websites and forums that give hints on how to choose wisely, while the stores start selling kits for each application. 
Figure 10: Schematics of inrunner and outrunner BLDC motors.

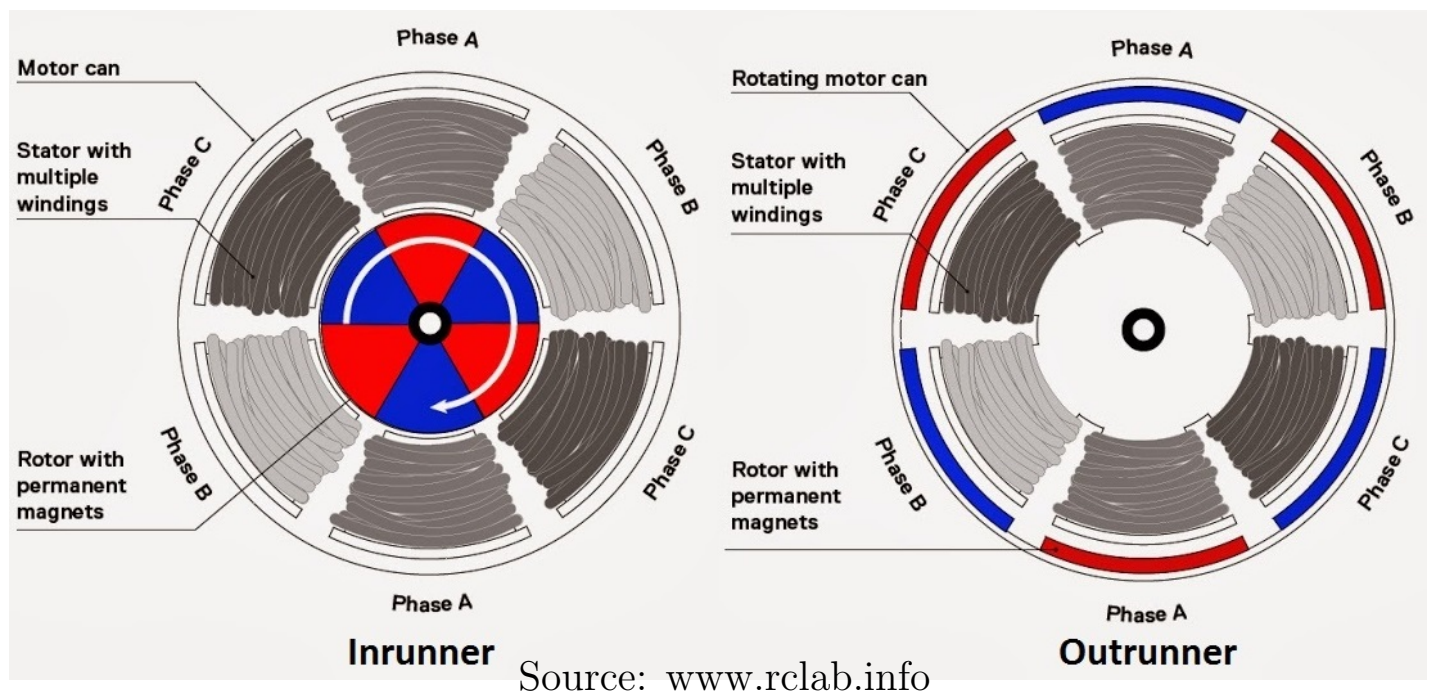

Figure 11: ESC, motor, propeller and power supply.

(a) 12A ESC and 2300Kv brushless DC motor.
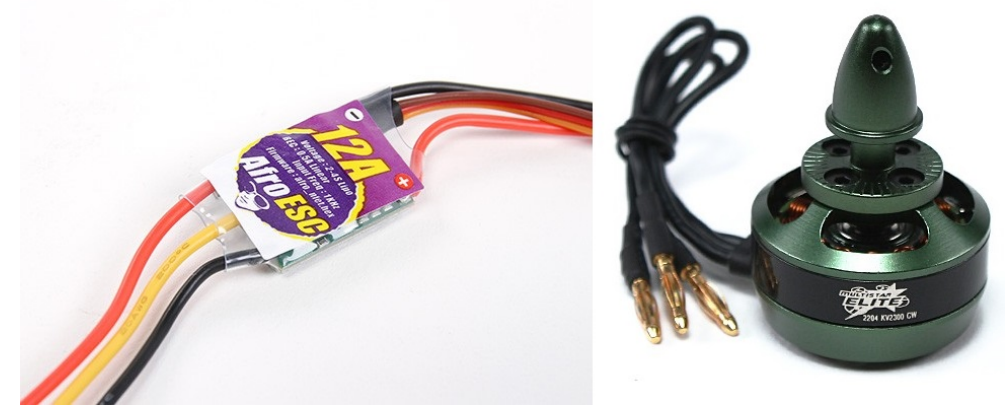

(b) 5-inch propellers and shaft adapters.

(c) $600 \mathrm{~W} 50 \mathrm{~A}$ power supply.
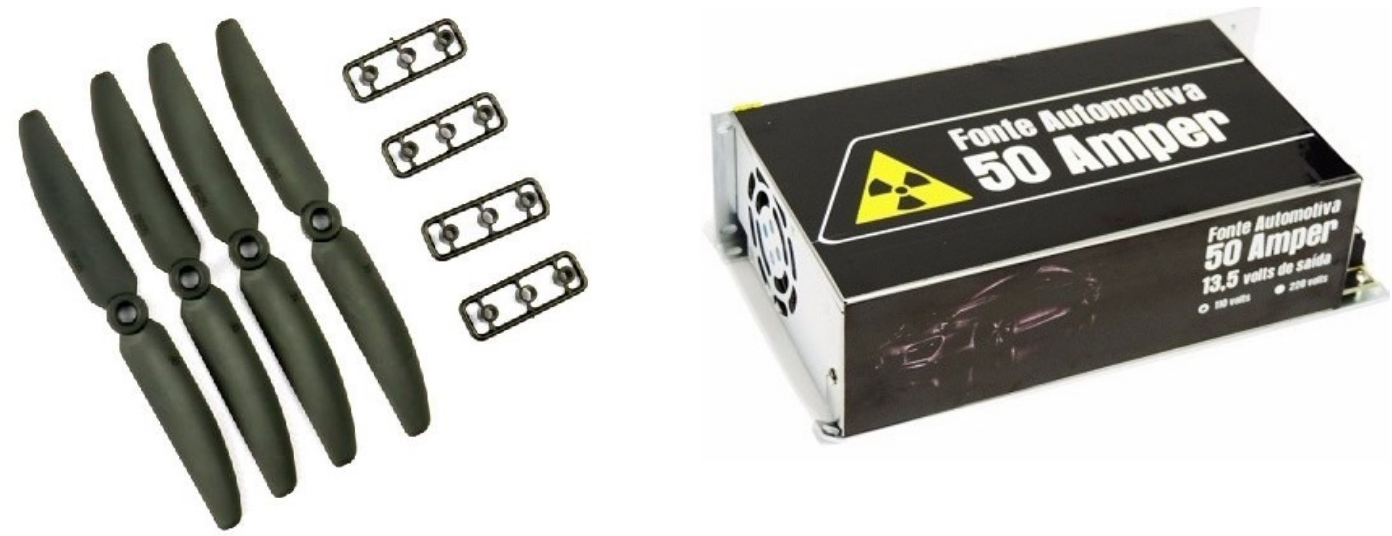

Source: (a)(b) www.hobbyking.com, (c) www.mercadolivre.com.br

The quadcopter developed here fits in the $250 \mathrm{~mm}$ FPV (first-person view) racers category, in which 5-inch propellers and over $2000 K v$ motors are used. After carefully searching for suitable motors, the MultiStar Elite $22042300 K v$, shown in Figure 11a, was chosen. It is specially developed for multirotor racers, uses 5-inch propellers and 
$12 \mathrm{~V}$ power supply. Figure 11a also shows the 12A Afro ESC, the electronic speed control needed to power the brushless motor, and Figure 11b shows an example of 5 -inch propellers with shaft adapters.

To power the system, a $12 \mathrm{~V}$ power supply was chosen rather than batteries. It must be capable of supplying at least 40A continuously, because each motor can draw up to $10 \mathrm{~A}$ continuously and $12 \mathrm{~A}$ peak. Figure $11 \mathrm{c}$ presents the power supply chosen, a $600 \mathrm{~W}$ one able to handle up to $50 \mathrm{~A}$ at $12 \mathrm{~V}$.

\subsubsection{Arduino Due}

The Arduino Due Development Board is based on a 32-bit ARM Cortex-M3 microcontroller. It has 54 digital input/output pins, 12 analog inputs, $512 \mathrm{~KB}$ of flash memory, $96 \mathrm{~KB}$ SRAM and $84 \mathrm{MHz}$ clock.

This board was chosen not only for being 32-bit, but because the most common Arduino version (Uno Rev3) does not have enough space for the FreeIMU.

\subsection{System Parameters}

The first step in this identification process was to use a digital scale to measure the weight of each component, which is presented in Table 2, and then using it to obtain the body inertias with CAD software: $I_{x}=I_{y}=2.257 \times 10^{-3} \mathrm{~kg} \cdot \mathrm{m}^{2}$ and $I_{z}=4.404 \times 10^{-3} \mathrm{~kg} \cdot \mathrm{m}^{2}$. The distance from motor to motor is $l=0.257 \mathrm{~m}$.

Table 2: Parts weight

\begin{tabular}{cccc} 
Component & Unit weight $[\mathrm{g}]$ & Quantity & Total weight $[\mathrm{g}]$ \\
\hline BLDC motor & 27.8 & 4 & 111.2 \\
ESC & 10.4 & 4 & 41.6 \\
Propeller & 2 & 4 & 8 \\
Propeller guard & 18 & 4 & 72 \\
Frame & 92.9 & 1 & 92.9 \\
Ball Head & 33 & 1 & 33 \\
Shaft & 100 & 1 & 100 \\
\hline
\end{tabular}

It is necessary to identify the propeller thrust and torque as a function of speed and the dynamics of the motor. Figure 12 presents the sketch of a test bench designed to identify these characteristics. It consists of a L-shaped structure in which the motor is attached to one end while the other end sits on a digital scale. The structure can rotate 
around its corner so that the scale reads a weight proportional to the force applied at the other end. Assuming that the distances from the center of rotation to the motor and to the scale are equal and represented as ' $D$ ', the operation is as follows:

- Thrust mode: the force applied by the propeller creates a torque in the center of rotation, which creates a downward force on the scale equal to that of the propeller.

- Torque mode: the propeller torque $(M)$ is related to the scale reading $(F)$ as $M=$ FD.

Figure 12: Torque and thrust modes of the BLDC motor test bench.

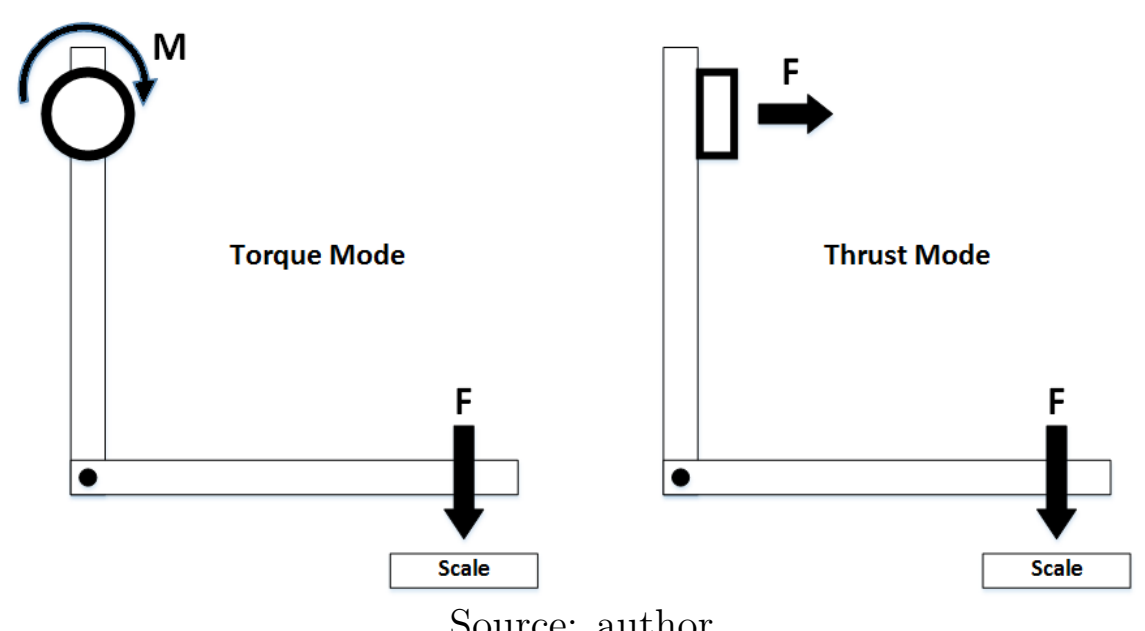

Figure 13 shows the test bench built in the laboratory. A CNY70 reflective optical sensor was used to acquire the motor rotation speed. When attached to a digital port of a microcontroller, this sensor acts as a black and white detector. Gluing a piece of white tape to the black part of the motor and counting the number of black-to-white or white-to-black changes in a time window allows calculating the average speed of rotation.

As explained in Section 2.1, the thrust and torque produced by the propellers can be approximated as a linear function to its squared speed. Figures 14 and 15 presents the experimental results performed on the test bench described above, from which $b$ and $d$ could be approximated to $6.6494 \times 10^{-7} \mathrm{~N} \cdot \mathrm{rad}^{-2} \cdot \mathrm{s}^{2}$ and $9.5206 \times 10^{-9} \mathrm{~N} \cdot \mathrm{m} \cdot \mathrm{rad}^{-2} \cdot \mathrm{s}^{2}$, respectively.

ESCs are controlled by a special type of PPM (Pulse-Position Modulation) in which the signal has to be at maximum voltage for $1 \mathrm{~ms}$ to $2 \mathrm{~ms}$, the former meaning motor stopped and the latter, top speed. In this project, this time interval is treated as a percentage of the speed, with $1 \mathrm{~ms}$ as $0 \%$ and $2 \mathrm{~ms}$ as $100 \%$. Figure 16 shows the linear 
Figure 13: Motor test bench built at LCA.

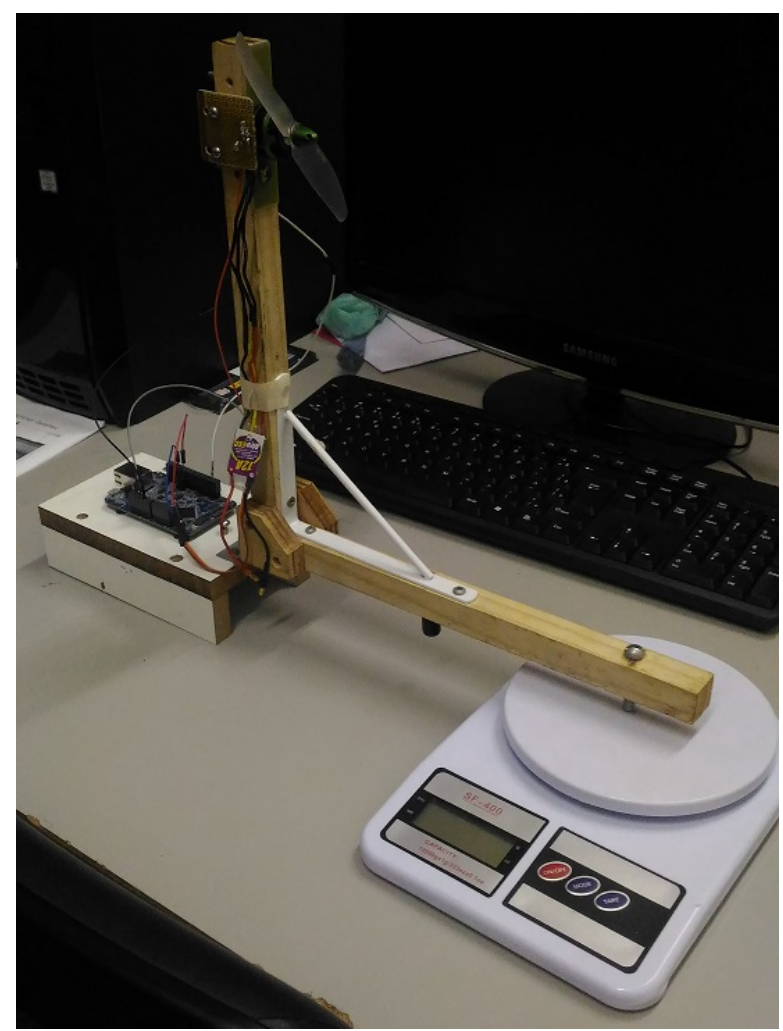

Source: author

relation between this percentage and the motor speed. In fact, the ESCs firmware were updated to a version that enables active braking and has an internal PI controller. It can be seen in Figure 17 that the motor presents fast dynamics and can be approximated by the following first-order transfer function:

$$
G(s)=\frac{29}{0.008 s+1} .
$$


Figure 14: Linear approximation to the thrust generated as a function of the squared rotational speed. The round marks are experimental results, the continuous line its approximation. The slope is $6.6494 \times 10^{-7} \mathrm{~N} \cdot \mathrm{rad}^{-2} \cdot \mathrm{s}^{2}$.

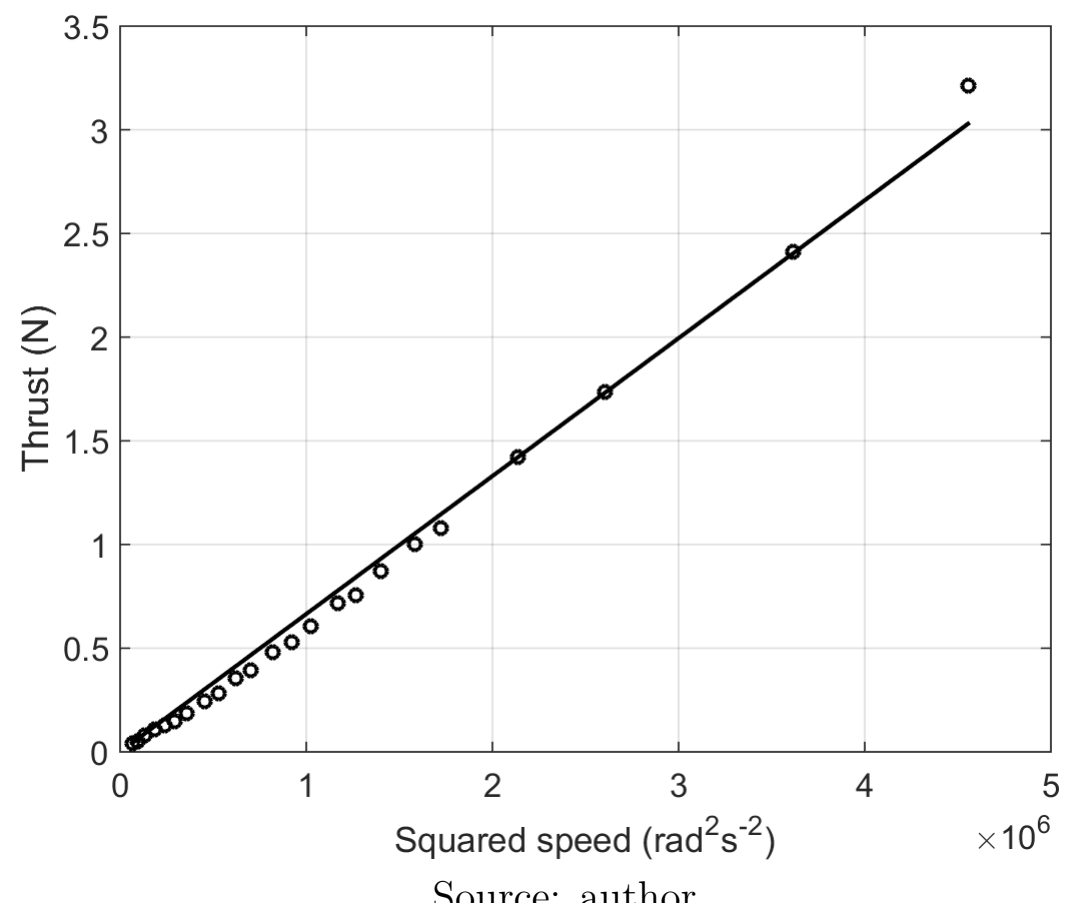

Source: author

Figure 15: Linear approximation to the torque generated as a function of the squared rotational speed. The round marks are experimental results, the continuous line its approximation. The slope is $9.5206 \times 10^{-9} \mathrm{~N} \cdot \mathrm{m} \cdot \mathrm{rad}^{-2} \cdot \mathrm{s}^{2}$.

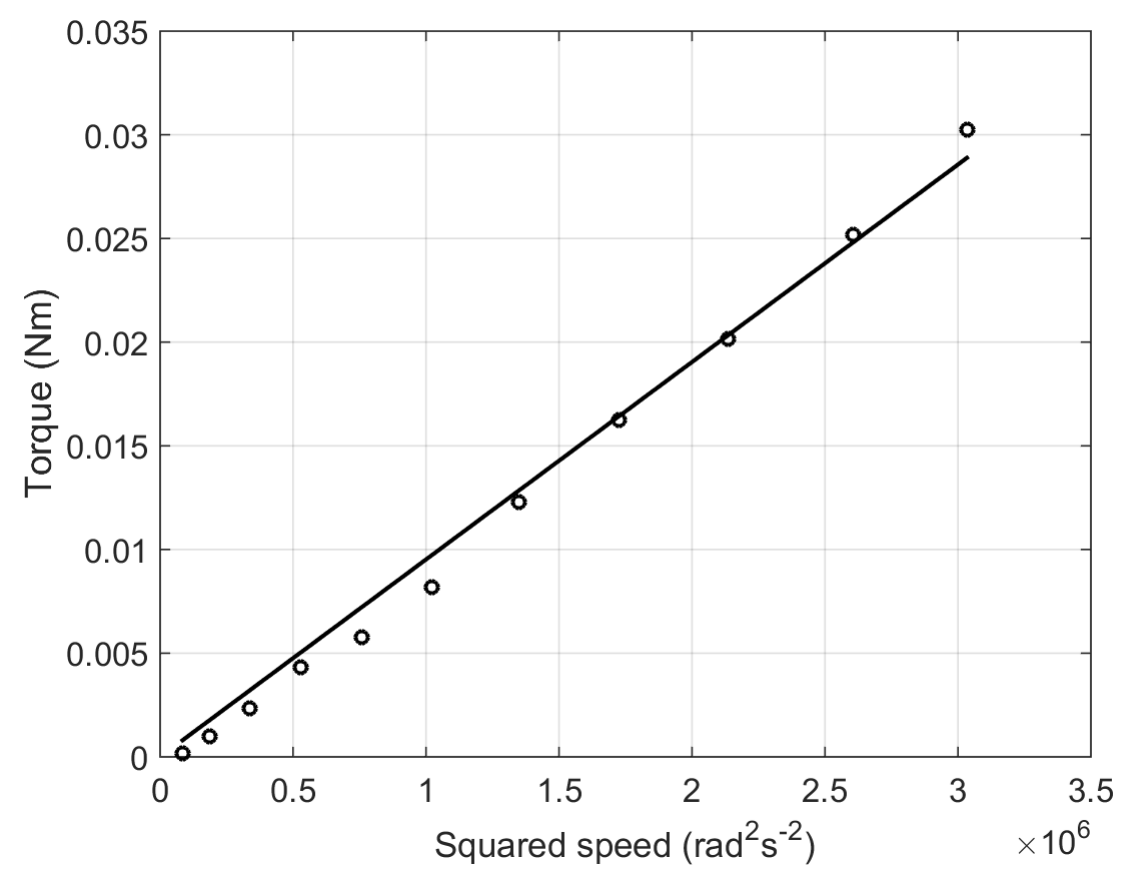

Source: author 
Figure 16: ESC signal percentage as a function of the motor speed. The round marks are experimental results, the continuous line its approximation. The slope is $3.44806 \times$ $10^{-2 \%} \cdot \mathrm{rad}^{-1} \cdot s$.

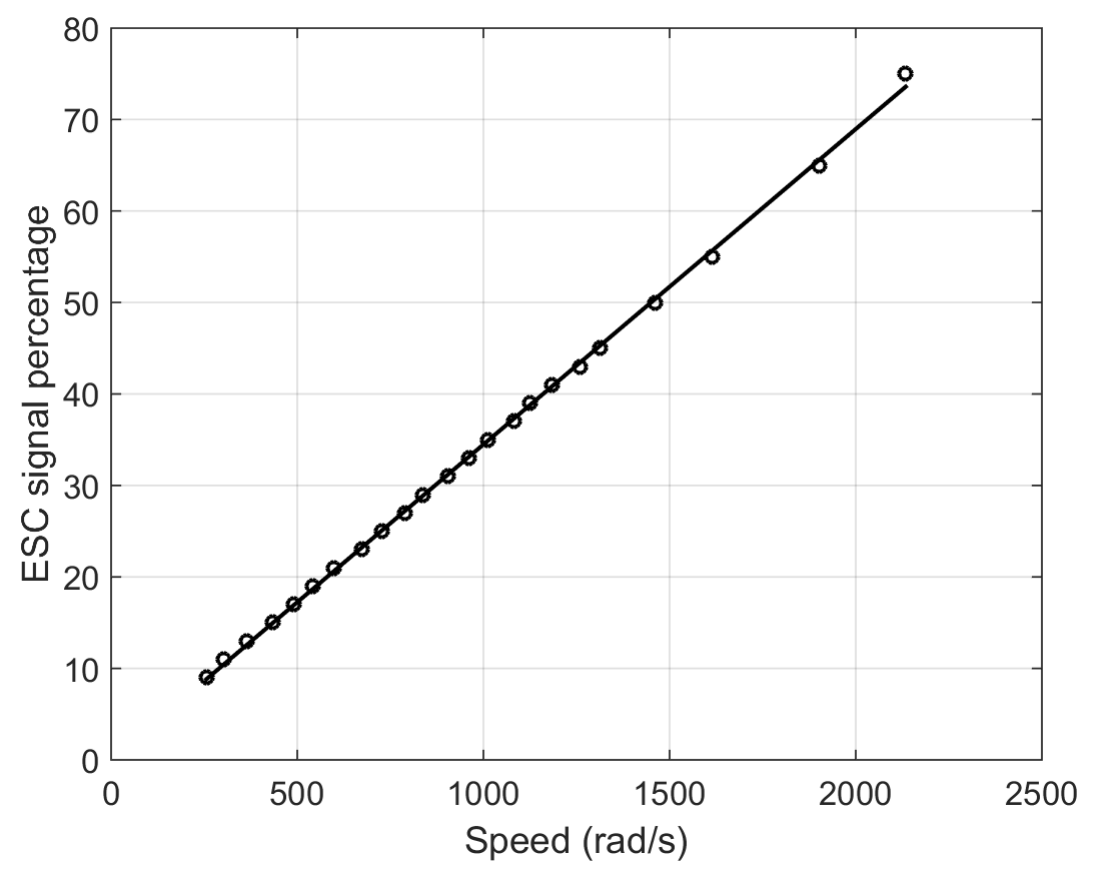

Source: author

Figure 17: Step response of the motor/propeller system. The reference starts at $40 \%$, goes to $50 \%$ and returns to $40 \%$.

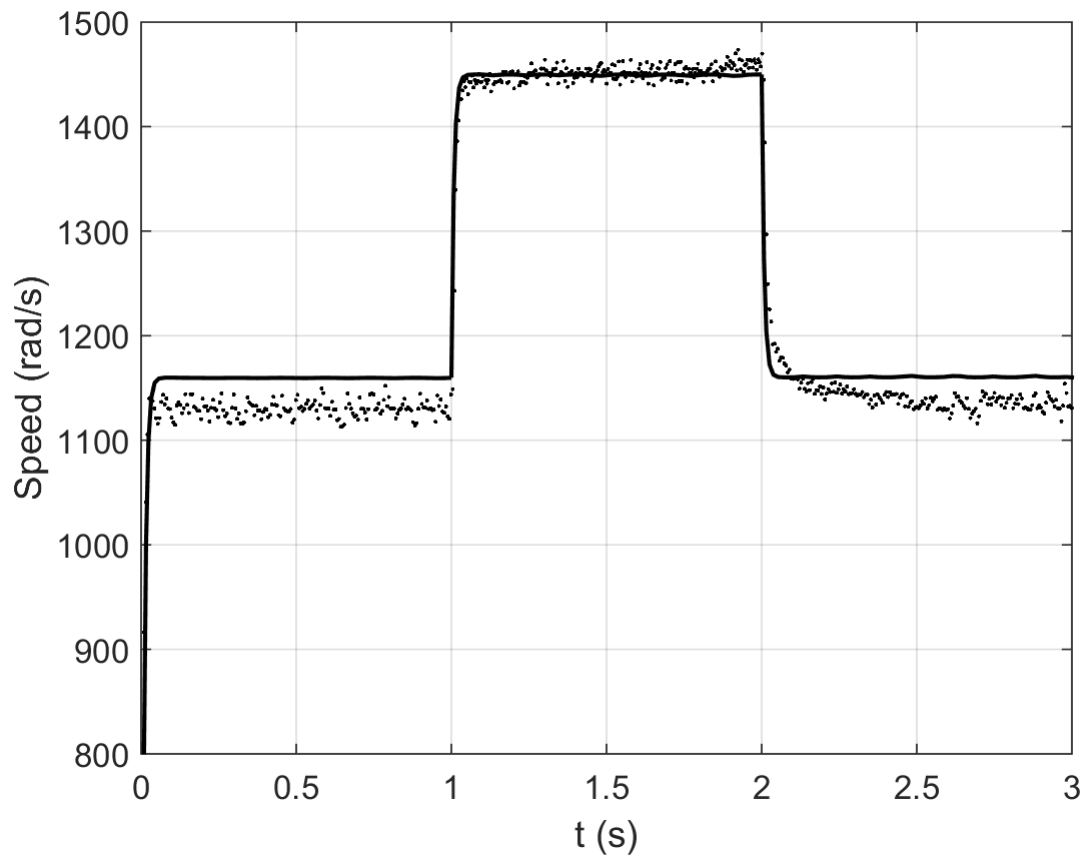

Source: author 


\section{CONTROL TECHNIQUES}

Four controllers were chosen to be studied and applied in this project: two linears (PID and LQR) and two nonlinears (Sliding Mode and Integral Sliding Mode with LQR). This chapter is intended to present the basic theory about them.

\subsection{PID controller}

The main references used in this sub-chapter are Castrucci, Bittar and Sales (2011), Ogata (2003) and Fadali and Visioli (2012).

Even tough modern controllers theory have been developed and are widely applied among academic works, the proportional-integral-derivative (PID) controller still stands as the most used for industrial applications. This is also valid for commercial multirotors and open-source flight controllers.

The ISA (International Society of Automation) equation of a PID controller is

$$
u(t)=K_{c}\left(e(t)+\frac{1}{T_{I}} \int_{0}^{t} e(\tau) d \tau+T_{D} \frac{d e(t)}{d t}\right)
$$

in which $u(t)$ is the controller output, $e(t)$ is the controller input given by $e(t)=r(t)-y(t)$, $r(t)$ is the reference signal, $y(t)$ is the system output and $K_{c}, K_{c} / T_{I}$ and $K_{c} T_{D}$ are the proportional, integral and derivative gains, respectively, with $T_{I}$ being the reset time and $T_{D}$ the derivative time. Figure 18 shows the block diagram corresponding to the PID transfer function

$$
P I D(s)=\frac{U(s)}{E(s)}=K_{c}\left(1+\frac{1}{T_{I} s}+T_{D} s\right)
$$

The derivative term in this equation has two undesirable characteristics. The first is on deriving the error: when applied to systems with varying step references, this derivative term may cause peaks in the controller output. The solution is deriving only the plant output and not subtracting it from the reference, as presented in Figure 19 and in the 
Figure 18: ISA form PID block diagram.

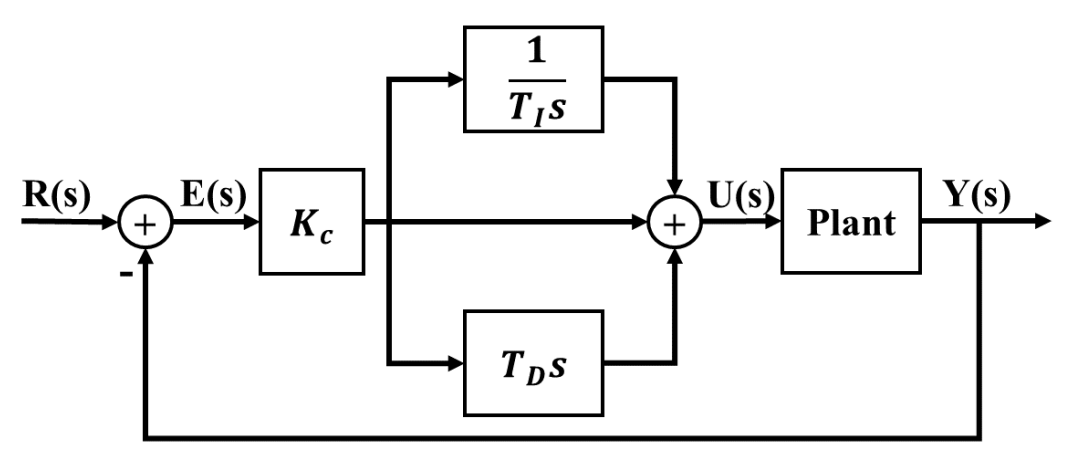

Source: author

Figure 19: PID block diagram with output derivative.

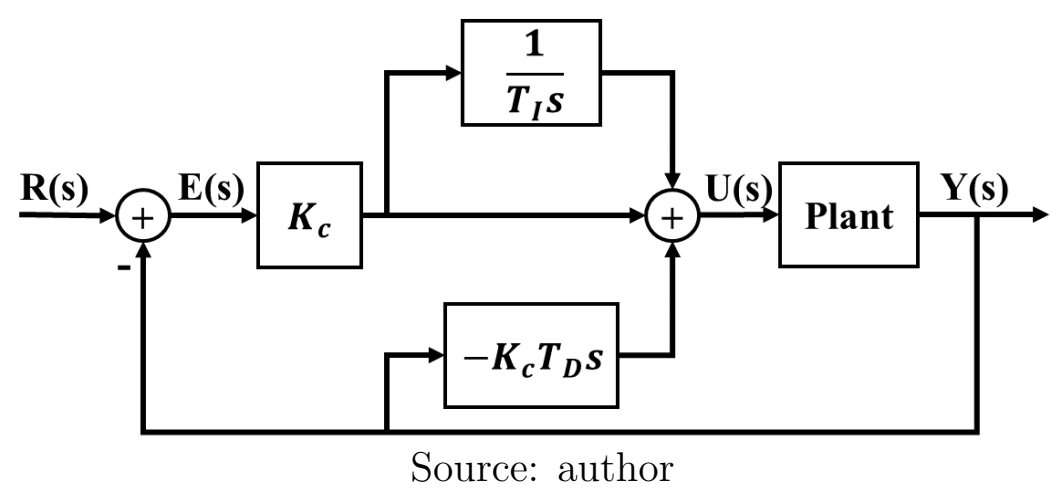

following equation. This is also known as PI+D.

$$
U(s)=K_{c}\left(1+\frac{1}{T_{I} s}\right) E(s)-K_{c} T_{D} s Y(s)
$$

The second problem is that this derivative gain tends to infinity as frequency raises, i.e., it amplifies high frequency noise. The solution is to add a low-pass filter to it, as shown in the next equation, resulting in a realistic PID form.

$$
U(s)=K_{c}\left(1+\frac{1}{T_{I} s}\right) E(s)-\frac{K_{c} T_{D} s}{\left(T_{D} / N\right) s+1} Y(s)
$$

If it is possible to have sensors measuring the derivative of the plant output, such as gyroscopes for angular velocity, a different PID controller can be implemented, which we may call "derivative-free". The idea is to avoid deriving a noise signal by feedbacking the output derivative, as shown next

$$
U(s)=K_{c}\left(1+\frac{1}{T_{I} s}\right) E(s)-\frac{K_{c} T_{D}}{\left(T_{D} / N\right) s+1} \dot{Y}(s) .
$$




\subsubsection{Discretization}

Since the controller will be implemented in a microcontroller, it is necessary to write it as difference equations by discretizing it. The PID equation can be divided into the following three equations:

$$
\begin{aligned}
U_{P}(s) & =K_{c} E(s), \\
U_{D}(s) & =-\frac{K_{c} T_{D} s}{\left(T_{D} / N\right) s+1} Y(s), \\
U_{I}(s) & =\frac{K_{c}}{T_{I} s} E(s) .
\end{aligned}
$$

Discretizing the proportional part simply results in $u_{P}[n]=K_{c} e[n]$. Regarding the derivative part, Backward Euler method can be used, such that

$$
s=\frac{z-1}{T_{s} z}
$$

in which $z$ is the $z$-transform and $T_{s}$ the sampling period. By doing so,

$$
U_{D}(z)=-\frac{K_{c} N T_{D}(z-1)}{\left(T_{D}+N T_{s}\right) z-T_{D}} Y(z)=-\frac{K_{c} N T_{D}\left(1-z^{-1}\right)}{\left(T_{D}+N T_{s}\right)-T_{D} z^{-1}} Y(z) .
$$

Anti-transforming it results in

$$
u_{D}[n]=\frac{T_{D}}{T_{D}+N T_{s}} u_{D}[n-1]-\frac{K_{c} N T_{D}}{T_{D}+N T_{s}}(y[n]-y[n-1]) .
$$

Using Backward Euler method to also discretize the integrative part results in

$$
U_{I}(z)=\frac{K_{c} T_{s} z}{T_{I}(z-1)} E(z)=\frac{K_{c} T_{s}}{T_{I}\left(1-z^{-1}\right)} E(z),
$$

which difference equation is written as

$$
u_{I}[n]=u_{I}[n-1]+\frac{K_{c} T_{s}}{T_{I}} e[n] .
$$

At last, the "derivative-free" PID is discretized as

$$
U_{D}(z)=-\frac{K_{c} N T_{D} T_{s} z}{\left(T_{D}+N T_{s}\right) z-T_{D}} \dot{Y}(z)=-\frac{K_{c} N T_{D} T_{s}}{\left(T_{D}+N T_{s}\right)-T_{D} z^{-1}} \dot{Y}(z),
$$

and

$$
u_{D}[n]=\frac{T_{D}}{T_{D}+N T_{s}} u_{D}[n-1]-\frac{K_{c} N T_{D} T_{s}}{T_{D}+N T_{s}} \dot{y}[n]
$$




\subsection{LQR Controller}

The Linear Quadratic Regulator is an optimal controller designed in state-space such that the system to be regulated can be written as

$$
\begin{aligned}
\dot{\mathbf{x}}(t) & =\mathbf{A} \mathbf{x}(t)+\mathbf{B u}(t) \\
\mathbf{y}(t) & =\mathbf{C} \mathbf{x}(t),
\end{aligned}
$$

with $\mathbf{x}(t)$ being the state vector, $\mathbf{y}(t)$ the output vector, $\mathbf{u}(t)$ the control signal and A, $\mathbf{B}$ and $\mathbf{C}$ the state, input and output matrices, respectively. The control signal is $\mathbf{u}(t)=-\mathbf{K} \mathbf{x}(t)$ with $\mathbf{K}$ such that the following cost function is minimized

$$
J=\int_{0}^{\infty}\left(\mathbf{x}^{T}(t) \mathbf{Q} \mathbf{x}(t)+\mathbf{u}^{T}(t) \mathbf{R u}(t)\right) d t
$$

in which $\mathbf{Q}$ is a real symmetric positive semi-definite matrix and $\mathbf{R}$ is a real symmetric positive definite matrix, and they define the relative importance of the states error and the energy consumption by the controller. This is known as Regulator Form, as presented in Figure 20, since the objective is stabilizing the states at the linearization point.

Figure 20: Block diagram of the LQR in regulation form.

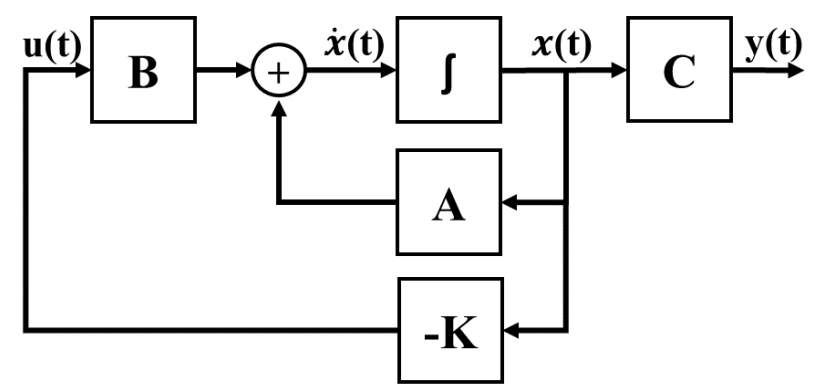

Source: author

As a matter of fact, it is possible to prove that the optimal control law that satisfies the quadratic performance index is given by (OGATA, 2003; GEROMEL; KOROGUI, 2011)

$$
\mathbf{u}(t)=-\mathbf{K} \mathbf{x}(t)=-\mathbf{R}^{-1} \mathbf{B}^{T} \mathbf{P} \mathbf{x}(t),
$$

with $\mathbf{P}$ being the solution of the following Riccati equation

$$
\mathbf{A}^{T} \mathbf{P}+\mathbf{P A}-\mathbf{P B R}^{-1} \mathbf{B}^{T} \mathbf{P}+\mathbf{Q}=\mathbf{0} .
$$

Geromel and Korogui (2011) also show that, for single-input systems with $\mathbf{Q}=\mathbf{I}$ and 
$\mathbf{R}=\rho$, the left-plane eigenvalues (negative real part) of the matrix $\mathbf{Z}$ written as

$$
\mathbf{Z}=\left[\begin{array}{cc}
\mathbf{A} & -\rho^{-1} \mathbf{B B}^{T} \\
-\mathbf{Q} & -\mathbf{A}^{T}
\end{array}\right]
$$

are the eigenvalues of the closed-loop matrix $\left(\mathbf{A}-\rho^{-1} \mathbf{B B}^{T} \mathbf{P}\right)$, therefore for the closedloop system. One can use this to design a controller that satisfies some specifications.

Integrators can be inserted to allow the system to track a reference, as presented in Figure 21. The control signal is now defined as

$$
\mathbf{u}(t)=-\mathbf{K} \mathbf{x}(t)+\mathbf{K}_{\mathbf{I}} \mathbf{v}(t)
$$

and the integrator equation is

$$
\dot{\mathbf{v}}(t)=\mathbf{r}(t)-\mathbf{y}(t)=\mathbf{r}(t)-\mathbf{C x}(t)
$$

Figure 21: Block diagram of the LQR with integrator insertion.

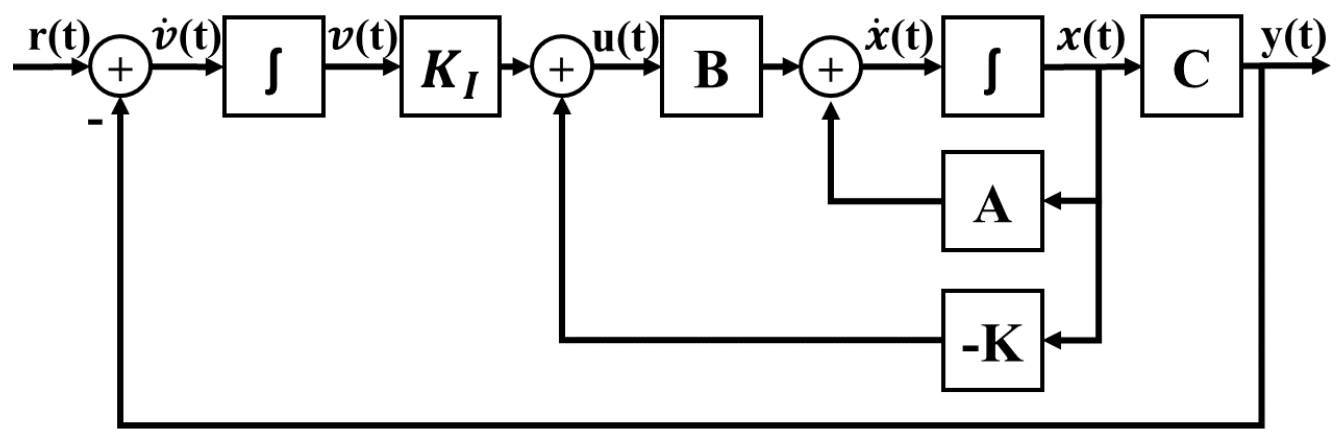

Source: author

The closed-loop equation can be written as

$$
\left[\begin{array}{c}
\dot{\mathbf{x}}(t) \\
\dot{\mathbf{v}}(t)
\end{array}\right]=\left[\begin{array}{cc}
\mathbf{A}-\mathbf{B K} & \mathbf{B K} \mathbf{I} \\
-\mathbf{C} & \mathbf{0}
\end{array}\right]\left[\begin{array}{l}
\mathbf{x}(t) \\
\mathbf{v}(t)
\end{array}\right]+\left[\begin{array}{l}
\mathbf{0} \\
\mathbf{I}
\end{array}\right] \mathbf{r}(t),
$$

from which it is possible to show that

$$
\left[\begin{array}{cc}
\mathbf{A}-\mathbf{B K} & \mathbf{B K}_{\mathbf{I}} \\
-\mathbf{C} & \mathbf{0}
\end{array}\right]=\underbrace{\left[\begin{array}{cc}
\mathbf{A} & \mathbf{0} \\
-\mathbf{C} & \mathbf{0}
\end{array}\right]}_{\mathbf{A}_{a u g}}-\underbrace{\left[\begin{array}{c}
\mathbf{B} \\
\mathbf{0}
\end{array}\right]}_{\mathbf{B}_{a u g}} \underbrace{\left[\begin{array}{rr}
\mathbf{K} & -\mathbf{K}_{\mathbf{I}}
\end{array}\right]}_{\mathbf{K}_{a u g}} .
$$

Thus, an LQR controller can be designed for the augmented state-space system defined 
by matrices $\mathbf{A}_{a u g}, \mathbf{B}_{a u g}$ and $\mathbf{C}_{a u g}=\left[\begin{array}{ll}\mathbf{C} & \mathbf{0}\end{array}\right]$. The control signal is then defined as

$$
\mathbf{u}(t)=-\mathbf{K}_{a u g} \mathbf{x}_{a u g}(t)
$$

\subsubsection{Discretization}

As the LQR presented is a fixed-gain feedback controller, it has no changes when discretized. Then,

$$
\mathbf{u}[n]=-\mathbf{K}_{a u g} \mathbf{x}_{a u g}[n]
$$

However, integrators have been inserted on this augmented system, and they have to be discretized. Using the Backward Euler method, one can write

$$
\mathbf{v}[n]=\mathbf{v}[n-1]+T_{s}(\mathbf{r}[n]-\mathbf{y}[n])
$$

If using the Forward Euler method, the equation becomes

$$
\mathbf{v}[n+1]=\mathbf{v}[n]+T_{s}(\mathbf{r}[n]-\mathbf{y}[n])
$$

\subsection{Sliding Mode Controller}

The Sliding Mode Controller is a nonlinear controller that presents good robustness characteristics, mainly on modeling inaccuracies on the terms of the equation (parametric) or admitted for simplicity (unmodeled). The main reference used to developed this text is Slotine and $\mathrm{Li}$ (1991). Let a single-input system be written as

$$
x^{(n)}=f(\boldsymbol{x})+b(\boldsymbol{x}) u
$$

where $x$ is the output of interest, $n$ is its $n$-th derivative, $u$ is the control input and $\boldsymbol{x}=\left[\begin{array}{llll}x & \dot{x} & \ldots & x^{(n-1)}\end{array}\right]^{T}$ is the state vector. Still in this equation, $f(\boldsymbol{x})$ is a nonlinear function not precisely known and $b(\boldsymbol{x})$ has its sign known, but also presents imprecision.

The main objective is to make the state vector $\boldsymbol{x}$ track a desired varying state $\boldsymbol{x}_{d}=\left[\begin{array}{llll}x_{d} & \dot{x}_{d} & \ldots & x_{d}^{(n-1)}\end{array}\right]^{T}$. Let us start by creating a tracking error vector $\tilde{\boldsymbol{x}}$ such that

$$
\tilde{\boldsymbol{x}}=\boldsymbol{x}-\boldsymbol{x}_{d}=\left[\begin{array}{llll}
\tilde{x} & \dot{\tilde{x}} & \ldots & \tilde{x}^{(n-1)}
\end{array}\right]^{T}
$$


and then a surface in $\boldsymbol{R}^{n}$ can be defined as

$$
s(\boldsymbol{x} ; t)=\left(\frac{d}{d t}+\lambda\right)^{n-1} \tilde{x} .
$$

In fact, given the initial condition $\boldsymbol{x}_{d}(0)=\boldsymbol{x}(0)$, the problem of making $\tilde{\boldsymbol{x}}=0$ is equivalent to that of staying on this surface. Thus, the $n$-dimensional tracking problem was reduced to a first order in $s$, and differentiating it once already makes the control input appear. The following equation shows that bonds on $s$ can be directly translated into bonds on the tracking vector:

$$
|s(t)| \leq \Phi \rightarrow\left|\tilde{x}^{(i)}(t)\right| \leq(2 \lambda)^{i} \frac{\Phi}{\lambda^{n-1}}, i=0, \ldots, n-1 .
$$

In case of initial tracking error different than zero, i.e., $\tilde{\boldsymbol{x}}(0) \neq \mathbf{0}$, it is possible to show that this bound is obtained asymptotically with time constant $(n-1) / \lambda$. The sliding condition, given by

$$
\frac{1}{2} \frac{d}{d t} s^{2} \leq-\eta|s|
$$

with $\eta$ a strictly positive constant, makes the surface an invariant set and once on it, the trajectories remain on it. At last, it can be proved that if outside the surface, it will be reached with finite time smaller than $|s(0)| / \eta$ and, once on the surface, the tracking error will exponentially tend to zero with time constant $(n-1) / \lambda$.

By assuming that $f(\boldsymbol{x})$ is not precisely known but estimated by $\hat{f}(\boldsymbol{x})$ such that the estimation error is

$$
|\hat{f}(\boldsymbol{x})-f(\boldsymbol{x})| \leq F
$$

and that $b(\boldsymbol{x})$ is bounded by $0<b_{\min } \leq b(\boldsymbol{x}) \leq b_{\max }$ and estimated as

$$
\hat{b}(\boldsymbol{x})=\sqrt{b_{\min } b_{\max }}
$$

one can write the following control law

$$
u=\hat{b}^{-1}(\boldsymbol{x})[\hat{u}-k \operatorname{sgn}(s)]
$$

in which a second-order $(n=2)$ sliding surface is defined as

$$
s=\left(\frac{d}{d t}+\lambda\right) \tilde{x}=\dot{\tilde{x}}+\lambda \tilde{x}
$$


and its derivative is

$$
\begin{aligned}
\dot{s} & =\ddot{\tilde{x}}+\lambda \dot{\tilde{x}} \\
& =\ddot{x}-\ddot{x}_{d}+\lambda \dot{\tilde{x}} \\
& =f(\boldsymbol{x})+b(\boldsymbol{x}) u-\ddot{x}_{d}+\lambda \dot{\tilde{x}}
\end{aligned}
$$

Since uncertainties are being considered, the best approximation for the control input is

$$
\hat{u}=-\hat{f}(\boldsymbol{x})+\ddot{x}_{d}-\lambda \dot{\tilde{x}}
$$

At last, $k$ is chosen such that the sliding condition is satisfied, i.e.,

$$
k \geq \beta(F+\eta)+(\beta-1)|\hat{u}|
$$

with $\beta=\sqrt{b_{\max } / b_{\min }}$. Figure 22 presents the block diagram for this controller.

Figure 22: Block diagram of the Sliding Mode controller.

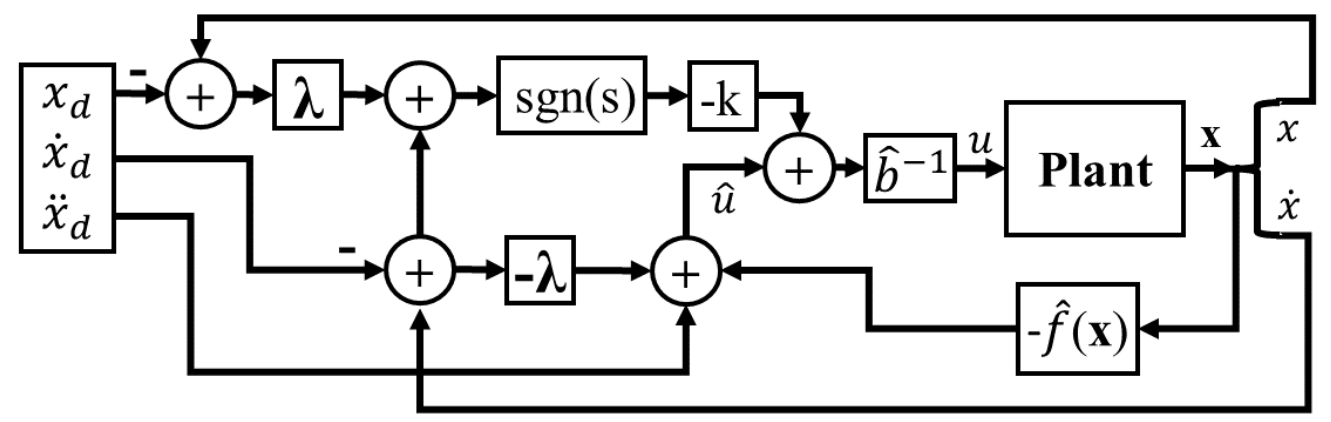

Source: author

Even though implementing the controller with the sign function provides the system perfect tracking, it causes chattering, which is not usually desirable. The solution is to use continuous approximations of switching control laws, such as the saturation function for a boundary layer $\Phi$. Thus,

$$
u=\hat{b}^{-1}(\boldsymbol{x})[\hat{u}-k \operatorname{sat}(s / \Phi)]
$$

which guarantees a tracking precision of $\Phi / \lambda^{n-1}$. 


\subsection{Integrative Sliding Mode Controller with LQR}

Slotine and Li (1991) also propose an Integral Sliding Mode controller, with surface given in the following form:

$$
\sigma(t)=\dot{\tilde{x}}(t)+2 \lambda \tilde{x}(t)+\lambda^{2} \int_{0}^{t} \tilde{x}(\tau) d \tau,
$$

with $\lambda$ a design positive constant that drives the surface to zero and $\tilde{x}(t)=x(t)-x^{d}(t)$, i.e., the difference between the actual state and the desired one. This equation can be rearranged to

$$
\sigma(t)=\left[\begin{array}{lll}
2 \lambda & 1 & \lambda^{2}
\end{array}\right]\left[\begin{array}{c}
\tilde{x}(t) \\
\dot{\tilde{x}}(t) \\
\int_{0}^{t} \tilde{x}(\tau) d \tau
\end{array}\right]
$$

which has two interesting characteristics:

- it is in the form $\sigma(t)=\mathbf{G x}(t)$, another SMC design methodology based on the linear states of the system (HAMAYUN; EDWARDS; ALWI, 2016);

- the state vector is similar to the one used on Linear Quadratic Regulators (LQR) with integrators inserted at the input.

As a result of our research, this subsection proposes a controller setup based on Integral Sliding Modes and LQR that guarantees null tracking error for step inputs without the use of the second derivative of the reference.

Let a Linear Time-Invariant (LTI) system, already augmented with integrators as presented in Section 3.2, be written as

$$
\dot{\mathbf{x}}_{a u g}(t)=\mathbf{A}_{a u g} \mathbf{x}_{a u g}(t)+\mathbf{B}_{a u g} \mathbf{u}(t)+\mathbf{B}_{a u g} \mathbf{D} \xi(t, x)
$$

with $\mathbf{x}_{\text {aug }}(t)$ being the state vector, $\mathbf{u}(t)$ the control signal, $\mathbf{A}_{\text {aug }}$ and $\mathbf{B}_{\text {aug }}$ the state and input matrices, respectively, and $\xi(t, x)$ represents the model uncertainty or external disturbance. A typical sliding mode control law is

$$
\mathbf{u}(t)=\mathbf{u}_{l}(t)+\mathbf{u}_{n}(t)
$$

in which $\mathbf{u}_{l}(t)$ comes from the LQR with integrator on the input and $\mathbf{u}_{n}(t)$ from a nonlinear controller; the former is chosen to be an LQR feedback gain, while the latter corresponds 
to a switching function. Then,

$$
\mathbf{u}(t)=-\mathbf{K}_{a u g} \mathbf{x}_{a u g}(t)-\rho(t, x)\left(\mathbf{G B}_{a u g}\right)^{-1} \frac{\sigma(t)}{\|\sigma(t)\|},
$$

and $\sigma(t)$ is the sliding surface, $\mathbf{G}$ is a matrix to be designed such that $\left(\mathbf{G B}_{a u g}\right)^{-1}$ is invertible and $\rho(t, x)$ such that guarantees the sliding condition.

Let us propose a surface $\sigma(t)$ dependent on the tracking error, i.e., on the difference between the actual states $\mathbf{x}_{\text {aug }}(t)$ and the desired ones, $\mathbf{x}_{\text {aug }}^{d}(t)$ :

$$
\sigma(t)=\mathbf{G}\left(\mathbf{x}_{\text {aug }}(t)-\mathbf{x}_{\text {aug }}^{d}(t)\right)
$$

Deriving this equation yields

$$
\begin{aligned}
\dot{\sigma}(t) & =\mathbf{G}\left[\dot{\mathbf{x}}_{a u g}(t)-\dot{\mathbf{x}}_{\text {aug }}^{d}(t)\right] \\
& =\mathbf{G}\left[\mathbf{A}_{\text {aug }} \mathbf{x}_{a u g}(t)+\mathbf{B}_{a u g} \mathbf{u}_{l}(t)+\mathbf{B}_{a u g} \mathbf{u}_{n}(t)+\mathbf{B}_{a u g} \mathbf{D} \xi(t, x)-\dot{\mathbf{x}}_{a u g}^{d}(t)\right],
\end{aligned}
$$

and replacing (3.49) on it results, after some mathematical simplification, in

$$
\dot{\sigma}(t)=\mathbf{G}\left[\left(\mathbf{A}_{a u g}-\mathbf{B}_{a u g} \mathbf{K}_{a u g}\right) \mathbf{x}_{a u g}(t)-\dot{\mathbf{x}}_{a u g}^{d}(t)\right]-\rho(t, x) \frac{\sigma(t)}{\|\sigma(t)\|}+\mathbf{G B}_{a u g} \mathbf{D} \xi(t, x) .
$$

The linear controller should guarantee that the first part of this equation tends to zero, allowing us to simplify it to

$$
\dot{\sigma}(t)=-\rho(t, x) \frac{\sigma(t)}{\|\sigma(t)\|}+\mathbf{G B}_{a u g} \mathbf{D} \xi(t, x) .
$$

Multiplying (3.54) on the left by $\sigma^{T}(t)$ and using the fact that $\sigma^{T}(t) \sigma(t)=\|\sigma(t)\|^{2}$ gives us

$$
\begin{aligned}
\sigma^{T}(t) \dot{\sigma}(t) & =-\rho(t, x)\|\sigma(t)\|+\sigma^{T}(t) \mathbf{G B}_{a u g} \mathbf{D} \xi(t, x) \\
& \leq\|\sigma(t)\|\left(-\rho(t, x)+\left\|\mathbf{G B}_{a u g} \mathbf{D} \xi(t, x)\right\|\right)
\end{aligned}
$$

Given the multivariable version of the Sliding Condition ( $\eta$-reachability) (HAMAYUN; EDWARDS; ALWI, 2016)

$$
\sigma^{T}(t) \dot{\sigma}(t) \leq-\eta\|\sigma(t)\|
$$

with $\eta$ a positive constant, this condition is guaranteed by choosing $\rho(t, x)$ as

$$
\rho(t, x) \geq\left\|\mathbf{G B}_{a u g} \mathbf{D} \xi(t, x)\right\|+\eta
$$




\subsubsection{Defining Matrix G}

For a single-input single-output (SISO) system, (3.50) can be written as

$$
\sigma(t)=\left[\begin{array}{lll}
G_{1} & G_{2} & G_{3}
\end{array}\right]\left[\begin{array}{c}
\tilde{x}(t) \\
\dot{\tilde{x}}(t) \\
\int_{0}^{t} \tilde{x}(\tau) d \tau
\end{array}\right]
$$

which, when changing to state variables, becomes

$$
\sigma(t)=G_{1} x_{1}(t)+G_{2} x_{2}(t)+G_{3} x_{3}(t)
$$

and knowing that $\dot{x}_{3}(t)=x_{1}(t)$ and $\ddot{x}_{3}(t)=\dot{x}_{1}(t)=x_{2}(t)$ gives us the following

$$
\sigma(t)=G_{2} \ddot{x}_{3}(t)+G_{1} \dot{x}_{3}(t)+G_{3} x_{3}(t)
$$

from which is possible to state that the sliding manifold responds as a second order system. Thus, matrix $\mathbf{G}$ defines the manifold dynamics.

For multivariable systems, one way of choosing $\mathbf{G}$ would be such that it generates uncoupled manifolds, even for coupled systems. As an example, let us define a system with two outputs, $a(t)$ and $b(t)$. Their sliding manifolds can be written as

$$
\sigma(t)=\left[\begin{array}{cccccc}
G_{1}^{a} & 0 & G_{2}^{a} & 0 & G_{3}^{a} & 0 \\
0 & G_{1}^{b} & 0 & G_{2}^{b} & 0 & G_{3}^{b}
\end{array}\right]\left[\begin{array}{c}
\tilde{a}(t) \\
\tilde{b}(t) \\
\dot{\tilde{a}}(t) \\
\dot{\tilde{b}}(t) \\
\int_{0}^{t} \tilde{a}(\tau) d \tau \\
\int_{0}^{t} \tilde{b}(\tau) d \tau
\end{array}\right] .
$$

Another method is presented in (CASTANOS; FRIDMAN, 2006), which proposes choosing $\mathbf{G}$ in order to avoid amplifying unmatched disturbances and is given by

$$
\mathbf{G}=\left(B_{\text {aug }}^{T} B_{\text {aug }}\right)^{-1} B_{\text {aug }}^{T}
$$




\subsection{Performance Indices}

In order to be able to quantitatively compare the results of the practical tests of the controllers, it is necessary to have tools to measure their performances. The error $(e)$, i.e. the difference between reference and actual state are used in three different indices:

- Integral Absolute Error (IAE): it integrates the absolute error over time.

$$
\mathrm{IAE}=\int|e| d t
$$

- Integral Time-weighted Absolute Error (ITAE): it integrates the absolute error multiplied by the time over time.

$$
\mathrm{ITAE}=\int t|e| d t
$$

- Variance: given the error vector $A$ made of $N$ sampled data and mean $\mu$, its variance is given by

$$
V=\frac{1}{N-1} \sum_{i=1}^{N}\left|A_{i}-\mu\right|^{2}
$$




\section{CONTROLLER DESIGN, SIMULATION AND RESULTS}

This chapter initially describes and analyzes the 4DOF quadcopter simplified model with which the controllers are designed. Then, the controllability and observability of the system are studied, simulation procedure and results are described and finally discussed.

\subsection{Simplified Model}

While on its operating point, i.e., hovering, the quadcopter will present pitch and roll close to zero. Thus, the following approximations are valid for a generic $\alpha$ angle:

- $\lim _{\alpha \rightarrow 0} \sin \alpha=0$,

- $\lim _{\alpha \rightarrow 0} \cos \alpha=1$,

- $\dot{\theta}=\dot{\phi}=\dot{\psi}=0$.

This allows us to simplify Equation 2.2, which relates the body angular speeds to the Euler angles speeds, to the Identity matrix, i.e. $\dot{\phi}=p, \dot{\theta}=q$ and $\dot{\psi}=r$. Therefore, the equations of motion given at the end of Section 2.1 can be written as:

$$
\begin{aligned}
& \ddot{X}=(\sin \psi \sin \phi+\cos \psi \sin \theta \cos \phi) \frac{U_{1}}{m} \\
& \ddot{Y}=(-\cos \psi \sin \phi+\sin \psi \sin \theta \cos \phi) \frac{U_{1}}{m} \\
& \ddot{Z}=-g+(\cos \theta \cos \phi) \frac{U_{1}}{m} \\
& \ddot{\phi}=\frac{I_{y}-I_{z}}{I_{x}} \dot{\theta} \dot{\psi}-\frac{J_{T P}}{I_{x}} \dot{\theta} \Omega+\frac{U_{2}}{I_{x}} \\
& \ddot{\theta}=\frac{I_{z}-I_{x}}{I_{y}} \dot{\phi} \dot{\psi}+\frac{J_{T P}}{I_{y}} \dot{\phi} \Omega+\frac{U_{3}}{I_{y}} \\
& \ddot{\psi}=\frac{I_{x}-I_{y}}{I_{z}} \dot{\phi} \dot{\theta}+\frac{U_{4}}{I_{z}}
\end{aligned}
$$


with $\Omega=-\Omega_{1}+\Omega_{2}-\Omega_{3}+\Omega_{4}$ and

$$
\left[\begin{array}{c}
U_{1} \\
U_{2} \\
U_{3} \\
U_{4}
\end{array}\right]=\left[\begin{array}{c}
b\left(\Omega_{1}^{2}+\Omega_{2}^{2}+\Omega_{3}^{2}+\Omega_{4}^{2}\right) \\
l b\left(\Omega_{4}^{2}-\Omega_{2}^{2}\right) \\
l b\left(\Omega_{3}^{2}-\Omega_{1}^{2}\right) \\
d\left(-\Omega_{1}^{2}+\Omega_{2}^{2}-\Omega_{3}^{2}+\Omega_{4}^{2}\right)
\end{array}\right] .
$$

Since the controllers will be developed to stabilize four degrees-of-freedom of the prototype (pitch, roll, yaw and altitude), the first two equations can be left out. In fact, these two equations can be used as pitch and roll reference generators for the system to reach desired $X Y$ position.

While hovering, the vehicle also presents low rotational speed and all the motors have similar speed of rotation $(\Omega \approx 0)$ allowing the model to be further simplified to

$$
\begin{aligned}
\ddot{Z} & =-g+\frac{U_{1}}{m} \\
\ddot{\phi} & =\frac{U_{2}}{I_{x}} \\
\ddot{\theta} & =\frac{U_{3}}{I_{y}} \\
\ddot{\psi} & =\frac{U_{4}}{I_{z}} .
\end{aligned}
$$

One could replace $U_{1}$ to $U_{4}$ in this model with their relation to the propellers speed shown in Eq. 4.7 and then design controllers that already outputs a desired rotational speed. However, let us notice that when using $U_{1}$ to $U_{4}$ as inputs, the system becomes completely decoupled, facilitating the development of controllers.

Figure 23 shows a block diagram for the proposed approach. In short, based on the reference and on the states, the controller generates the output vector $U$, which is converted to squared motor speed by inverting Eq. (4.7) and written as

$$
\left[\begin{array}{l}
\Omega_{1}^{2} \\
\Omega_{2}^{2} \\
\Omega_{3}^{2} \\
\Omega_{4}^{2}
\end{array}\right]=\left[\begin{array}{cccc}
\frac{1}{4 b} & 0 & \frac{-1}{2 l b} & \frac{-1}{4 d} \\
\frac{1}{4 b} & \frac{-1}{2 l b} & 0 & \frac{1}{4 d} \\
\frac{1}{4 b} & 0 & \frac{1}{2 l b} & \frac{-1}{4 d} \\
\frac{1}{4 b} & \frac{1}{2 l b} & 0 & \frac{1}{4 d}
\end{array}\right]\left[\begin{array}{l}
U_{1} \\
U_{2} \\
U_{3} \\
U_{4}
\end{array}\right]
$$

and taking then its square root generates the speed reference to the BLDC motors, which directly control the quadcopter. 
Figure 23: Block diagram of the complete system.

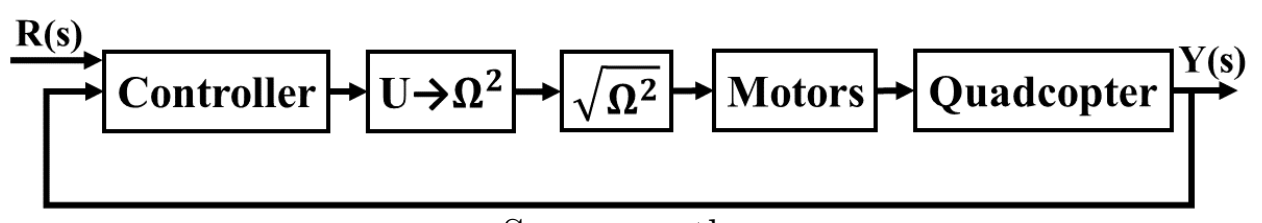

Source: author

\subsection{Controllability and Observability}

In short, a system is said to be controllable if exists a control law capable of transferring it from any initial state to another state in finite time; and it is said to be observable if, knowing the system model and its inputs, it is possible to determine a state based on output observations in finite time.

Consider an $n$-dimensional system written as

$$
\begin{aligned}
& \dot{\mathbf{x}}=\mathrm{Ax}+\mathrm{Bu} \\
& \mathbf{y}=\mathbf{C x},
\end{aligned}
$$

where $\mathbf{x}$ is the state vector, $\mathbf{u}$ is the control input, $\mathbf{y}$ is the system output and $\mathbf{A}, \mathbf{B}$ and $\mathbf{C}$ are constant matrices of appropriate sizes. Given the controllability matrix

$$
\mathbf{C}_{\mathbf{o}}=\left[\begin{array}{lllllllll}
\mathbf{B} & \vdots & \mathbf{A B} & \vdots & \mathbf{A}^{2} \mathbf{B} & \vdots & \cdots & \vdots & \mathbf{A}^{n-1} \mathbf{B}
\end{array}\right]
$$

a system is controllable if $\mathbf{C}_{\mathbf{o}}$ has rank $n$, i.e., if the vectors of $\mathbf{C}_{\mathbf{o}}$ are linearly independent. Similarly, the observability matrix is

$$
\mathbf{O}=\left[\begin{array}{c}
\mathrm{C} \\
\cdots \\
\mathrm{CA} \\
\cdots \\
\mathrm{CA}^{2} \\
\cdots \\
\vdots \\
\cdots \\
\mathrm{CA}^{n-1}
\end{array}\right]
$$

and a system is observable if $\mathbf{O}$ has rank $n$. 
Each state of the simplified quadcopter model can be written as

$$
\dot{\mathbf{x}}=\left[\begin{array}{ll}
0 & 1 \\
0 & 0
\end{array}\right] \mathbf{x}+\left[\begin{array}{c}
0 \\
1 / k
\end{array}\right] \mathbf{u},
$$

with $k$ the mass or inertia, depending on the equation. The controllability matrix is, then

$$
\mathbf{C}_{\mathbf{o}}=\left[\begin{array}{lll}
\mathbf{B} & \vdots & \mathbf{A B}
\end{array}\right]=\left[\begin{array}{cc}
0 & 1 / k \\
1 / k & 0
\end{array}\right],
$$

which has rank 2, proving the system to be controllable. Its observability matrix is

$$
\mathbf{O}=\left[\begin{array}{c}
\mathbf{C} \\
\cdots \\
\mathbf{C A}
\end{array}\right]=\left[\begin{array}{ll}
1 & 0 \\
0 & 1
\end{array}\right],
$$

also a rank 2 matrix, an observable system.

\subsection{Tests Procedure}

A simulation environment was developed at Matlab/Simulink ${ }^{\circledR}$ based on Figure 23 and using the nonlinear model equations described at Section 2.1. The first-order model of the motors were included as well.

Controllers were all implemented as if they were embedded on a microcontroller: they were discretized, used with fixed sampling time and written in code using the Matlab Function block. Simulated noise were added to the system output based on initial tests on the sensors, being considered a Gaussian process with null mean and variance of $10^{-5}$.

To standardize the simulation and application procedures, every controller was subject to the same sampling time $(100 \mathrm{~Hz})$ and reference signal pattern, described in the next items:

- hover at $0.1 \mathrm{~m}$;

- at $10 \mathrm{~s}$, a $0.2 \mathrm{rad}$ reference is set to $\phi$;

- at $20 \mathrm{~s}, \mathrm{a}-0.2 \mathrm{rad}$ reference is set to $\theta$;

- at $30 \mathrm{~s}, \psi$ gets a $0.25 \mathrm{rad}$ step reference;

- at $40 \mathrm{~s}, 41 \mathrm{~s}$ and $42 \mathrm{~s}, \phi, \theta$ and $\psi$ return to zero, respectively;

- at $50 \mathrm{~s}$, a $10 \mathrm{~s}$ step reference of $0.2 \mathrm{~m}$ is set to altitude. 


\subsection{PID Controllers}

Section 3.1 described three PID types: ISA-form, output derivative $(\mathrm{PI}+\mathrm{D})$ and derivative-free. Initial simulations considered all these controllers but, based on the better results presented, only the last two had the discretized version implemented and only these are described in this text.

As pitch and roll were assumed to have equal dynamics in the modeling process, just three PIDs have to be designed: altitude, yaw and pitch-roll. Based on the simplified model shown in Section 4.1, it is known that using a PD controller would be enough to stabilize the system, because its transfer function is a double integrator. Inserting the integral term is interesting for guaranteeing null steady-state error even with disturbances.

The controller parameters are tuned based on the following transfer functions and using the Matlab PIDTuner function.

$$
Z(s)=\frac{2.222}{s^{2}} \quad \phi(s)=\frac{443.1}{s^{2}} \quad \theta(s)=\frac{443.1}{s^{2}} \quad \psi(s)=\frac{227.1}{s^{2}}
$$

One usual procedure for choosing the PID parameters is making one controller zero cancel a stable system pole, and then choosing the rest of the parameters according to performance specifications, allocating the dominant closed loop poles. However, this procedure cannot guarantee the desired specifications for a double integral system due to the presence of other closed loop zeros and poles. The PIDTuner function offers graphic interface for online tunning, allowing the user to weight between a faster or slower system, and between a more robust or aggressive one.

The parameters were manually tuned so that the system presents overshoot close to $15 \%$ and settling time close to $3 \mathrm{~s}$. Table 3 presents the chosen PID parameters to be used in every PID implementation.

Table 3: PID parameters

\begin{tabular}{cccc} 
Parameter & Altitude & Pitch/Roll & Yaw \\
\hline$K_{c}$ & 8.3556 & 0.3000 & 0.1500 \\
$T_{I}$ & 2.1361 & 1.0000 & 1.5000 \\
$T_{D}$ & 0.5245 & 0.1500 & 0.1500 \\
$N$ & 150 & 30 & 30 \\
\hline
\end{tabular}

To highlight the difference between both PID implementations, the results are presented in two groups: with and without simulated noise included. The main results are presented next, with extra results attached in Appendix A.1. 
In order to highlight the benefits from using the derivative-free PID proposed, the simulation results are presented in two figures. The first, Figure 24, is obtained without measurement noise, while the second, Figure 25, takes it into account. Inside each figure, states and control effort for the output derivative PID are depicted in items (a) and (b), and for the derivative-free in $(\mathrm{c})$ and $(\mathrm{d})$. Implementation results are presented in the same order in Figure 26.

Comparing the results produced by both PID implementations, output derivative and derivative-free, they presented similar simulation response when noise was not included, fact that is expected since feedbacking a perfect velocity signal and deriving a perfect position signal will produce equal values. The effectiveness of the derivative-free implementation stands out when sensor noise is considered, since its control effort is less noisy when compared to the output derivative method, as seen in Figure 25. However, both implementation results presented in Figure 26 show similar response, with much more oscillations than in simulation and higher overshoot in the angular positioning. Altitude control presents smaller fluctuation when using the derivative-free form, although both behaves similarly. 
Figure 24: Simulation results for the discretized PID controllers without noise.

(a) Controlled states with output derivative PID.

(b) Control effort with output derivative PID.
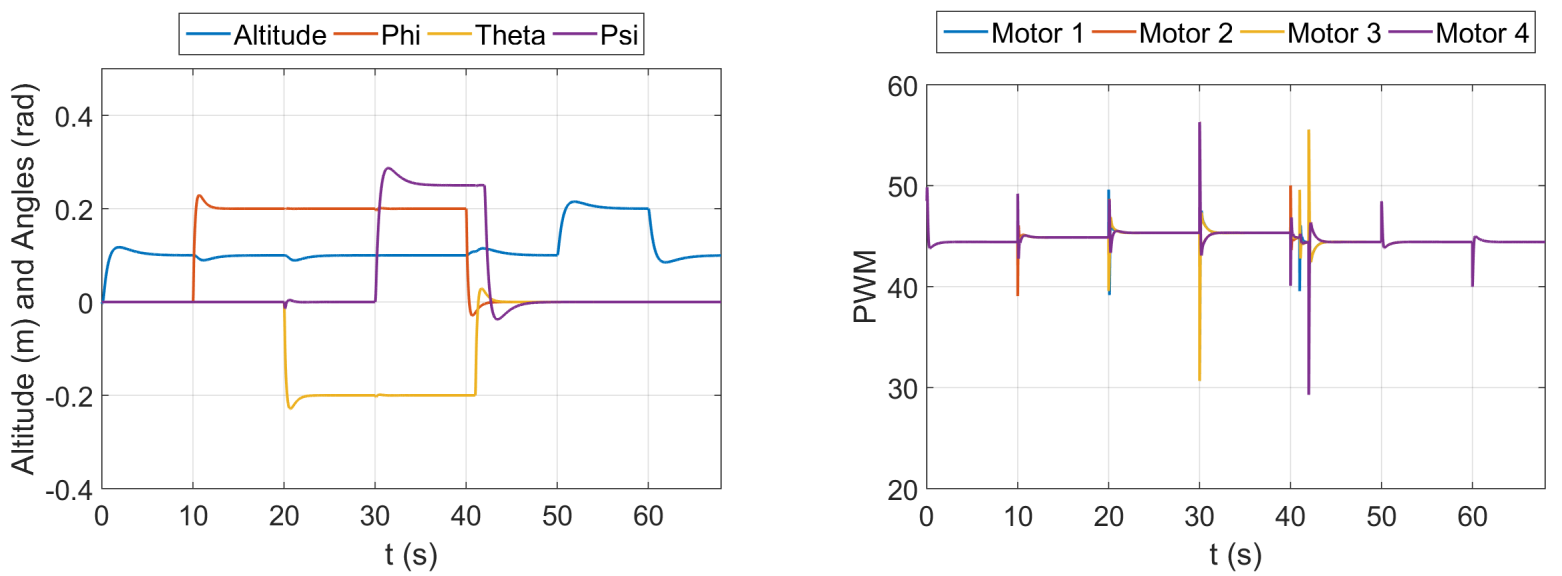

(c) Controlled states with derivative-free PID.

(d) Control effort with derivative-free PID.
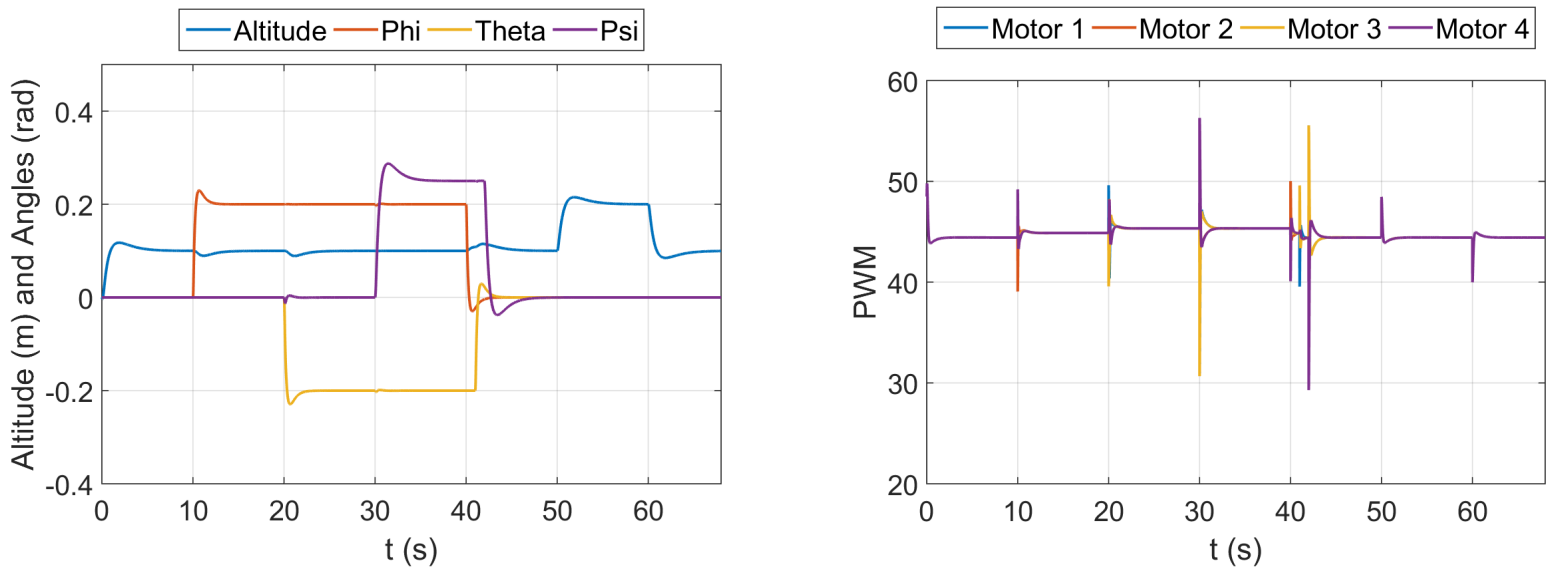

Source: author 
Figure 25: Simulation results for the PID discretized controllers with noise.

(a) Controlled states with output derivative PID.

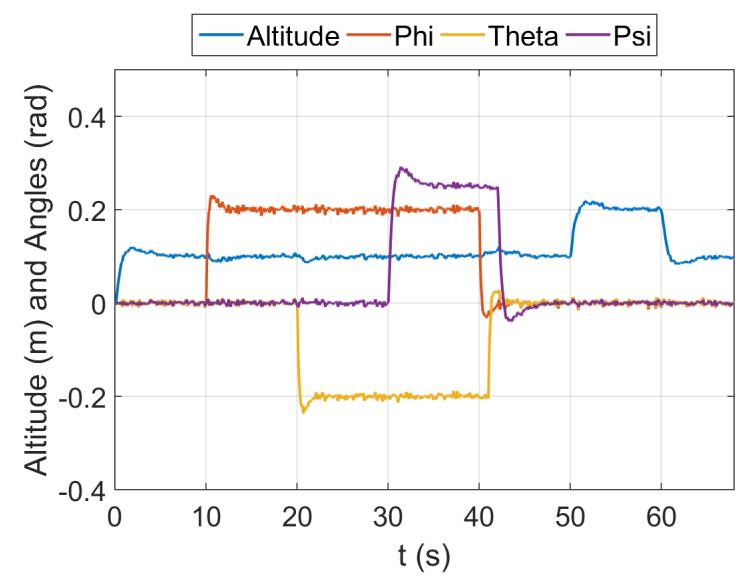

(c) Controlled states with derivative-free PID.

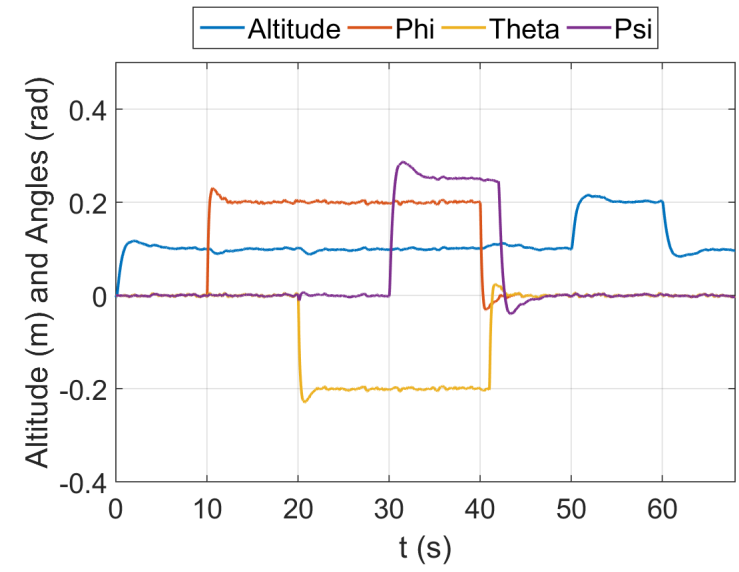

(b) Control effort with output derivative PID.

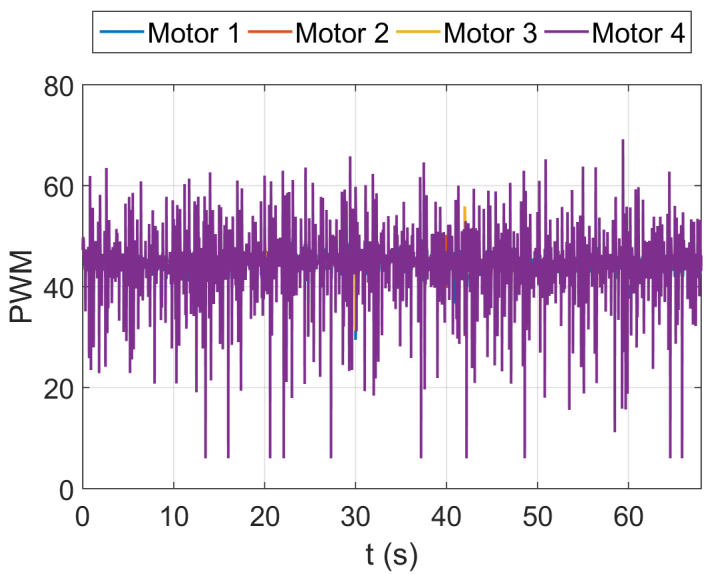

(d) Control effort with derivative-free PID.

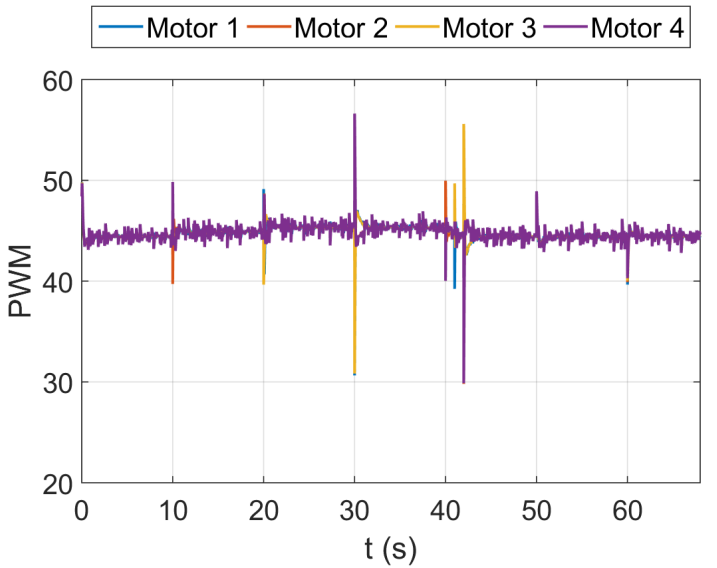

Source: author 
Figure 26: Practical results for the PID.

(a) Controlled states with output derivative PID.

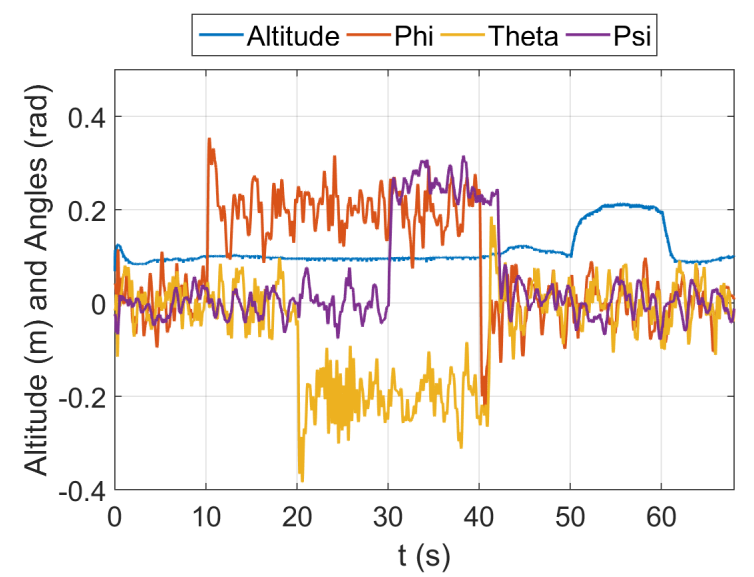

(c) Controlled states with derivative-free PID.

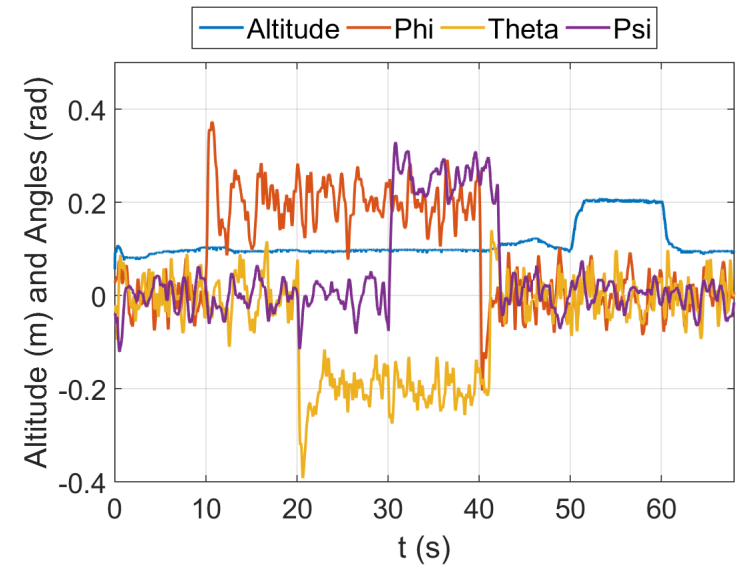

(b) Control effort with output derivative PID.

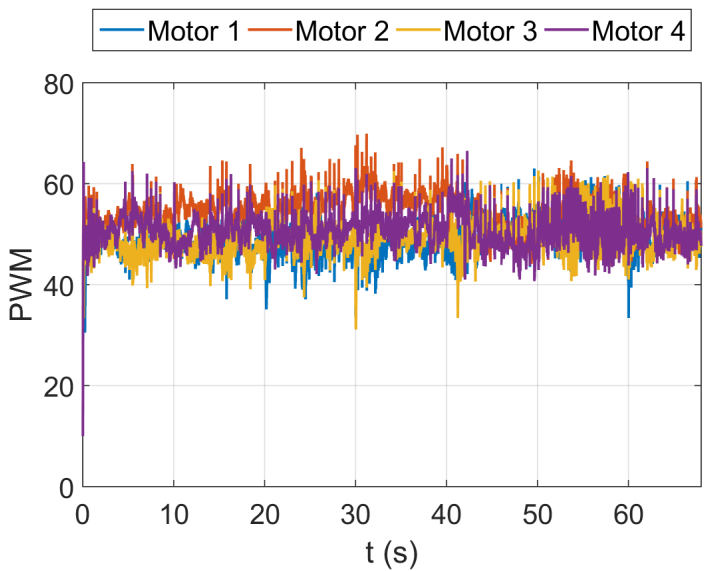

(d) Control effort with derivative-free PID.

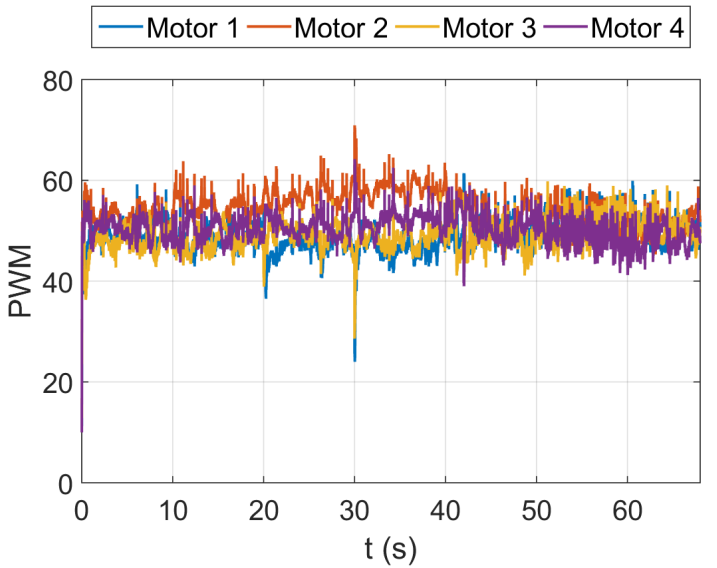

Source: author 


\subsection{LQR Controller}

For simplicity, Matlab lqr function was used to obtain the feedback gain matrix. Since the system is decoupled, this function can be used once to produce an also decoupled gain matrix.

Organizing the state vector as $\mathbf{x}=\left[\begin{array}{llllllll}z & \dot{z} & \phi & \dot{\phi} & \theta & \dot{\theta} & \psi & \dot{\psi}\end{array}\right]^{T}$, matrices $\mathbf{A}$ and $\mathbf{B}$ are

$$
\mathbf{A}=\left[\begin{array}{llllllll}
0 & 1 & 0 & 0 & 0 & 0 & 0 & 0 \\
0 & 0 & 0 & 0 & 0 & 0 & 0 & 0 \\
0 & 0 & 0 & 1 & 0 & 0 & 0 & 0 \\
0 & 0 & 0 & 0 & 0 & 0 & 0 & 0 \\
0 & 0 & 0 & 0 & 0 & 1 & 0 & 0 \\
0 & 0 & 0 & 0 & 0 & 0 & 0 & 0 \\
0 & 0 & 0 & 0 & 0 & 0 & 0 & 1 \\
0 & 0 & 0 & 0 & 0 & 0 & 0 & 0
\end{array}\right], \mathbf{B}=\left[\begin{array}{cccc}
0 & 0 & 0 & 0 \\
2.2222 & 0 & 0 & 0 \\
0 & 0 & 0 & 0 \\
0 & 443.0660 & 0 & 0 \\
0 & 0 & 0 & 0 \\
0 & 0 & 443.0660 & 0 \\
0 & 0 & 0 & 0 \\
0 & 0 & 0 & 227.0663
\end{array}\right],
$$

with which, after inserting integrators (as described in Section 3.2), the LQR is designed. By manually tuning LQR matrices $\mathbf{Q}$ and $\mathbf{R}$ to $\mathbf{R}=\operatorname{diag}\left(\left[\begin{array}{llll}0.1 & 1 & 1 & 1\end{array}\right]\right)$ and $\mathbf{Q}=\operatorname{diag}\left(\left[\begin{array}{llllllllllll}0.5 & 10^{-5} & 0.1 & 10^{-5} & 0.1 & 10^{-5} & 0.01 & 10^{-5} & 1 & 1 & 1 & 0.01\end{array}\right]\right)$, the following $\mathbf{K}_{a u g}$ was obtained:

$$
\begin{aligned}
\mathbf{K} & =\left[\begin{array}{cccccccc}
4.150 & 1.933 & 0 & 0 & 0 & 0 & 0 & 0 \\
0 & 0 & 0.437 & 0.045 & 0 & 0 & 0 & 0 \\
0 & 0 & 0 & 0 & 0.437 & 0.045 & 0 & 0 \\
0 & 0 & 0 & 0 & 0 & 0 & 0.131 & 0.035
\end{array}\right] \\
\mathbf{K}_{I} & =\left[\begin{array}{cccc}
3.162 & 0 & 0 & 0 \\
0 & 1.000 & 0 & 0 \\
0 & 0 & 1.000 & 0 \\
0 & 0 & 0 & 0.100
\end{array}\right] \\
\mathbf{K}_{\text {aug }}= & {\left[\mathbf{K}-\mathbf{K}_{I}\right] . }
\end{aligned}
$$

The main characteristic aimed at when tunning this controller was limiting the dependence on the derivative gains, usually a very noisy signal. This was achieved by setting matrix $Q$ respective values to $10^{-5}$. Then, the spots corresponding to proportional and integral gains were tuned. 
Simulation results are presented in Figure 27a with the controlled states plot and in Figure 27b with the control effort, in which the controller is already discretized and noise is included. Figures $27 \mathrm{c}$ and $27 \mathrm{~d}$ present the controlled states and the control efforts, respectively, resultants from tests on the prototype.

LQR augmented with integrators is the controller that presented the smallest oscillation amplitude around the set-point and a closer-to-simulation practical results.

Figure 27: Simulation and test results for the discretized LQR controllers with noise.

(a) Controlled states.

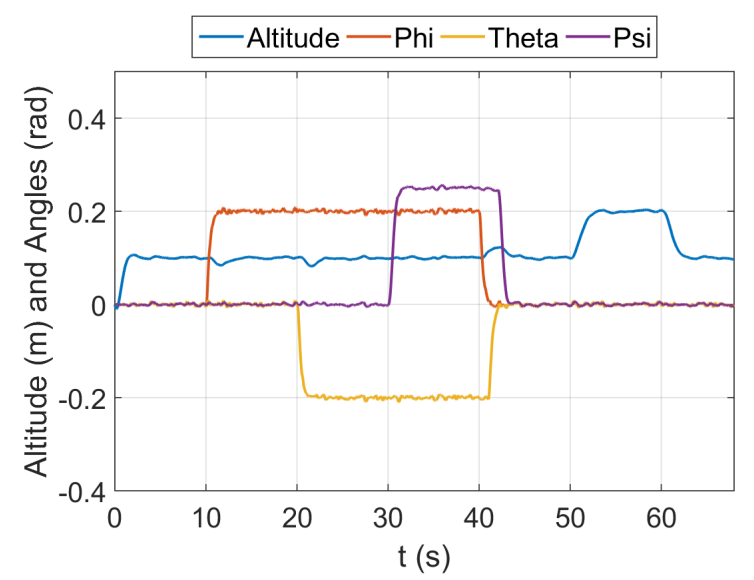

(c) Controlled states.

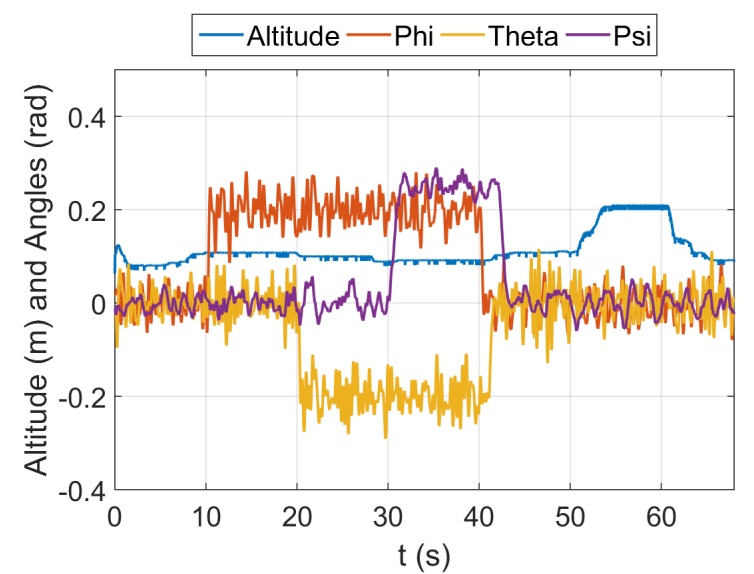

(b) Control effort.

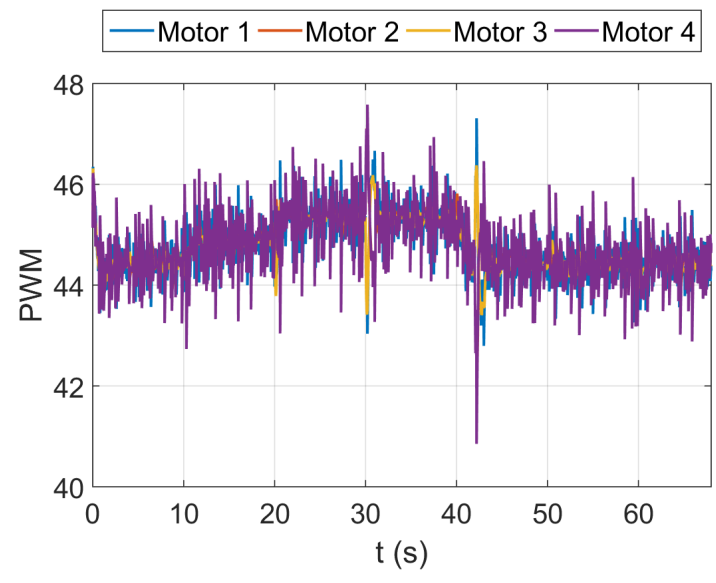

(d) Control effort.

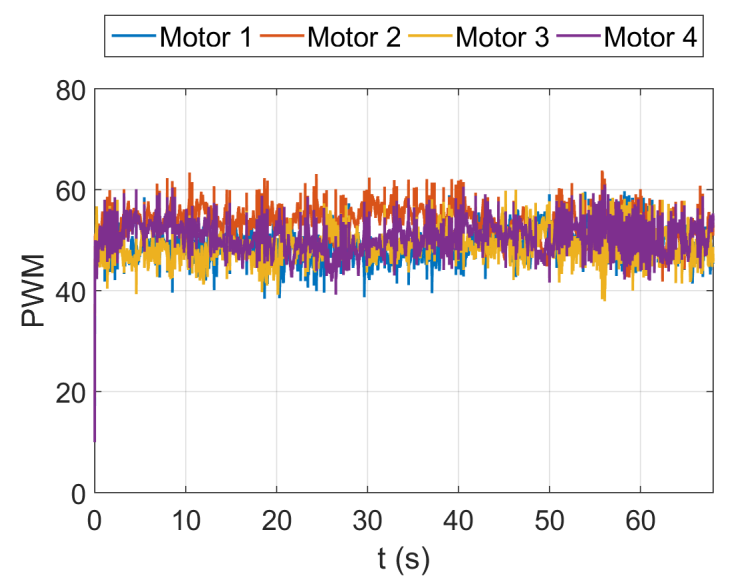

Source: author

\subsection{Sliding Mode Controllers}

The use of a nonlinear controller with guaranteed robustness margins is proposed in this section. The complete model shows the system is directly influenced by five parameters: $m, I_{x}, I_{y}, I_{z}$ and $J_{T P}$. However, by assuming that $J_{T P}$ is too small when 
compared to the other parameters and that the system will be hovering most part of the time, $J_{T P}$ can be neglected. Each parameter is assumed to vary $\pm 10 \%$ around the nominal value; maximum and minimum admissible values are presented in Table 4 .

Table 4: Admissible Parameter Values

\begin{tabular}{cccc} 
Parameter & Min & Max & Unit \\
\hline$m$ & 0.405 & 0.495 & $\mathrm{~kg}$ \\
$I_{x}$ & 2.031 & 2.483 & $10^{-3} \mathrm{~kg} \cdot \mathrm{m}^{2}$ \\
$I_{y}$ & 2.031 & 2.483 & $10^{-3} \mathrm{~kg} \cdot \mathrm{m}^{2}$ \\
$I_{z}$ & 3.964 & 4.844 & $10^{-3} \mathrm{~kg} \cdot \mathrm{m}^{2}$ \\
\hline
\end{tabular}

First, it is presented the development of the altitude controller, followed by pitch, roll and yaw, which are written together.

\subsubsection{Altitude Controller}

The equation that describes the altitude dynamics is

$$
\ddot{Z}=-g+(\cos \theta \cos \phi) \frac{U_{1}}{m}=-g+b U_{1},
$$

with $b=(\cos \theta \cos \phi) / m$. The gravity is assumed to be known with precision and equal to $9.83 \mathrm{~m} / \mathrm{s}^{2}$, the mass may vary $10 \%$ from nominal one while $\phi$ and $\theta$ are feedback terms.

Since the exact value of $b$ is unknown, an approximation is required, and two approaches can be considered: one is defining maximum ranges for $\phi$ and $\theta$ and then having a fixed $\hat{b}$, while the other option is using the feedback angles to update this approximation to a more exact value. Indeed, the latter option is better, because depending on the range of the values chosen for both angles, the controller may act too aggressively for small angles or too sluggish for wider angles.

Thus, the following equation is valid

$$
0<b_{\min }<b<b_{\max }
$$

i.e.,

$$
0<\frac{\cos \theta \cos \phi}{m_{\max }}<b<\frac{\cos \theta \cos \phi}{m_{\min }},
$$

and $\hat{b}$ can be chosen such that

$$
\hat{b}=\left(b_{\min } b_{\max }\right)^{\frac{1}{2}}=\cos \theta \cos \phi\left(\frac{1}{m_{\min } m_{\max }}\right)^{\frac{1}{2}}
$$


and

$$
\beta=\left(b_{\max } / b_{\min }\right)^{\frac{1}{2}}=\left(m_{\max } / m_{\min }\right)^{\frac{1}{2}}
$$

Defining a sliding surface for this subsystem as

$$
s_{Z}=\dot{\tilde{Z}}+\lambda_{Z} \tilde{Z}
$$

its derivative is developed as

$$
\begin{aligned}
\dot{s}_{Z} & =\ddot{\tilde{Z}}+\lambda_{Z} \dot{\tilde{Z}} \\
& =\ddot{Z}-\ddot{Z}_{d}+\lambda_{Z} \dot{\tilde{Z}} \\
& =-g+b U_{1}-\ddot{Z}_{d}+\lambda_{Z} \dot{\tilde{Z}} .
\end{aligned}
$$

By choosing the controller such that

$$
\begin{aligned}
& \hat{U}_{1}=g+\ddot{Z}_{d}-\lambda_{Z} \dot{\tilde{Z}} \\
& U_{1}=\frac{1}{\hat{b}}\left[\hat{U}_{1}-K_{Z} \operatorname{sat}\left(s_{Z} / \Phi_{Z}\right)\right],
\end{aligned}
$$

where $K_{Z}$ must satisfy the sliding condition, what is achieved by any $K_{Z}$ that satisfies the following condition:

$$
K_{Z} \geq \beta \eta+(\beta-1)\left|\hat{U}_{1}\right|
$$

\subsubsection{Attitude Controllers}

Since pitch, roll and yaw dynamics are very similar, their controllers will be developed together. Their equations are:

$$
\begin{aligned}
& \ddot{\phi}=\frac{I_{y}-I_{z}}{I_{x}} \dot{\theta} \dot{\psi}+\frac{U_{2}}{I_{x}}=\underbrace{f_{1_{\phi}} \dot{\theta} \dot{\psi}}_{f_{\phi}}+b_{\phi} U_{2}, \\
& \ddot{\theta}=\frac{I_{z}-I_{x}}{I_{y}} \dot{\phi} \dot{\psi}+\frac{U_{3}}{I_{y}}=\underbrace{f_{1_{\theta}} \dot{\phi} \dot{\psi}}_{f_{\theta}}+b_{\theta} U_{3}, \\
& \ddot{\psi}=\frac{I_{x}-I_{y}}{I_{z}} \dot{\phi} \dot{\theta}+\frac{U_{4}}{I_{z}}=\underbrace{f_{1_{\psi}} \dot{\phi} \dot{\theta}}_{f_{\psi}}+b_{\psi} U_{4}
\end{aligned}
$$


in which $I_{x}, I_{y}$ and $I_{z}$ may vary $10 \%$ from the nominal ones. Defining the sliding surfaces as

$$
\begin{aligned}
& s_{\phi}=\dot{\tilde{\phi}}+\lambda_{\phi} \tilde{\phi} \\
& s_{\theta}=\dot{\tilde{\theta}}+\lambda_{\theta} \tilde{\theta} \\
& s_{\psi}=\dot{\tilde{\psi}}+\lambda_{\psi} \tilde{\psi},
\end{aligned}
$$

their derivatives are

$$
\begin{aligned}
\dot{s}_{\phi} & =\ddot{\tilde{\phi}}+\lambda_{\phi} \dot{\tilde{\phi}} \\
& =\ddot{\phi}-\ddot{\phi}_{d}+\lambda_{\phi} \dot{\tilde{\phi}} \\
& =f_{\phi}+b_{\phi} U_{2}-\ddot{\phi}_{d}+\lambda_{\phi} \dot{\tilde{\phi}} ; \\
\dot{s}_{\theta} & =\ddot{\tilde{\theta}}+\lambda_{\theta} \dot{\tilde{\theta}} \\
& =\ddot{\theta}-\ddot{\theta}_{d}+\lambda_{\theta} \dot{\tilde{\theta}} \\
& =f_{\theta}+b_{\theta} U_{3}-\ddot{\theta}_{d}+\lambda_{\theta} \dot{\tilde{\theta}} \\
\dot{s}_{\psi} & =\ddot{\tilde{\psi}}+\lambda_{\psi} \dot{\tilde{\psi}} \\
& =\ddot{\psi}-\ddot{\psi_{d}}+\lambda_{\psi} \dot{\tilde{\psi}} \\
& =f_{\psi}+b_{\psi} U_{4}-\ddot{\psi}_{d}+\lambda_{\psi} \dot{\tilde{\psi}} .
\end{aligned}
$$

Then, the controllers can be chosen as

$$
\begin{aligned}
\hat{U}_{2} & =-\hat{f}_{\phi}+\ddot{\phi}_{d}-\lambda_{\phi} \dot{\tilde{\phi}}, \\
U_{2} & =\frac{1}{\hat{b}_{\phi}}\left[\hat{U}_{2}-K_{\phi} \operatorname{sat}\left(s_{\phi} / \Phi_{\phi}\right)\right] ; \\
\hat{U}_{3} & =-\hat{f}_{\theta}+\ddot{\theta}_{d}-\lambda_{\theta} \dot{\tilde{\theta}}, \\
U_{3} & =\frac{1}{\hat{b}_{\theta}}\left[\hat{U}_{3}-K_{\theta} \operatorname{sat}\left(s_{\theta} / \Phi_{\theta}\right)\right] ; \\
\hat{U}_{4} & =-\hat{f}_{\psi}+\ddot{\psi}_{d}-\lambda_{\psi} \dot{\tilde{\psi}} \\
U_{4} & =\frac{1}{\hat{b}_{\psi}}\left[\hat{U}_{4}-K_{\psi} \operatorname{sat}\left(s_{\psi} / \Phi_{\psi}\right)\right],
\end{aligned}
$$

with $\hat{b}=\left(b_{\min } b_{\max }\right)^{\frac{1}{2}}$ and $\hat{f}=\frac{f_{\max }-f_{\min }}{2}+f_{\min }$. 
In order to evaluate the sliding condition, it is developed the following error analysis:

$$
\begin{aligned}
F_{\phi} & =\left|\hat{f}_{\phi}-f_{\phi}\right| \\
& =\left|\left(\hat{f}_{1_{\phi}}-f_{1_{\phi}}\right) \dot{\theta} \dot{\psi}\right| \\
& =\left|F_{1_{\phi}} \dot{\theta} \dot{\psi}\right| \\
F_{\theta} & =\left|\hat{f}_{\theta}-f_{\theta}\right| \\
& =\left|\left(\hat{f}_{1_{\theta}}-f_{1_{\theta}}\right) \dot{\phi} \dot{\psi}\right| \\
& =\left|F_{1_{\theta}} \dot{\phi} \dot{\psi}\right| \\
F_{\psi} & =\left|\hat{f}_{\psi}-f_{\psi}\right| \\
& =\left|\left(\hat{f}_{1_{\psi}}-f_{1_{\psi}}\right) \dot{\phi} \dot{\theta}\right| \\
& =\left|F_{1_{\psi}} \dot{\phi} \dot{\theta}\right|,
\end{aligned}
$$

and then the sliding condition is satisfied if the following are satisfied:

$$
\begin{aligned}
& K_{\phi} \geq \beta_{\phi}\left(F_{\phi}+\eta_{\phi}\right)+\left(\beta_{\phi}-1\right)\left|\hat{U}_{2}\right|, \\
& K_{\theta} \geq \beta_{\theta}\left(F_{\theta}+\eta_{\theta}\right)+\left(\beta_{\theta}-1\right)\left|\hat{U}_{3}\right|, \\
& K_{\psi} \geq \beta_{\psi}\left(F_{\psi}+\eta_{\psi}\right)+\left(\beta_{\psi}-1\right)\left|\hat{U}_{4}\right|,
\end{aligned}
$$

with $\beta=\left(b_{\max } / b_{\min }\right)^{\frac{1}{2}}$.

\subsubsection{Parameters and Specifications}

The next step is choosing the values of each $\eta, \lambda$ and $\Phi$, which have direct influences on the results. The chosen values are all stated in Table 5.

Table 5: Sliding Mode Controller Parameters

\begin{tabular}{cc||cc||cc} 
Parameter & Value & Parameter & Value & Parameter & Value \\
\hline$\eta_{Z}$ & 4 & $\lambda_{Z}$ & 4 & $\Phi_{Z}$ & 0.8 \\
$\eta_{\phi}$ & 4 & $\lambda_{\phi}$ & 4 & $\Phi_{\phi}$ & 0.1 \\
$\eta_{\theta}$ & 4 & $\lambda_{\theta}$ & 4 & $\Phi_{\theta}$ & 0.1 \\
$\eta_{\psi}$ & 6 & $\lambda_{\psi}$ & 3 & $\Phi_{\psi}$ & 0.3 \\
\hline
\end{tabular}

The time required for the system to reach the surface is given by

$$
t_{r} \leq \frac{|s(0)|}{\eta}
$$

which means that if the quadcopter initial condition is hovering $1 \mathrm{~m}$ below zero, then it 
will take less than 1 s to reach $s=0$. The same is valid for pitch and roll, in which if the quadcopter begins at 1rad it will take less than 1s to reach the surface, while this time is 0.5 s for yaw.

After the surface is reached, the desired state is reached with time constant equal to $1 / \lambda$, i.e., $0.333 \mathrm{~s}$ for $\psi$ and $0.25 \mathrm{~s}$ for the remaining. Simulations showing these characteristics are presented in Appendix A.2.

At last, each state has a guaranteed error bound smaller than $\Phi / \lambda$ which, applied to this quadcopter, provides maximum error of $20 \mathrm{~cm}$ for altitude, $5.7 \mathrm{deg}$ for yaw and $1.4 \mathrm{deg}$ for pitch and roll.

\subsubsection{Reference filtering and its derivatives}

Since this nonlinear controller uses the second derivative of the reference, some filtering is advisable, mainly for smoothing a step-input and limiting its derivatives.

Firstly, the reference is filtered by a second order system with natural frequency of $10 \mathrm{rad} / \mathrm{s}$ and unitary damping coefficient, which is written as

$$
\text { Filter }=\frac{10^{2}}{s^{2}+20 s+10^{2}}
$$

Even though this might seem enough, it is necessary to filter the high-frequency part of its derivatives. Thus, the same filter is used to the first and second derivatives, with their equation being

$$
\text { Derivative }=\frac{100^{2} s}{s^{2}+200 s+100^{2}}
$$

These filters and derivatives are the only part of the Sliding Mode Controller that need discretization to be embedded in the development board; the output derivative is already provided by the sensors.

\subsubsection{Simulation Results}

Figure 28 presents the simulation results for the quadcopter with sliding mode controller including measurement noise. Noiseless simulations are attached in Appendix A.2 due to the similarity with the figures presented here. Figures presenting the surface convergence on each controller are also in Appendix A.2. 


\subsubsection{Robustness Simulation}

The development of this controller was based on the decoupled model of the quadcopter, in which the inputs are the generalized variables $U_{1}$ to $U_{4}$. This guarantees robustness in no other parameter besides the ones on this decoupled model (mass and inertias), i.e., all the parameters that translate the generalized inputs to each motors PWM signal (thrust and torque of each propeller and the relation of speed and PWM of each motor) have no guarantees.

Let us rewrite the nonlinear equation of motion related to the angle $\phi$ replacing $U_{2}$ with a more realistic approach:

$$
\ddot{\phi}=\frac{I_{y}-I_{z}}{I_{x}} \dot{\theta} \dot{\psi}-\frac{J_{T P}}{I_{x}} \dot{\theta} \Omega+\frac{l}{I_{x}}\left(b_{4} \Omega_{4}^{2}-b_{2} \Omega_{2}^{2}\right),
$$

in which $b_{2}$ and $b_{4}$ are the thrust coefficients of propellers 2 and 4 , respectively. Figure 29a shows the simulation results when using $b_{2} 6 \%$ smaller than nominal and $b_{4}$ unchanged.

Additionally, as the motors are not perfectly equal, they present slightly different transfer function relating PWM signal to rotational speed. Figure $29 \mathrm{~b}$ depicts the simulation result considering motor number 1 to be $3 \%$ slower than the others. As Figure 29 shows, this controller is unable to stabilize the system when motors and/or propellers differ among each other. Therefore, no practical results are presented in this text.

Before varying different parameters in simulation, this controller was uploaded to the Arduino and some tests were performed. Then, after concluding it would not control the quadcopter, some parameters were changed in simulation in order to understand the possible causes: the controller is indeed robust to variations in mass and inertias, but not to variations from motors and propellers.

\subsection{Integrative Sliding Mode Controller with LQR}

Aiming on developing a controller based on a coupled model, the simplified model presented in Section 4.1 was not used. Instead, $U_{1}$ to $U_{4}$ were replaced by their relation with the motors squared speed, and these by their linear relation to the signal sent to the 
Figure 28: Controlled states with Sliding Mode Controllers

(a) States with noise.

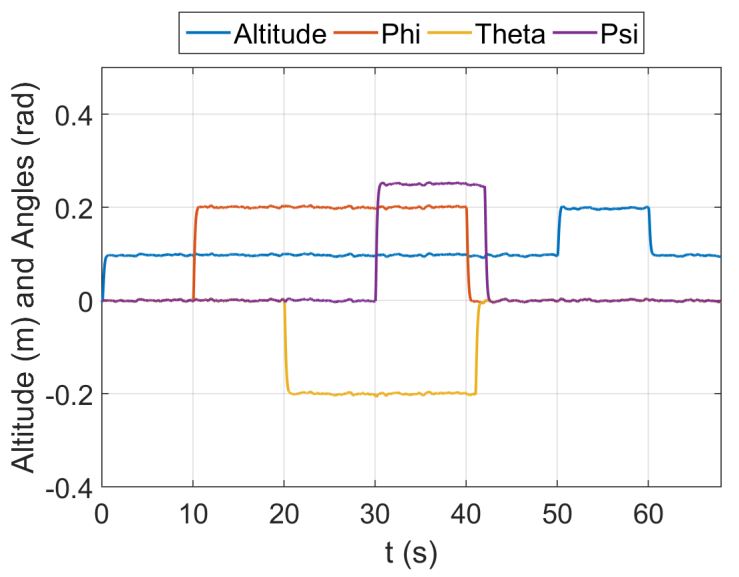

(b) Control effort with noise.

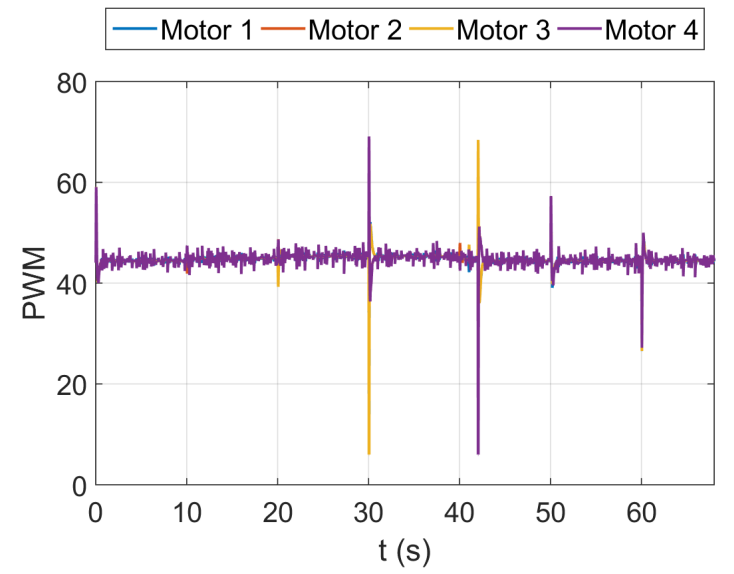

Source: author

Figure 29: Sliding Mode Controllers robustness simulations.

(a) $b_{2} 6 \%$ smaller.

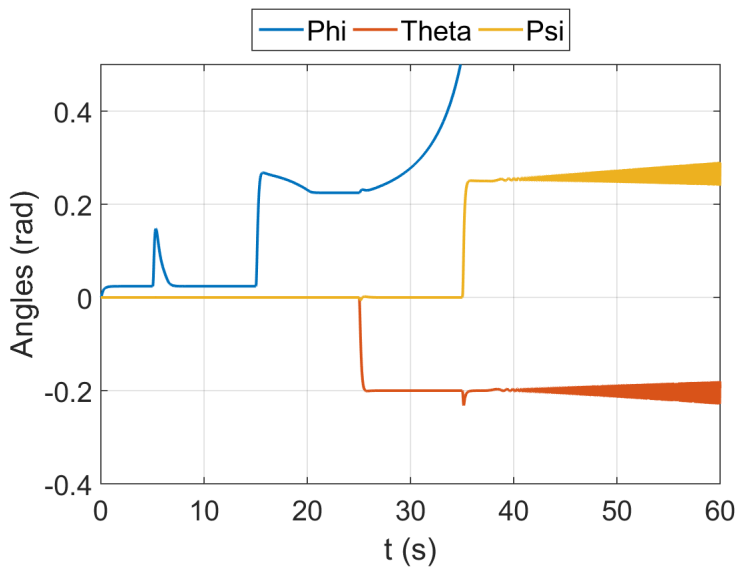

(b) motor $13 \%$ slower than the others.

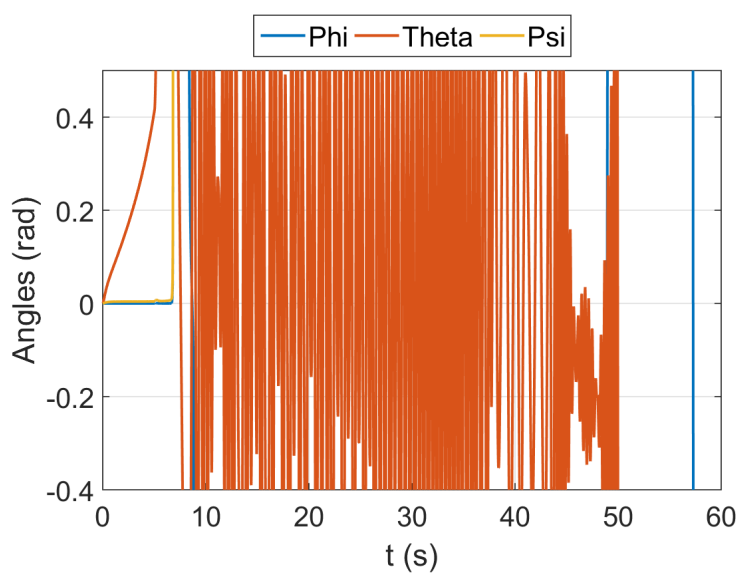

Source: author

ESC. The new equations of motion are

$$
\begin{aligned}
& \ddot{Z}=-g+(\cos \theta \cos \phi) \frac{a^{2} b}{m}\left(M_{1}^{2}+M_{2}^{2}+M_{3}^{2}+M_{4}^{2}\right) \\
& \ddot{\phi}=\frac{I_{y}-I_{z}}{I_{x}} \dot{\theta} \dot{\psi}+\frac{a^{2} l b}{I_{x}}\left(M_{4}^{2}-M_{2}^{2}\right) \\
& \ddot{\theta}=\frac{I_{z}-I_{x}}{I_{y}} \dot{\phi} \dot{\psi}+\frac{a^{2} l b}{I_{y}}\left(M_{3}^{2}-M_{1}^{2}\right) \\
& \ddot{\psi}=\frac{I_{x}-I_{y}}{I_{z}} \dot{\phi} \dot{\theta}+\frac{a^{2} d}{I_{z}}\left(-M_{1}^{2}+M_{2}^{2}-M_{3}^{2}+M_{4}^{2}\right),
\end{aligned}
$$


in which $M_{\alpha}$ is the signal sent to motor $\alpha$ and $a$ is the constant that relates this signal to the speed of rotation. These equations can be written in the form

$$
\dot{\mathbf{x}}(t)=\mathbf{A x}(t)+\mathbf{B u}(t)+\mathbf{B D} \xi(t, x)
$$

by using the same state vector as in the LQR controller, $\mathbf{x}=\left[\begin{array}{llllllll}z & \dot{z} & \phi & \dot{\phi} & \theta & \dot{\theta} & \psi & \dot{\psi}\end{array}\right]^{T}$, but changing the control one to $\mathbf{u}=\left[\begin{array}{llll}M_{1}^{2} & M_{2}^{2} & M_{3}^{2} & M_{4}^{2}\end{array}\right]^{T}$. Thus, the matrices are as follows

$$
\mathbf{A}=\left[\begin{array}{llllllll}
0 & 1 & 0 & 0 & 0 & 0 & 0 & 0 \\
0 & 0 & 0 & 0 & 0 & 0 & 0 & 0 \\
0 & 0 & 0 & 1 & 0 & 0 & 0 & 0 \\
0 & 0 & 0 & 0 & 0 & 0 & 0 & 0 \\
0 & 0 & 0 & 0 & 0 & 1 & 0 & 0 \\
0 & 0 & 0 & 0 & 0 & 0 & 0 & 0 \\
0 & 0 & 0 & 0 & 0 & 0 & 0 & 1 \\
0 & 0 & 0 & 0 & 0 & 0 & 0 & 0
\end{array}\right], \mathbf{B}=\left[\begin{array}{cccc}
0 & 0 & 0 & 0 \\
b / m & b / m & b / m & b / m \\
0 & 0 & 0 & 0 \\
0 & -a^{2} l b / I_{x} & 0 & a^{2} l b / I_{x} \\
0 & 0 & 0 & 0 \\
-a^{2} l b / I_{y} & 0 & a^{2} l b / I_{y} & 0 \\
0 & 0 & 0 & 0 \\
-a^{2} d / I_{z} & a^{2} d / I_{z} & -a^{2} d / I_{z} & a^{2} d / I_{z}
\end{array}\right]
$$

$$
\mathbf{B D} \xi(t, x)=\left[\begin{array}{c}
0 \\
-g \\
0 \\
\left(I_{y}-I_{z}\right) \dot{\theta} \dot{\psi} / I_{x} \\
0 \\
\left(I_{z}-I_{x}\right) \dot{\phi} \dot{\psi} / I_{y} \\
0 \\
\left(I_{x}-I_{y}\right) \dot{\phi} \dot{\theta} / I_{z}
\end{array}\right]
$$

Augmenting this system with integrators, as explained in Section 3.2 and then following the procedure from Section 3.4, the controller that combines Integral Sliding Mode with LQR is ready to be tuned.

By manually tuning LQR matrices $\mathbf{Q}$ and $\mathbf{R}$ to $\mathbf{R}=\operatorname{diag}\left(\left[\begin{array}{llll}10^{-3} & 10^{-3} & 10^{-3} & 10^{-3}\end{array}\right]\right)$ and $\mathbf{Q}=\operatorname{diag}\left(\left[\begin{array}{llllllllllll}10^{6} & 10^{4} & 10^{4} & 10^{2} & 10^{4} & 10^{2} & 10^{4} & 10^{1} & 10^{7} & 10^{4} & 10^{4} & 10^{5}\end{array}\right]\right)$, the fol- 
lowing gain matrix is achieved:

$$
\begin{aligned}
\mathbf{K} & =\left[\begin{array}{cccccccc}
24523.2 & 3513.9 & 0 & 0 & -2572.2 & -361.4 & -3511.4 & -983.0 \\
24523.2 & 3513.9 & -2572.2 & -361.4 & 0 & 0 & 3511.4 & 983.0 \\
24523.2 & 3513.9 & 0 & 0 & 2572.2 & 361.4 & -3511.4 & -983.0 \\
24523.2 & 3513.9 & 2572.2 & 361.4 & 0 & 0 & 3511.4 & 983.0
\end{array}\right] \\
\mathbf{K}_{I} & =\left[\begin{array}{lccc}
50000.0 & 0 & -2236.1 & -5000 \\
50000.0 & -2236.1 & 0 & 5000 \\
50000.0 & 0 & 2236.1 & -5000 \\
50000.0 & 2236.1 & 0 & 5000
\end{array}\right] \\
\mathbf{K}_{\text {aug }} & =\left[\begin{array}{lll}
\mathbf{K} & -\mathbf{K}_{I}
\end{array}\right] .
\end{aligned}
$$

For the sake of simplicity, it was chosen to use a matrix $\mathbf{G}$ such that it considers uncoupled manifolds for each variable. Thus, $\lambda$ was chosen as equal to 0.1 and $\mathbf{G}$ as

$$
\mathbf{G}=\left[\begin{array}{cccccccccccc}
2 \lambda & 1 & 0 & 0 & 0 & 0 & 0 & 0 & \lambda^{2} & 0 & 0 & 0 \\
0 & 0 & 2 \lambda & 1 & 0 & 0 & 0 & 0 & 0 & \lambda^{2} & 0 & 0 \\
0 & 0 & 0 & 0 & 2 \lambda & 1 & 0 & 0 & 0 & 0 & \lambda^{2} & 0 \\
0 & 0 & 0 & 0 & 0 & 0 & 2 \lambda & 1 & 0 & 0 & 0 & \lambda^{2}
\end{array}\right]
$$

Figure 30 shows simulation and practical results from the application of this controller to the quadcopter. As happened to the other controllers, the prototype was able to track the reference but large oscillations were found in all three angles. The initial sliding surface value seen in Figure $30 \mathrm{f}$ is caused by the initial altitude the quadcopter was released. Although noisy, all the sliding surfaces stay close to zero, a necessary condition for this controller. 
Figure 30: Simulated and real results with SMC and LQR augmented with integrators.

(a) Simulated controlled states.

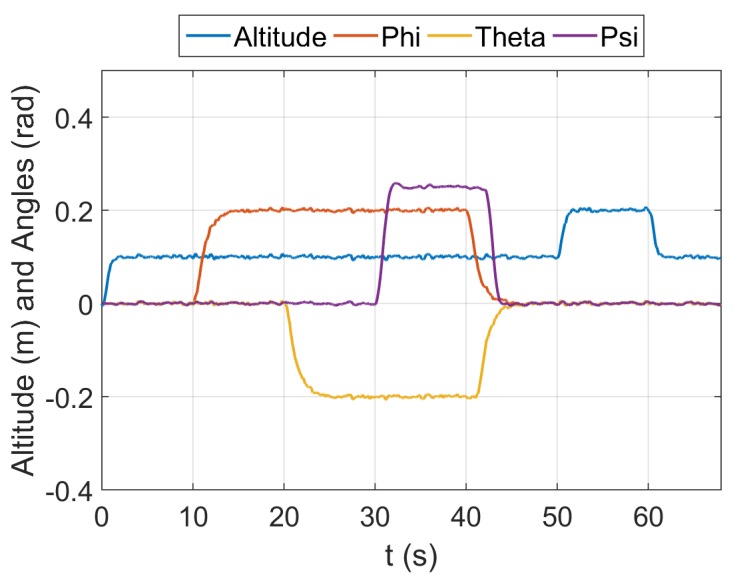

(c) Simulated control effort.

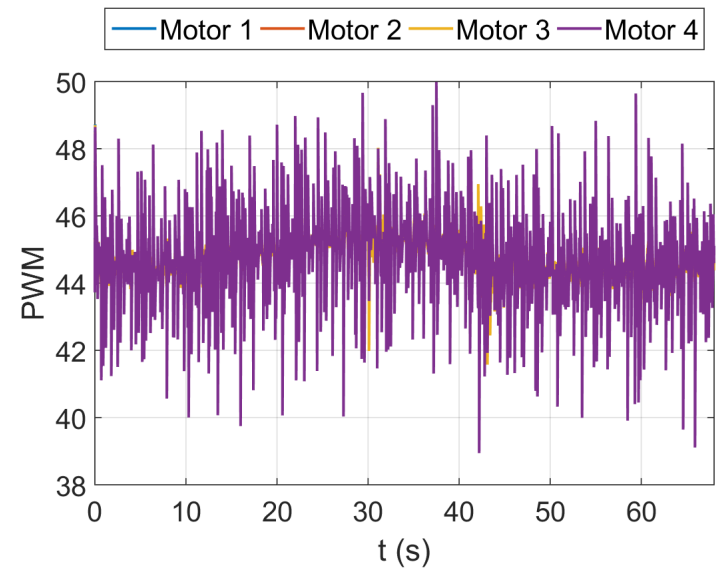

(e) Simulated sliding surfaces.

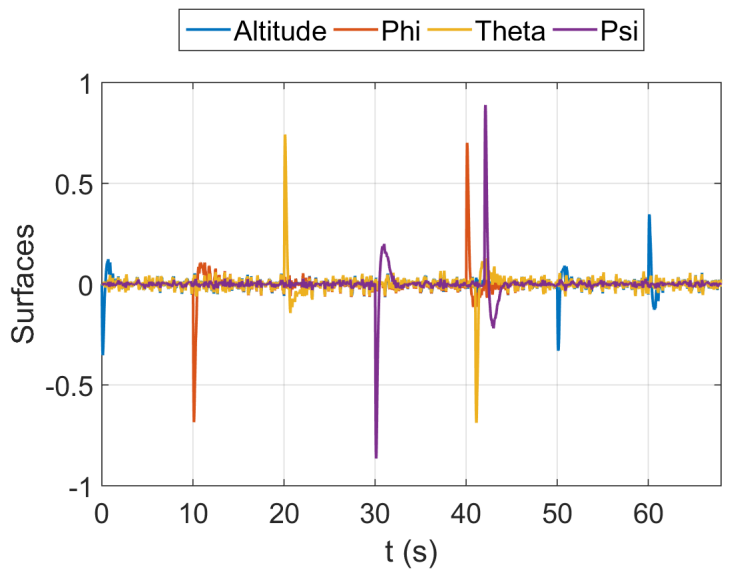

(b) Applied controlled states.

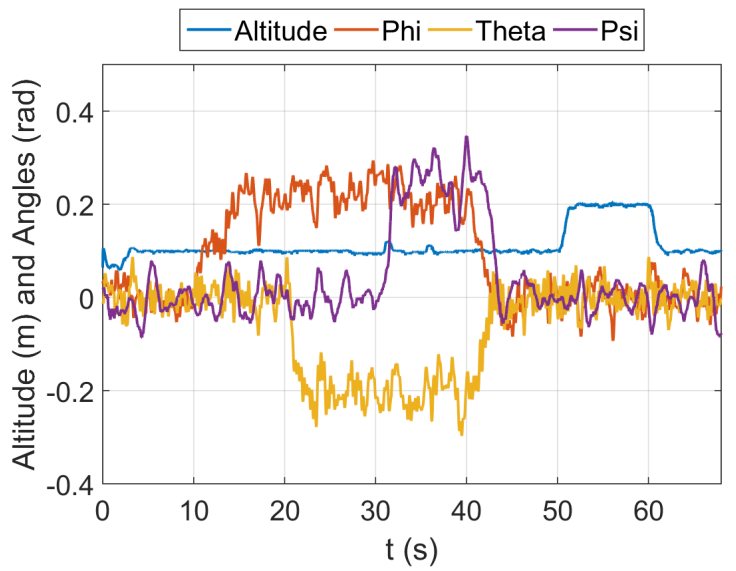

(d) Applied control effort.

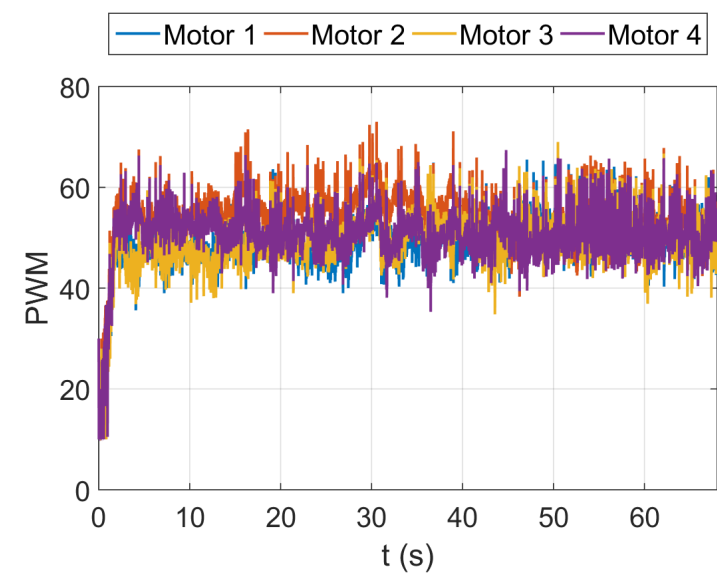

(f) Applied sliding surfaces.

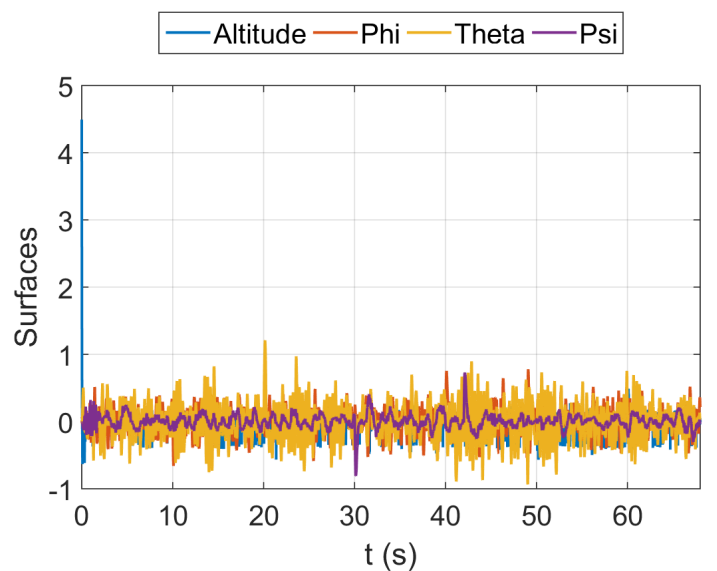

Source: author 


\subsection{Resultant Performance Indices}

Tables 6 and 7 present the performance indices found for the practical results. In the Controller column, each abbreviation stands for:

- PI+D: output-derivative PID;

- PI Dfree: derivative-free PID;

- LQR: linear quadratic regulator augmented with integrators;

- ISMCLQR: integral sliding mode control with LQR augmented with integrators.

Table 6: Angular Resultant Performance Indices

\begin{tabular}{l|ccc|ccc|ccc} 
& \multicolumn{3}{|c|}{ Phi } & \multicolumn{3}{|c|}{ Theta } & \multicolumn{3}{c}{ Psi } \\
Controller & IAE & ITAE & Var & IAE & ITAE & Var & IAE & ITAE & Var \\
\hline PI+D & 2.58 & 85.22 & 0.0025 & 2.46 & 84.69 & 0.0022 & 1.63 & 57.49 & 0.0011 \\
PI Dfree & 2.40 & 77.34 & 0.0022 & 2.24 & 74.14 & 0.0020 & 1.80 & 58.87 & 0.0014 \\
LQR & 2.05 & 78.39 & 0.0013 & 2.15 & 84.12 & 0.0014 & 1.55 & 63.34 & 0.0012 \\
ISMCLQR & 2.41 & 79.49 & 0.0023 & 1.98 & 66.90 & 0.0018 & 2.35 & 81.43 & 0.0028 \\
\hline
\end{tabular}

Table 7: Altitude Resultant Performance Indices

\begin{tabular}{lccc} 
Controller & IAE & ITAE & Var \\
\hline PI+D & 5.89 & 185.40 & 0.0015 \\
PI Dfree & 5.82 & 183.55 & 0.0015 \\
LQR & 7.09 & 268.93 & 0.0019 \\
ISMCLQR & 6.12 & 202.28 & 0.0016 \\
\hline
\end{tabular}

Altitude is, by far, the degree-of-freedom that accumulated more error, and a simple explanation for this is the fact that when pitch and roll are different than zero it makes it harder to the shaft to move, thus the altitude controller is not able to freely track the reference. Besides this, altitude presents the best overall result in the variance of the error. The angle $\psi$ presents better results than the others for every controller implemented but for the last.

Comparing the overall results by controller, LQR is the one that presented the best results for the angular motion while both PID implementations showed the best results for the altitude motion. 


\subsection{Trajectory Tracking}

The $X Y$ planar motion of an UAV can be controlled with a hierarchical scheme, in which the trajectory controller provides the desired angles to the angular controllers. It is important to highlight that guaranteeing that the angle controller is faster than the planar one is necessary.

An usual form of simplifying the planar equations of motion is assuming that $\phi$ and $\theta$ will be kept small and that $\psi$ will always be zero, i.e., the vehicle's heading will not change. The resulting equations are

$$
\ddot{X}=\theta \frac{U_{1}}{m} \text { and } \quad \ddot{Y}=-\phi \frac{U_{1}}{m},
$$

in which $U_{1}$ comes from the altitude controller and, for designing purposes, it is assumed to be hovering.

In the hierarchical controller described in this work, the planar controller generates desired pitch and roll angles depending on the position error, while the angular controllers provide the control inputs to the motors. However, the yaw angle is limited to stay close to zero due to the approximations assumed, thus the quadcopter would always point to the same direction independently of the route.

A simple way around this problem is to rotate the output of the planar controller (desired pitch and roll angles) with the following rotation matrix:

$$
\mathbf{R}=\left[\begin{array}{ccc}
\cos (-\psi) & -\sin (-\psi) & 0 \\
\sin (-\psi) & \cos (-\psi) & 0 \\
0 & 0 & 1
\end{array}\right]
$$

Therefore, the planar controller is simply setting generic angles of reference for the system, which are corrected by the rotation to serve as inputs for the pitch and roll controllers.

To test the effectiveness of the proposed controllers, it was implemented a point-ofinterest trajectory, in which the vehicle has to track a circular path while pointing to its center. Defining the circumference center at the origin of the $X Y$ system, the reference signal to the planar controller is given by

$$
\begin{aligned}
& X_{r}(t)=r \cos (\omega t) \\
& Y_{r}(t)=r \sin (\omega t),
\end{aligned}
$$


with $r$ being the radius and $\omega$ the frequency. Yaw angle reference signal can be generated based on the vehicles position:

$$
\psi_{r}(t)=\operatorname{atan} 2(Y, X)+2 k \pi+\pi,
$$

with $k$ counting the number of times the atan 2 function passed through its discontinuity.

The lower level controller is the same as presented in Section 4.7, while the planar motion is controller by LQR augmented with integrators. Figure 31 presents simulation results for this implementation, in which is desired to follow a circular path of $5 \mathrm{~m}$ radius and $0.2 \mathrm{rad} / \mathrm{s}$ frequency. Figure 31a depicts the trajectory divided into $X Y Z$ components; Figures 31b and 31c present each angle reference and Figure 31d shows the motors references. Lastly, Figure 32 presents the $3 \mathrm{D}$ view of the trajectory.

Moving to the simulation results obtained for the circular reference tracking, $X Y$ position outputs are $3 \mathrm{~s}$ delayed from reference and $2 \%$ damped. The initial oscillations either in $\phi$ and $\theta$ angles (Figure 31b) and in motors references (Figure 31d) are caused by the difference between initial states and reference signal. 
Figure 31: Reference tracking simulation results.

(a) $X Y Z$ movement.

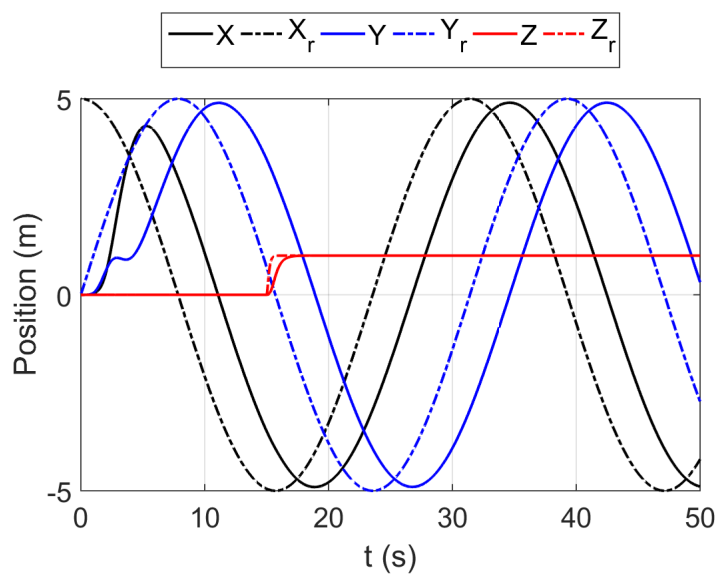

(c) Angle $\psi$.

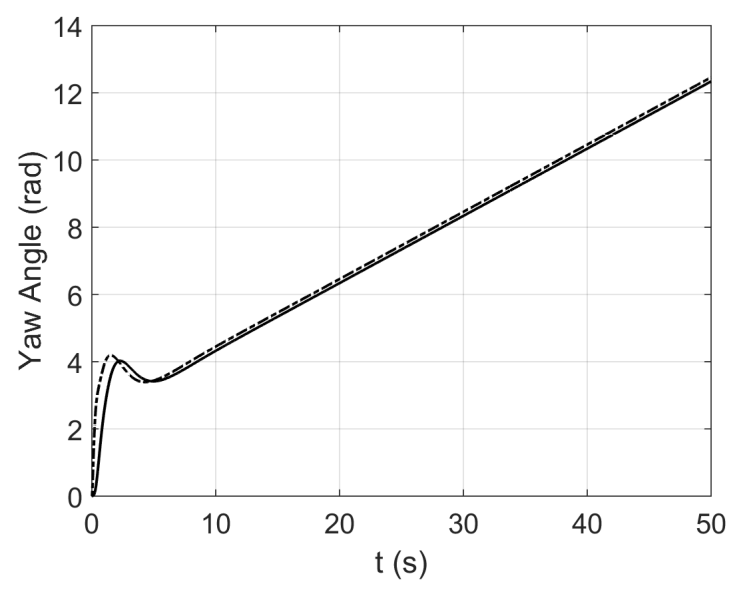

(b) Angles $\phi$ and $\theta$.

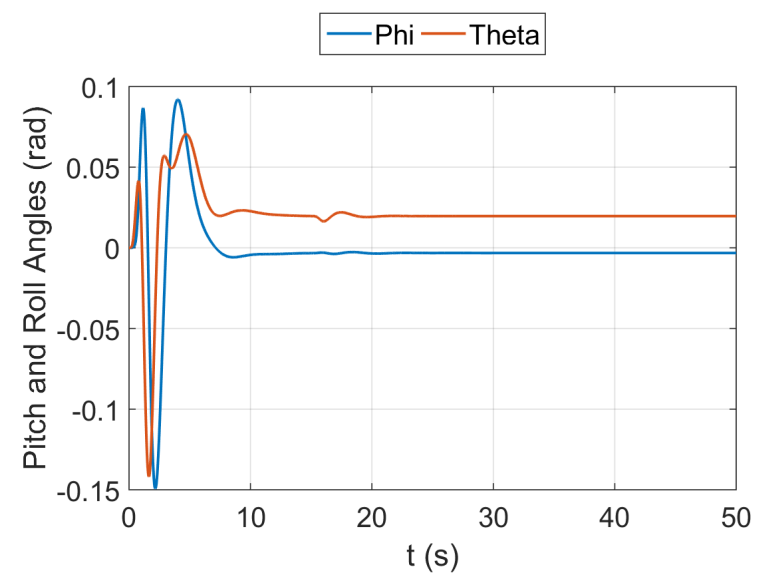

(d) Motors references.

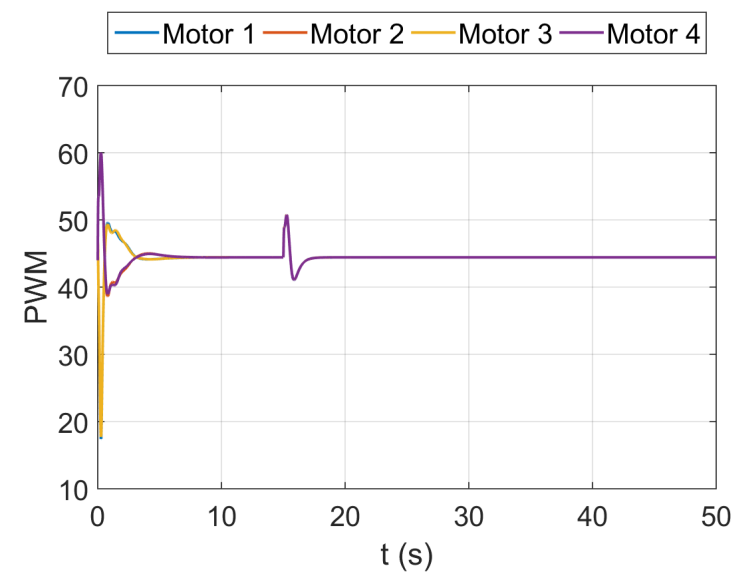

Source: author

Figure 32: Reference tracking view in 3D.

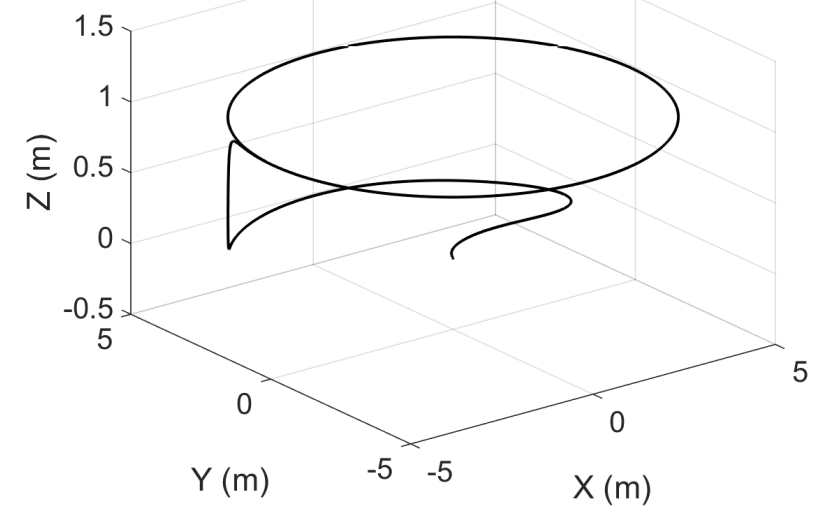

Source: author 


\section{CONCLUSION}

Unmanned Aerial Vehicles have been trending on research themes for years and will be for many more. They can be used in studies that vary from simple linear attitude controllers to trajectory planning and tracking and from high-speed maneuvers capability to self-learning algorithms. The market already offers a wide variety of products and applications, but there is still much to be researched.

The four-degree-of-freedom quadcopter prototype proposed on this work uses the same parts as commercial ones except for the battery, which was replaced by a $12 \mathrm{~V} 500 \mathrm{~W}$ power supply. Brushless DC motors, 5-inch propellers and 12A ESCs have been tested and their characteristics identified.

The propeller guards made a great protection against accidents, yet remaining lightweight and not blocking the airflow. The ball head used has very low friction and allowed close-to-real movement around the axes. Although linear bearings were used, it still provided reasonable resistance in the altitude axis due to shaft imperfections; besides, it is also known that they were placed too close to each other, allowing the shaft to tilt inside them, causing $X Y$ displacements, aggravated by the elasticity of the shaft in these directions; this is thought to be the main cause of the oscillations in all previous results.

Even though FreeIMU is intended to be an easy-to-use tool, it demands some great time understanding how it works, how to correctly set it up and to calibrate it. In the sensor fusion, a proportional and an integral gain have to be tuned in order to guarantee convergence and a good trade-off: setting a high proportional gain makes it noisier, a low one lets it slower; a high integral gain slows convergence, while a steady-state error appears with a low one.

Four controllers were designed, simulated and applied: PID, LQR, Sliding Mode and a combination of Sliding Mode with LQR. The first had two implementations, one that takes the derivative from the output and not from the error between it and the reference, and other that takes advantage of the speed estimative given by the sensors and does not 
derive the output signal, reason for being called "derivative-free".

The Linear Quadratic Regulator had integrators inserted to each channel to allow the system to track a trajectory. The speed estimative given by the sensors were also used in this controller, as it is necessary to feedback all the states.

The usual Sliding Mode Controller is the only one tested that was not able to stabilize the prototype. This happened due to the fact that it presents good robustness guarantees to mass and inertia variation, but not to motor and propeller parameters.

This work also proposed a new type of controller: using Sliding Modes alongside LQR augmented with integrators. This method takes advantage of the tracking capabilities of the latter and robustness of the former. The main benefits are the guarantee of null steady-state error for step inputs and the requirement of the first derivative of the reference only; none of these are achieved with usual SMC methods.

Ultimately, the method presented for planar tracking overcame the yaw angle limitation, brought by linearization, allowing the quadcopter to receive position and direction references. Moreover, the hierarchical scheme was also effective in controlling the UAV in two steps, in which the position controller generates reference angles, achieved by the angular controller.

Lastly, admitting the system to be decoupled showed to be a valid assumption since the coupling effects are almost not visible in the simulation figures presented in the text. Altitude is the most sensible state because it depends directly on pitch and roll angles, but all designed controllers were able to handle it.

Future works will make use of the developed prototype but with a new microcontroller. Aiming at getting closer to the most used softwares for robots, it is desired to use ROS (QUIGLEY et al., 2009), the Robot Operating System, widely used in universities and industries. ROSflight (JACKSON; ELLINGSON; MCLAIN, 2016) is an autopilot system designed for integration with ROS, a solution that connects a personal computer running Linux to an onboard Flip32 flight controller. 


\section{REFERENCES}

ARGENTIM, L. M. et al. PID, LQR and LQR-PID on a quadcopter platform. In: International Conference on Informatics, Electronics Vision (ICIEV). [S.l.: s.n.], 2013. p. $1-6$.

BEFINS, K. Localization and System Identification of a Quadcopter UAV. Thesis (Masters) - Dept. Mechanical and Aeronautical Eng., Western Michigan Univ, Kalamazoo, MI, USA, 2014.

BOUABDALLAH, S. Design and control of quadrotors with application to autonomous flying. Thesis $(\mathrm{PhD})$ - École Polytechnique Fédérale de Lausanne, Lausanne, France, 2007.

BOUSBAINE, A.; WU, M. H.; POYI, G. T. Modelling and simulation of a quad-rotor helicopter. In: 6th IET International Conference on Power Electronics, Machines and Drives (PEMD 2012). [S.l.: s.n.], 2012. p. 1-6.

BRESCIANI, T. Modelling, Identification and Control of a Quadrotor Helicopter. Thesis (Masters) - Department of Automatic Control, Lund University, Lund, Sweden, 2008.

CARIñO, J.; ABAUNZA, H.; CASTILLO, P. Quadrotor quaternion control. In: International Conference on Unmanned Aircraft Systems (ICUAS). [S.l.: s.n.], 2015. p. $825-831$.

CASTANOS, F.; FRIDMAN, L. Analysis and design of integral sliding manifolds for systems with unmatched perturbations. IEEE Transactions on Automatic Control, v. 51, n. 5, p. 853-858, May 2006.

CASTRUCCI, P. de L.; BITTAR, A.; SALES, R. M. Controle Automático. [S.l.]: LTC, 2011.

EUSTON, M. et al. A complementary filter for attitude estimation of a fixed-wing UAV. In: 2008 IEEE/RSJ International Conference on Intelligent Robots and Systems. [S.l.: s.n.], 2008. p. $340-345$.

FADALI, M. S.; VISIOLI, A. Digital Control Engineering. 2th. ed. [S.l.]: Elsevier, 2012.

FIGUEROA, R. et al. Reinforcement learning for balancing a flying inverted pendulum. In: 11th World Congress on Intelligent Control and Automation (WCICA). [S.l.: s.n.], 2014. p. 1787-1793.

FRESK, E.; NIKOLAKOPOULOS, G. Full quaternion based attitude control for a quadrotor. In: European Control Conference (ECC). [S.1.: s.n.], 2013. p. 3864-3869.

GEROMEL, J. C.; KOROGUI, R. H. Controle Linear de Sistemas Dinâmicos: Teoria, Ensaios Práticos e Exercícios. [S.1.]: Blucher, 2011. 
HAMAYUN, M. T.; EDWARDS, C.; ALWI, H. Fault Tolerant Control Schemes Using Integral Sliding Modes. [S.l.]: Springer International Publishing, 2016.

HEHN, M.; D'ANDREA, R. A flying inverted pendulum. In: IEEE International Conference on Robotics and Automation (ICRA). [S.l.: s.n.], 2011. p. 763-770.

HEHN, M.; D'ANDREA, R. Quadrocopter trajectory generation and control. IFAC Proceedings Volumes, Elsevier, v. 44, n. 1, p. 1485-1491, 2011.

HEHN, M.; D'ANDREA, R. A frequency domain iterative learning algorithm for high-performance, periodic quadrocopter maneuvers. Mechatronics, Elsevier, v. 24, n. 8, p. 954-965, 2014.

HONEYWELL. 3-Axis Digital Compass IC HMC5883L. [S.l.], 2013. Rev E.

HUYNH, M. Q.; ZHAO, W.; XIE, L. L1 adaptive control for quadcopter: Design and implementation. In: 13th International Conference on Control Automation Robotics Vision (ICARCV). [S.l.: s.n.], 2014. p. 1496-1501.

HYSTAD, A. V. Model, Design and Control of a Quadcopter. Thesis (Masters) Department of Engineering Cybernetics, NTNU, Trondheim, Norway, 2015.

INVENSENSE INC. MPU-6000 and MPU-6050 Product Specification. [S.l.], 2013. Revision 3.4.

JACKSON, J.; ELLINGSON, G.; MCLAIN, T. ROSflight: A lightweight, inexpensive MAV research and development tool. In: 2016 International Conference on Unmanned Aircraft Systems (ICUAS). [S.l.: s.n.], 2016. p. 758-762.

LEONG, B. T. M.; LOW, S. M.; OOI, M. P.-L. Low-cost microcontroller-based hover control design of a quadcopter. Procedia Engineering, Elsevier, v. 41, p. 458 - 464, 2012. ISSN 1877-7058.

LUPASHIN, S.; D'ANDREA, R. Adaptive open-loop aerobatic maneuvers for quadrocopters. $\{$ IFAC $\}$ Proceedings Volumes, v. 44, n. 1, p. $2600-2606,2011$. 18th $\{$ IFAC $\}$ World Congress.

LUPASHIN, S. et al. A platform for aerial robotics research and demonstration: The flying machine arena. Mechatronics, Elsevier, v. 24, n. 1, p. 41 - 54, 2014.

LUUKKONEN, T. Modelling and control of quadcopter. Independent research project in applied mathematics, Espoo, 2011.

MADGWICK, S. O.; HARRISON, A. J.; VAIDYANATHAN, R. Estimation of IMU and MARG orientation using a gradient descent algorithm. In: IEEE. 2011 IEEE International Conference on Rehabilitation Robotics. [S.l.], 2011. p. 1-7.

MAHONY, R.; HAMEL, T.; PFLIMLIN, J. M. Nonlinear complementary filters on the special orthogonal group. IEEE Transactions on Automatic Control, v. 53, n. 5, p. 1203-1218, June 2008.

MAHONY, R.; KUMAR, V.; CORKE, P. Multirotor aerial vehicles: Modeling, estimation, and control of quadrotor. IEEE Robotics Automation Magazine, v. 19, n. 3, p. 20-32, Sept 2012. 
MARTINEZ, V. M. Modelling of the Flight Dynamics of a Quadrotor Helicopter. Thesis (Masters) - Dept. of Aerospace Sciences, Cranfield Univ, Cranfield, UK, 2007.

MEASUREMENT SPECIALTIES. MS5611-01BA03 Barometric Pressure Sensor, with stainless steel cap. [S.l.], 2012.

MIAN, A. A.; DAOBO, W. Modeling and backstepping-based nonlinear control strategy for a 6 DOF quadrotor helicopter. Chinese Journal of Aeronautics, v. 21, n. 3, p. 261 268, 2008. ISSN 1000-9361.

MICHAEL, N. et al. The GRASP multiple micro-UAV testbed. IEEE Robotics Automation Magazine, v. 17, n. 3, p. 56-65, Sept 2010.

MUELLER, M. W.; D'ANDREA, R. Stability and control of a quadrocopter despite the complete loss of one, two, or three propellers. In: 2014 IEEE International Conference on Robotics and Automation (ICRA). [S.l.: s.n.], 2014. p. 45-52.

MUELLER, M. W.; HAMER, M.; D'ANDREA, R. Fusing ultra-wideband range measurements with accelerometers and rate gyroscopes for quadrocopter state estimation. In: 2015 IEEE International Conference on Robotics and Automation (ICRA). [S.l.: s.n.], 2015. p. 1730-1736.

MUSTAPA, Z. et al. Altitude controller design for multi-copter UAV. In: International Conference on Computer, Communications, and Control Technology (I4CT). [S.l.: s.n.], 2014. p. 382-387.

NEMATI, A.; KUMAR, M. Modeling and control of a single axis tilting quadcopter. In: 2014 American Control Conference. [S.l.: s.n.], 2014. p. 3077-3082. ISSN 0743-1619.

NICE, E. Design of a four rotor hovering vehicle. Thesis (Masters) - Cornell University, Ithaca, NY, USA, 2004.

NICOL, C.; MACNAB, C.; RAMIREZ-SERRANO, A. Robust adaptive control of a quadrotor helicopter. Mechatronics, v. 21, n. 6, p. 927 - 938, 2011.

OGATA, K. Engenharia de Controle Moderno. [S.1.]: Pearson, 2003.

OLIVEIRA, M. D. Modeling, Identification and Control of a Quadrotor Aircraft. Thesis (Masters) - Dept. of Control Eng., Czech Technical Univ, Prague, Czech Rep., 2011.

PFEIFER, E. Projeto e Controle de um UAV Quadrirotor. Thesis (Masters) Engenharia de Sistemas, Escola Politécnica da USP, São Paulo, Brasil, 2013.

QUIGLEY, M. et al. ROS: an open-source robot operating system. In: ICRA workshop on open source software. [S.l.: s.n.], 2009. v. 3, p. 5.

RAFFO, G. V.; ORTEGA, M. G.; RUBIO, F. R. An integral predictive/nonlinear $\mathrm{H}_{\infty}$ control structure for a quadrotor helicopter. Automatica, v. 46, n. 1, p. 29 - 39, 2010. ISSN 0005-1098.

RITZ, R.; D'ANDREA, R. An on-board learning scheme for open-loop quadrocopter maneuvers using inertial sensors and control inputs from an external pilot. In: 2014 IEEE International Conference on Robotics and Automation (ICRA). [S.l.: s.n.], 2014. p. $5245-5251$. 
SA, I.; CORKE, P. Estimation and control for an open-source quadcopter. In: Australasian Conference on Robotics and Automation (ACRA 2011). Monash University, Melbourne, Vic: [s.n.], 2011.

SANCA, A. Modelagem e controle de um microveículo aéreo: uma aplicação de estabilidade robusta com a técnica backstepping em uma estrutura hexarrotor. Thesis (PhD) — Universidade Federal do Rio Grande do Norte, Natal, 2013.

SLOTINE, J.-J. E.; LI, W. Applied Nonlinear Control. [S.l.]: Pearson, 1991.

SUMANTRI, B. et al. Robust tracking control of a quad-rotor helicopter utilizing sliding mode control with a nonlinear sliding surface. Journal of System Design and Dynamics, v. 7, n. 2, p. 226-241, 2013.

TAYEBI, A.; MCGILVRAY, S. Attitude stabilization of a VTOL quadrotor aircraft. IEEE Transactions on Control Systems Technology, v. 14, n. 3, p. 562-571, May 2006.

VALENTI, M. et al. Indoor multi-vehicle flight testbed for fault detection, isolation, and recovery. In: Proceedings of the AIAA Guidance, Navigation, and Control Conference and Exhibit, Keystone, CO. [S.l.: s.n.], 2006. v. 63, p. 64.

VARESANO, F. FreeIMU: An open hardware framework for orientation and motion sensing. CoRR, abs/1303.4949, 2013. Disponível em: 〈http://arxiv.org/abs/1303.4949〉.

XIONG, J.-J.; ZHENG, E.-H. Position and attitude tracking control for a quadrotor UAV. ISA transactions, Elsevier, v. 53, n. 3, p. 725-731, 2014.

YU, Y.; DING, X. A quadrotor test bench for six degree of freedom flight. Journal of Intelligent \& Robotic Systems, v. 68, n. 3, p. 323-338, 2012.

ZHENG, E.-H.; XIONG, J.-J.; LUO, J.-L. Second order sliding mode control for a quadrotor UAV. ISA transactions, Elsevier, v. 53, n. 4, p. 1350-1356, 2014. 


\section{APPENDIX A - EXTRA RESULTS}

This appendix contains preliminary results for the controllers presented in the text.

\section{A.1 PID}

Output derivative and derivative-free PID implementations results were presented in the body of this text, but not the ISA form. Figure 33a and 33b show the controlled states with this controller and its control effort, while Figures $33 \mathrm{c}$ and $33 \mathrm{~d}$ were generated considering measurement noise.

\section{A.2 Sliding Mode}

Four Sliding Mode Controllers have been designed: one for altitude and one for each of the three angles. In order to prove the performance of each, initial conditions different from zero were set to them separately, and their response, surface $s$ and control effort were plotted. In the surface plots, the black-dash-dotted lines represent the layer thickness $\Phi$ of each controller.

Figure 34 presents a noise-free simulation of this controller while Figures 35 and 36 display the controllers responses to non-zero initial conditions. More specifically, Figure 35a shows the quadcopter altitude for an initial condition hovering at $-1 \mathrm{~m}$, its surface is plotted in Figure 35c and the control effort is in Figure 35e. Each angle were subject to the same initial condition of $1 \mathrm{rad}$, with $\phi, \theta$ and $\psi$ responses being presented in Figures 35b, $36 \mathrm{a}$ and 36b, respectively, while the surfaces are in Figures 35d, 36c and 36d and the control efforts in in Figures 35f, 36e and 36f. 
Figure 33: Simulation results for the discretized PID controllers in ISA form.

(a) Controlled states in ISA form PID.

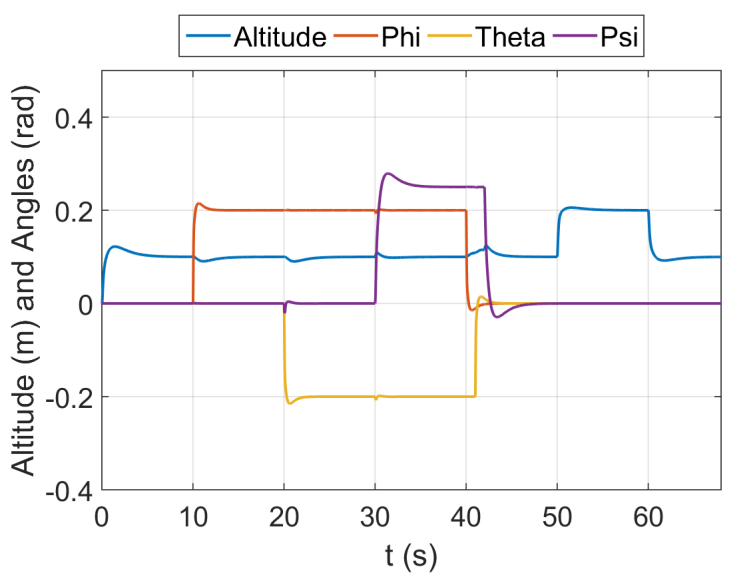

(c) Controlled states in ISA form PID with noise.

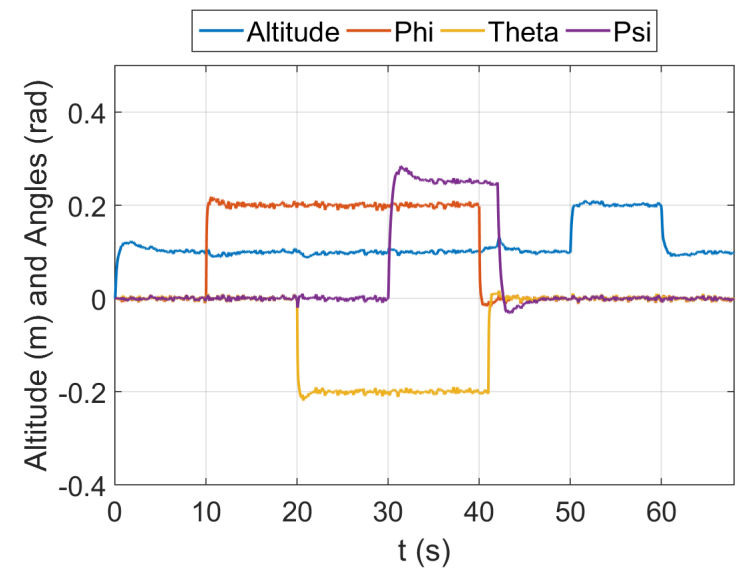

(b) Control effort in ISA form PID.

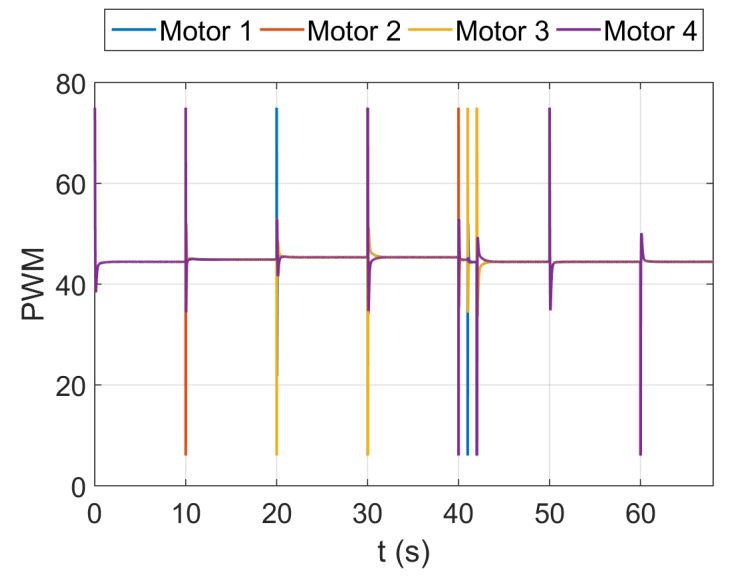

(d) Control effort in ISA form PID with noise.

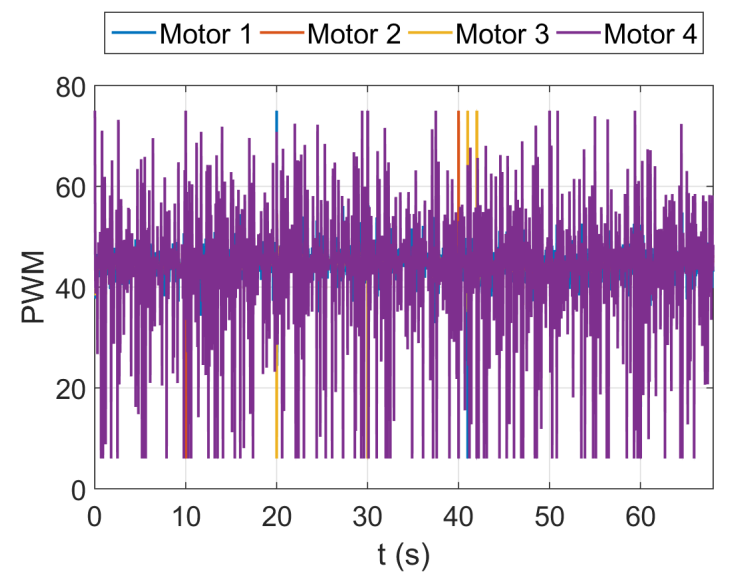

Source: author

Figure 34: Sliding Mode Controllers

(a) States without noise.

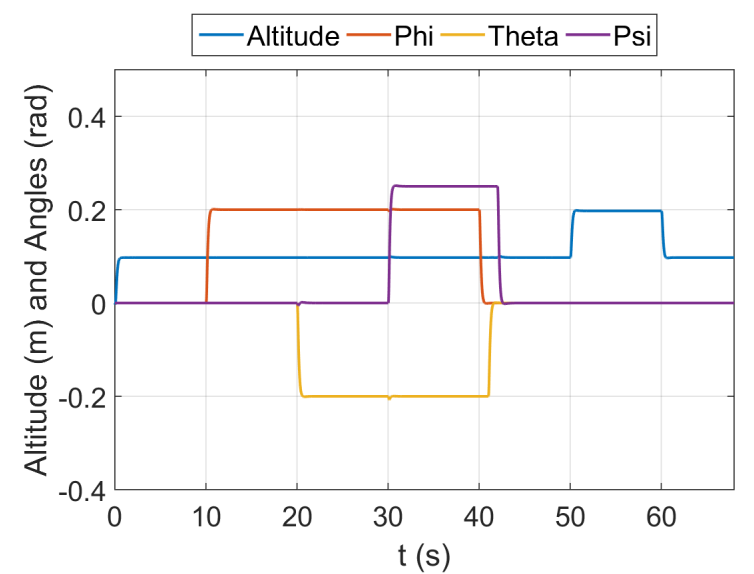

(b) Control effort without noise.

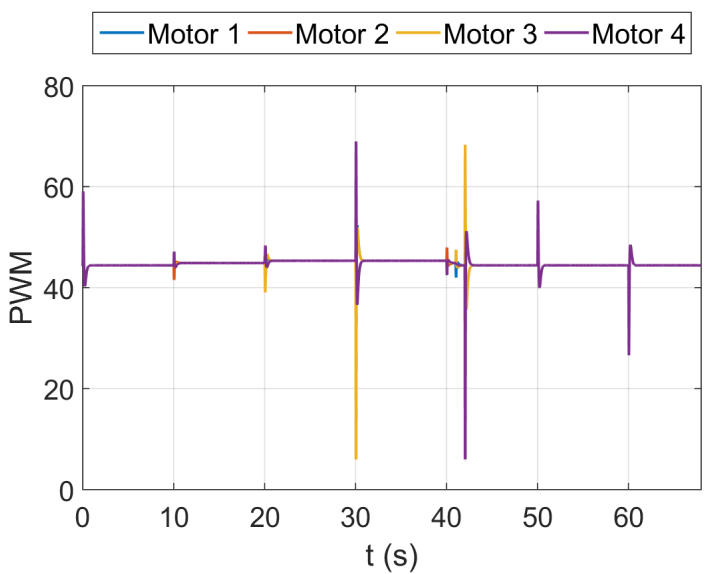

Source: author 
Figure 35: Altitude and $\phi$ response to non-zero initial condition with SMC.

(a) Altitude with initial condition at $-1 \mathrm{~m}$.

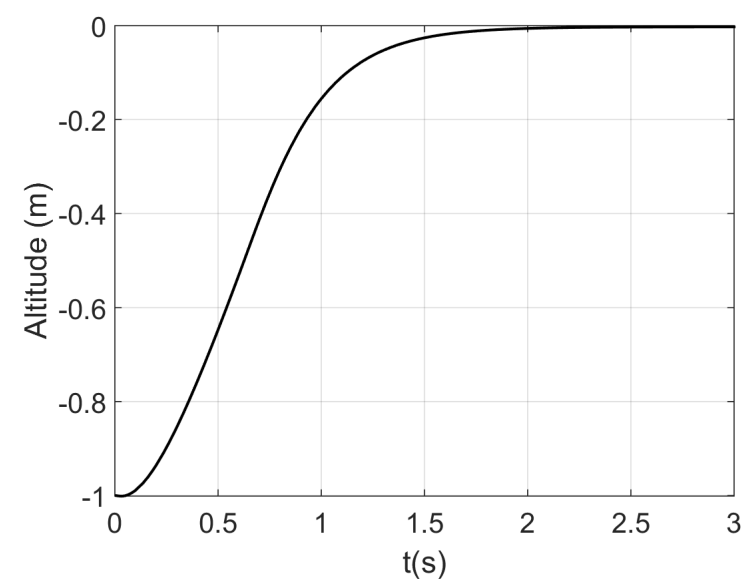

(c) Altitude control surface.

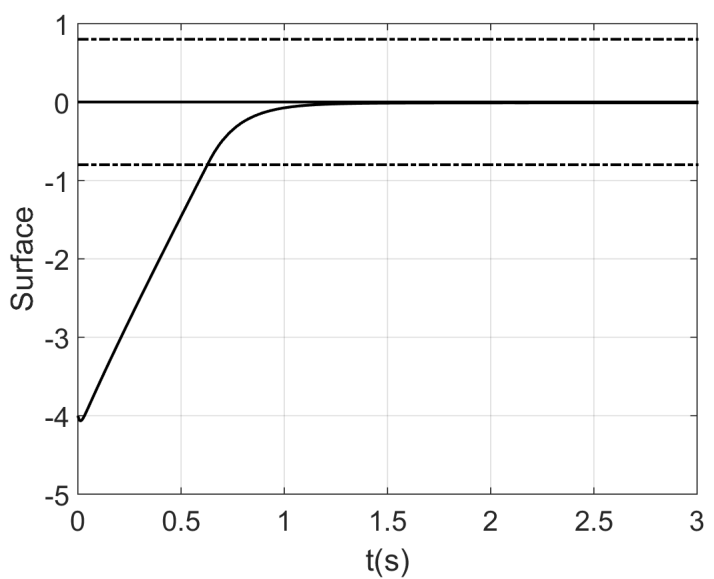

(e) Altitude control effort.

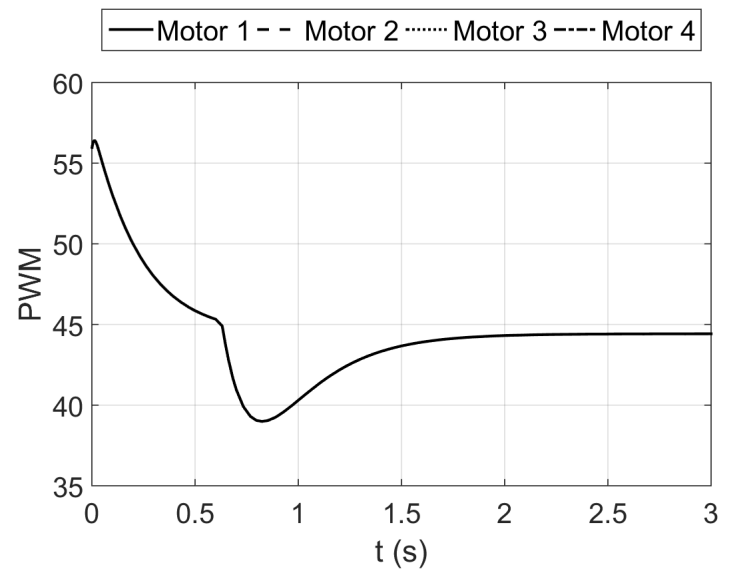

(b) $\phi$ with initial condition at 1rad.

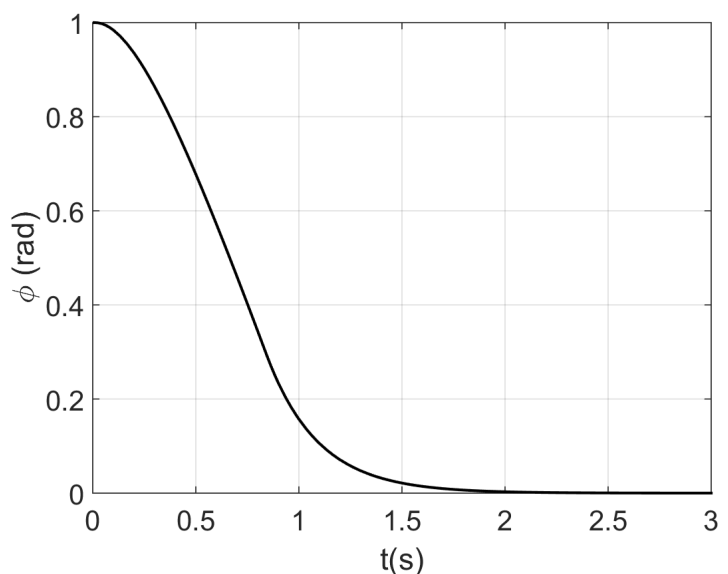

(d) $\phi$ control surface.

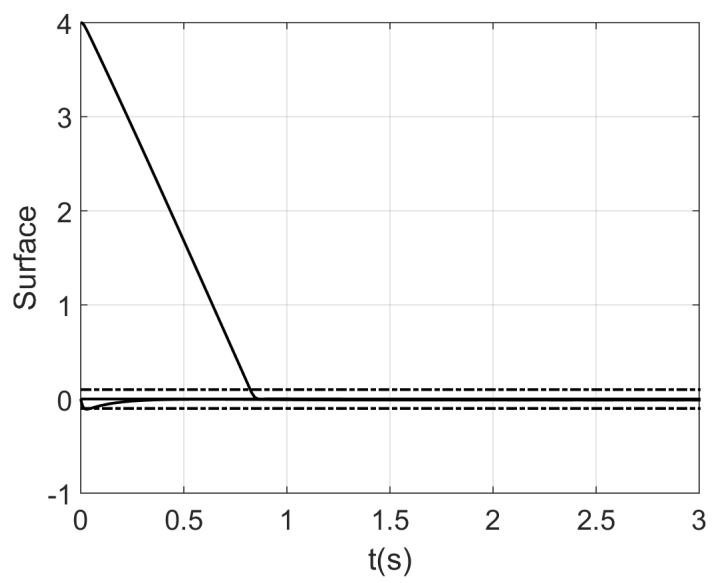

(f) $\phi$ control effort.

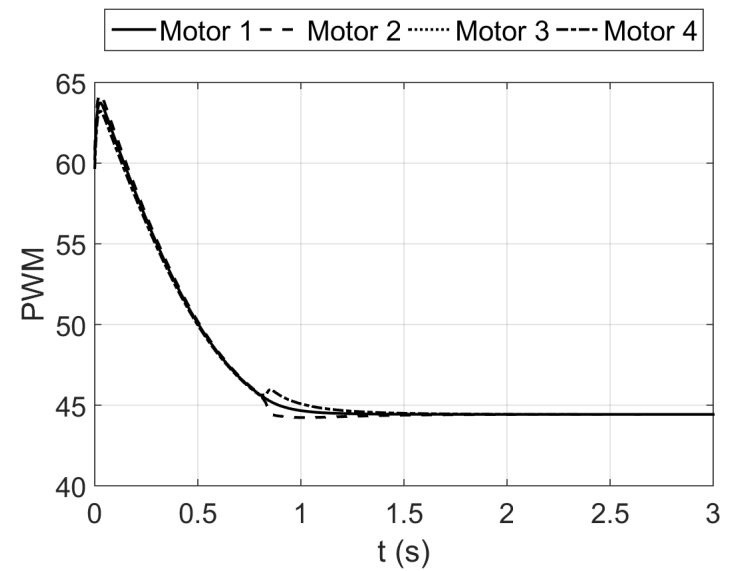

Source: author 
Figure 36: $\theta$ and $\psi$ response to non-zero initial condition with SMC.

(a) $\theta$ with initial condition at $1 \mathrm{rad}$.

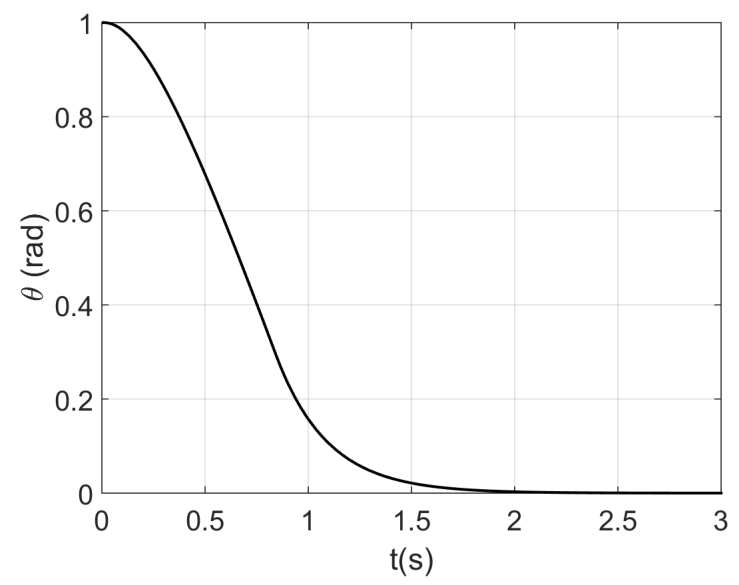

(c) $\theta$ control surface.

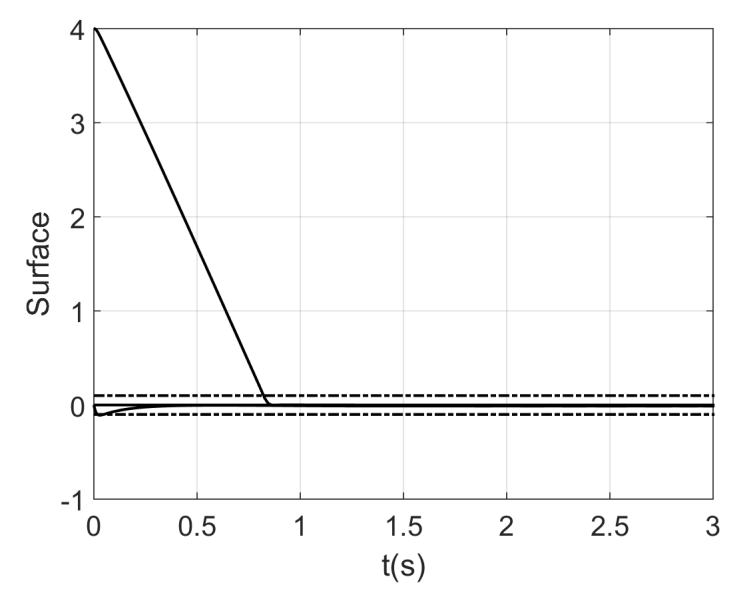

(e) $\theta$ control effort.

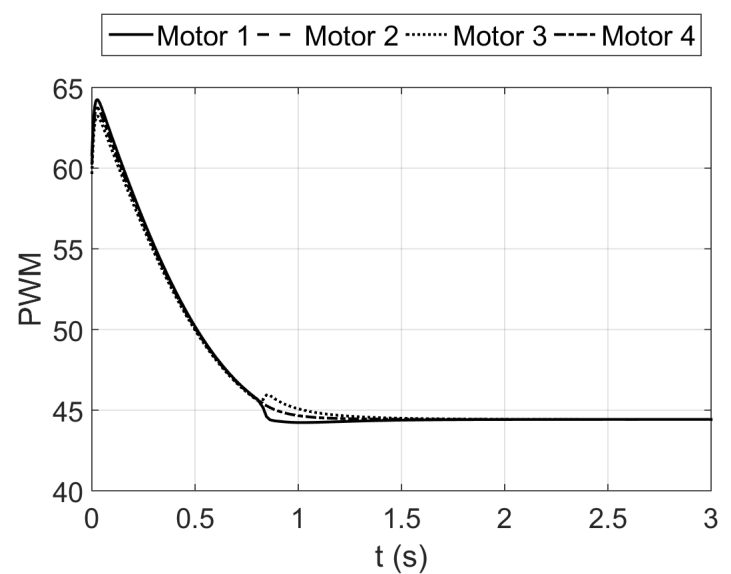

(b) $\psi$ with initial condition at 1rad.

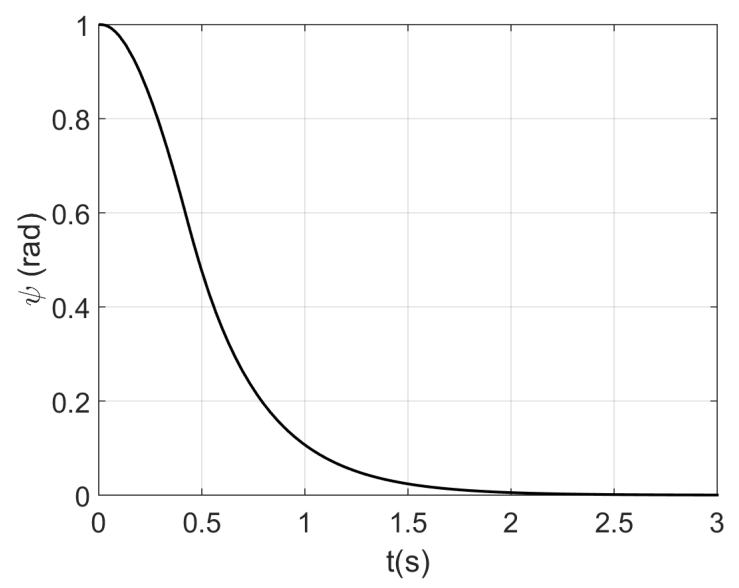

(d) $\psi$ control surface.

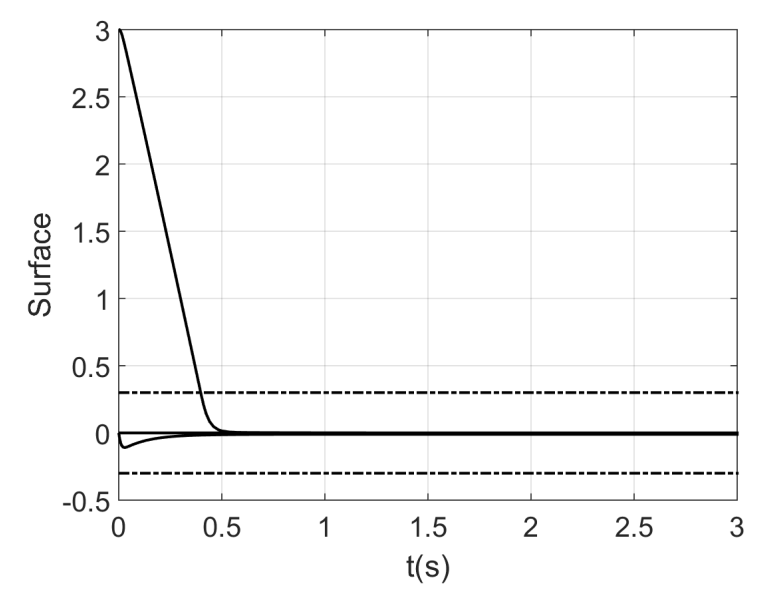

(f) $\psi$ control effort.

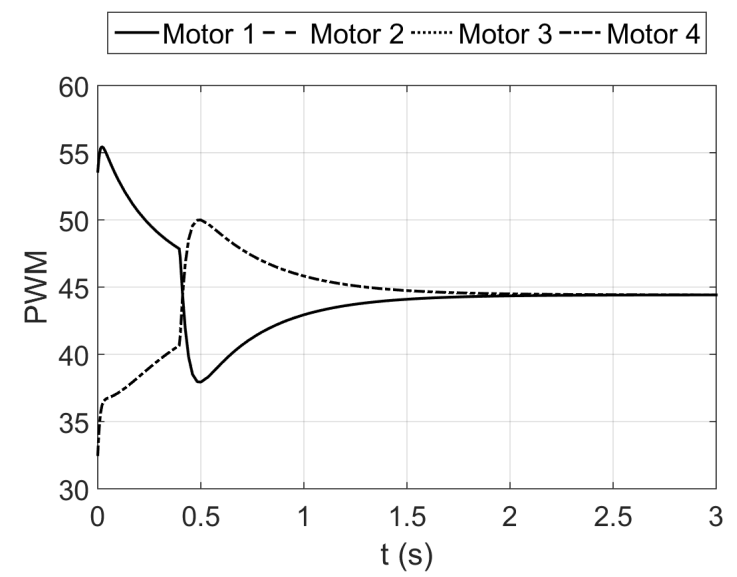

Source: author 